\title{
Search for neutral MSSM Higgs bosons decaying to a pair of tau leptons in pp collisions
}

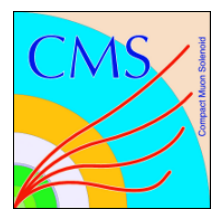

\section{The CMS collaboration}

\section{E-mail: cms-publication-committee-chair@cern.ch}

ABstRACT: A search for neutral Higgs bosons in the minimal supersymmetric extension of the standard model (MSSM) decaying to tau-lepton pairs in pp collisions is performed, using events recorded by the CMS experiment at the LHC. The dataset corresponds to an integrated luminosity of $24.6 \mathrm{fb}^{-1}$, with $4.9 \mathrm{fb}^{-1}$ at $7 \mathrm{TeV}$ and $19.7 \mathrm{fb}^{-1}$ at $8 \mathrm{TeV}$. To enhance the sensitivity to neutral MSSM Higgs bosons, the search includes the case where the Higgs boson is produced in association with a b-quark jet. No excess is observed in the tau-lepton-pair invariant mass spectrum. Exclusion limits are presented in the MSSM parameter space for different benchmark scenarios, $m_{\mathrm{h}}^{\max }, m_{\mathrm{h}}^{\bmod +}, m_{\mathrm{h}}^{\bmod -}$, light-stop, lightstau, $\tau$-phobic, and low- $m_{\mathrm{H}}$. Upper limits on the cross section times branching fraction for gluon fusion and b-quark associated Higgs boson production are also given.

KEYWORDS: Supersymmetry, Hadron-Hadron Scattering, Higgs physics

ARXIV EPRINT: 1408.3316 


\section{Contents}

1 Introduction 1

1.1 MSSM Higgs boson benchmark scenarios 2

2 Experimental setup, event reconstruction, and simulation 5

$\begin{array}{llr}3 & \text { Event selection } & 7\end{array}$

$\begin{array}{llr}4 & \text { Background estimation } & 8\end{array}$

5 Tau lepton-pair invariant mass $\quad 9$

6 Systematic uncertainties $\quad 11$

7 Statistical analysis $\quad 14$

$\begin{array}{lll}8 & \text { Results } & 17\end{array}$

$\begin{array}{lll}9 & \text { Summary } & 19\end{array}$

$\begin{array}{ll}\text { A Exclusion limits } & 23\end{array}$

$\begin{array}{ll}\text { The CMS collaboration } & 37\end{array}$

\section{Introduction}

A broad variety of precision measurements have shown the overwhelming success of the standard model (SM) [1-3] of fundamental interactions, which includes an explanation for the origin of the mass of the weak force carriers, as well as for the quark and lepton masses. In the SM, this is achieved via the Brout-Englert-Higgs mechanism [4-9], which predicts the existence of a scalar boson, the Higgs boson. However, the Higgs boson mass in the SM is not protected against quadratically divergent quantum-loop corrections at high energy, known as the hierarchy problem. In the model of supersymmetry (SUSY) [10, 11], which postulates a symmetry between the fundamental bosons and fermions, a cancellation of these divergences occurs naturally. The Higgs sector of the minimal supersymmetric extension of the standard model (MSSM) $[12,13]$ contains two scalar doublets that result in five physical Higgs bosons: a light and a heavy CP-even Higgs boson $\mathrm{h}$ and $\mathrm{H}$, a CP-odd Higgs boson A, and two charged Higgs bosons $\mathrm{H}^{ \pm}$. At tree level the Higgs sector can be expressed in terms of two parameters which are usually chosen as the mass of the CP-odd Higgs boson $m_{\mathrm{A}}$ and $\tan \beta$, the ratio of the two vacuum expectation values of the two Higgs doublets. 

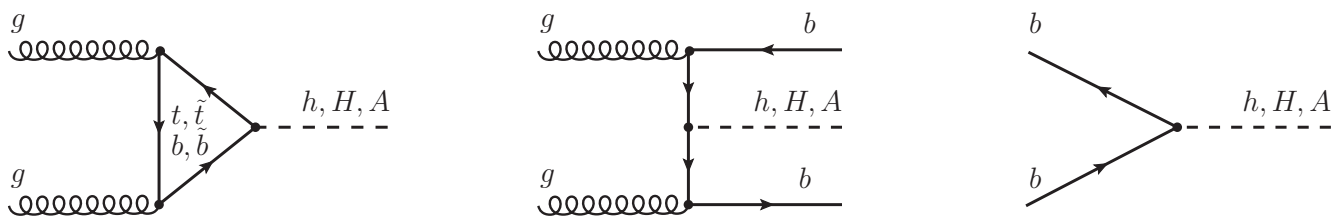

Figure 1. Leading-order diagrams of the gluon fusion (left) and b-quark associated Higgs boson production, in the four-flavor (center) and the five-flavor (right) scheme.

The dominant neutral MSSM Higgs boson production mechanism is the gluon fusion process for small and moderate values of $\tan \beta$. At large values of $\tan \beta$ b-quark associated production is the dominant contribution, due to the enhanced Higgs boson Yukawa coupling to $\mathrm{b}$ quarks. Figure 1 shows the leading-order diagrams for the gluon fusion and b-quark associated Higgs boson production, in the four-flavor and in the five-flavor scheme. In the region of large $\tan \beta$ the branching fraction to tau leptons is also enhanced, making the search for neutral MSSM Higgs bosons in the $\tau \tau$ final state particularly interesting.

This paper reports a search for neutral MSSM Higgs bosons in pp collisions at $\sqrt{s}=$ $7 \mathrm{TeV}$ and $8 \mathrm{TeV}$ in the $\tau \tau$ decay channel. The data were recorded with the CMS detector [14] at the CERN LHC and correspond to an integrated luminosity of $24.6 \mathrm{fb}^{-1}$, with $4.9 \mathrm{fb}^{-1}$ at $7 \mathrm{TeV}$ and $19.7 \mathrm{fb}^{-1}$ at $8 \mathrm{TeV}$. Five different $\tau \tau$ signatures are studied, $\mathrm{e} \tau_{\mathrm{h}}, \mu \tau_{\mathrm{h}}, \mathrm{e} \mu, \mu \mu$, and $\tau_{\mathrm{h}} \tau_{\mathrm{h}}$, where $\tau_{\mathrm{h}}$ denotes a hadronically decaying $\tau$. These results are an extension of previous searches by the CMS and ATLAS experiments [15-17] at $7 \mathrm{TeV}$, and are complementary to the searches in $\mathrm{p} \overline{\mathrm{p}}$ and $\mathrm{e}^{+} \mathrm{e}^{-}$collisions at the Tevatron [18-21] and LEP [22], respectively.

The results are interpreted in the context of the MSSM with different benchmark scenarios described in section 1.1 and also in a model independent way, in terms of upper limits on the cross section times branching fraction $\sigma \cdot \mathcal{B}(\phi \rightarrow \tau \tau)$ for gluon fusion $(\operatorname{gg} \phi)$ and b-quark associated ( $\mathrm{bb} \phi)$ neutral Higgs boson production, where $\phi$ denotes a single resonance with a narrow width compared to the experimental resolution.

\subsection{MSSM Higgs boson benchmark scenarios}

Traditionally, searches for MSSM Higgs bosons are expressed in terms of benchmark scenarios where the parameters $\tan \beta$ and $m_{\mathrm{A}}$ are varied, while the other parameters that enter through radiative corrections are fixed to certain benchmark values. At tree level the masses of the neutral MSSM scalar Higgs bosons $\mathrm{h}$ and $\mathrm{H}$ can be expressed in terms of $\tan \beta$ and $m_{\mathrm{A}}$ as follows

$$
m_{\mathrm{H}, \mathrm{h}}^{2}=\frac{1}{2}\left[m_{\mathrm{A}}^{2}+m_{\mathrm{Z}}^{2} \pm \sqrt{\left(m_{\mathrm{A}}^{2}+m_{\mathrm{Z}}^{2}\right)^{2}-4 m_{\mathrm{Z}}^{2} m_{\mathrm{A}}^{2}\left(\cos ^{2} 2 \beta\right)}\right],
$$

which gives an upper bound on the light scalar Higgs boson mass, $m_{\mathrm{h}}$, in terms of the Z-boson mass of $m_{\mathrm{h}} \leq m_{\mathrm{Z}} \cos 2 \beta$, which is below the excluded value of the LEP experiments [22]. After radiative corrections, values of the mass larger than the LEP limits are obtained with a maximum value of $m_{\mathrm{h}} \sim 135 \mathrm{GeV}$ [23]. 
Taking into account higher-order corrections, the following extended set of parameters defines the MSSM Higgs sector: $M_{\text {SUSY }}$ denotes the common soft-SUSY-breaking thirdgeneration squark masses; $\mu$ is the higgsino mass parameter; $M_{1}\left(M_{2}\right)$ is the $\mathrm{U}(1)(\mathrm{SU}(2))$ gaugino mass parameter; $X_{t}$ is the stop mixing parameter; $A_{t}, A_{b}$ and $A_{\tau}$ are the trilinear Higgs-stop, Higgs-sbottom and Higgs-stau-lepton couplings, respectively; $m_{\widetilde{\mathrm{g}}}\left(m_{\tilde{l}_{3}}\right)$ is the gluino (stau) mass. $A_{t}$ is obtained by the relation $A_{t}=X_{t}+\mu / \tan \beta$ and the value of the $\mathrm{U}(1)$-gaugino mass parameter $M_{1}$ is generally fixed via the unification relation $M_{1}=$ $(5 / 3) M_{2} \tan ^{2} \theta_{w}$, where $\cos \theta_{w}=m_{\mathrm{W}} / m_{\mathrm{Z}}$.

Previous MSSM Higgs searches [15-22] were interpreted in the $m_{\mathrm{h}}^{\max }$ benchmark scenario $[24,25]$, which allows the mass of the light scalar Higgs boson $\mathrm{h}$ to reach its maximum value of $\sim 135 \mathrm{GeV}$. The ATLAS and CMS experiments have reported the observation of a new boson with mass around $125 \mathrm{GeV}$ [26-28]. Evidence that this new boson also decays into tau lepton pairs has recently been reported by CMS [29]. If the new boson is interpreted as the light scalar MSSM Higgs boson $\mathrm{h}$, a large part of the $\tan \beta$ and $m_{\mathrm{A}}$ parameter space in the $m_{\mathrm{h}}^{\max }$ scenario is excluded. However, changes in some of the parameters open up a large region of the allowed parameter space again [30]. New benchmark scenarios [31] have thus recently been proposed where the mass of one of the scalar Higgs bosons, h or $\mathrm{H}$, is compatible with the mass of the recently discovered Higgs boson of $125 \mathrm{GeV}$ within a range of $\pm 3 \mathrm{GeV}$. This uncertainty is a conservative estimate of the theoretical uncertainty of the MSSM Higgs boson mass calculations [23]. Table 1 summarizes the main parameters of the benchmark scenarios considered in this study.

The traditional $m_{\mathrm{h}}^{\max }$ scenario has been slightly modified to the $m_{\mathrm{h}}^{\bmod +}$ and $m_{\mathrm{h}}^{\bmod -}$ scenarios, where the different values of the stop mixing parameter yield a smaller light scalar Higgs boson mass than the maximal value of $\sim 135 \mathrm{GeV}$. Other scenarios which have recently been proposed due to their interesting Higgs sector phenomenology compared to the SM are the light-stop scenario, which allows for a modified gluon fusion rate; the light-stau, which gives a modified $\mathrm{H} \rightarrow \gamma \gamma$ rate; and the $\tau$-phobic scenario, which gives a reduced Higgs decay rate to down-type fermions of up to $30 \%$ at large values of $\tan \beta$ and $m_{\mathrm{A}}$. The value of $m_{\mathrm{A}}$ is generally varied between 90 and $1000 \mathrm{GeV}$. In the light-stop scenario the scan is only performed up to $600 \mathrm{GeV}$, because the calculation of the SUSY next-to-leading order (NLO) QCD corrections loses validity at larger masses. The range of $\tan \beta$ values studied for each scenario is chosen such that the calculation of the light scalar Higgs boson mass is well defined. In contrast to the other scenarios, that interpret the light scalar Higgs $\mathrm{h}$ as the recently discovered Higgs boson, the low- $m_{\mathrm{H}}$ scenario assumes the heavy scalar MSSM Higgs $\mathrm{H}$ as the new discovered state. In this scenario, the parameters have been chosen such that the mass of the light scalar Higgs $h$ is not excluded by the LEP results [22]. The mass of the pseudoscalar Higgs boson is set to $m_{\mathrm{A}}=110 \mathrm{GeV}$ and the higgsino mass parameter $\mu$ and $\tan \beta$ are varied as shown in table 1 .

The neutral MSSM Higgs boson production cross sections and the corresponding uncertainties are provided by the LHC Higgs Cross Section Group [32]. The cross sections for the gluon fusion process in the $m_{\mathrm{h}}^{\max }$ scenario have been obtained with the NLO QCD program HiGLU [33, 34], for the contribution of the top loop, the bottom loop, and the interference. The top loop contribution has been further corrected using the next-to-next- 


\begin{tabular}{|l|ccc|}
\hline Parameter & $m_{\mathrm{h}}^{\max }$ & $m_{\mathrm{h}}^{\bmod +}$ & $m_{\mathrm{h}}^{\text {mod}}-$ \\
\hline$m_{\mathrm{A}}$ & $90-1000 \mathrm{GeV}$ & $90-1000 \mathrm{GeV}$ & $90-1000 \mathrm{GeV}$ \\
$\tan \beta$ & $0.5-60$ & $0.5-60$ & $0.5-60$ \\
$M_{\mathrm{SUSY}}$ & $1000 \mathrm{GeV}$ & $1000 \mathrm{GeV}$ & $1000 \mathrm{GeV}$ \\
$\mu$ & $200 \mathrm{GeV}$ & $200 \mathrm{GeV}$ & $200 \mathrm{GeV}$ \\
$M_{1}$ & $(5 / 3) M_{2} \tan ^{2} \theta_{W}$ & $(5 / 3) M_{2} \tan ^{2} \theta_{W}$ & $(5 / 3) M_{2} \tan ^{2} \theta_{W}$ \\
$M_{2}$ & $200 \mathrm{GeV}$ & $200 \mathrm{GeV}$ & $200 \mathrm{GeV}$ \\
$X_{t}$ & $2 M_{\mathrm{SUSY}}$ & $1.5 M_{\mathrm{SUSY}}$ & $-1.9 M_{\mathrm{SUSY}}$ \\
$A_{b}, A_{t}, A_{\tau}$ & $A_{b}=A_{t}=A_{\tau}$ & $A_{b}=A_{t}=A_{\tau}$ & $A_{b}=A_{t}=A_{\tau}$ \\
$m_{\widetilde{\mathrm{g}}}$ & $1500 \mathrm{GeV}$ & $1500 \mathrm{GeV}$ & $1500 \mathrm{GeV}$ \\
$m_{\tilde{\mathrm{I}_{3}}}$ & $1000 \mathrm{GeV}$ & $1000 \mathrm{GeV}$ & $1000 \mathrm{GeV}$ \\
\hline
\end{tabular}

\begin{tabular}{|l|cccc|}
\hline Parameter & light-stop & light-stau & $\tau$-phobic & low- $m_{\mathrm{H}}$ \\
\hline$m_{\mathrm{A}}$ & $90-600 \mathrm{GeV}$ & $90-1000 \mathrm{GeV}$ & $90-1000 \mathrm{GeV}$ & $110 \mathrm{GeV}$ \\
$\tan \beta$ & $0.7-60$ & $0.5-60$ & $0.9-50$ & $1.5-9.5$ \\
$M_{\mathrm{SUSY}}$ & $500 \mathrm{GeV}$ & $1000 \mathrm{GeV}$ & $1500 \mathrm{GeV}$ & $1500 \mathrm{GeV}$ \\
$\mu$ & $400 \mathrm{GeV}$ & $500 \mathrm{GeV}$ & $2000 \mathrm{GeV}$ & $300-3100 \mathrm{GeV}$ \\
$M_{1}$ & $340 \mathrm{GeV}$ & $(5 / 3) M_{2} \tan ^{2} \theta_{W}$ & $(5 / 3) M_{2} \tan ^{2} \theta_{W}$ & $(5 / 3) M_{2} \tan ^{2} \theta_{W}$ \\
$M_{2}$ & $400 \mathrm{GeV}$ & $200 \mathrm{GeV}$ & $200 \mathrm{GeV}$ & $200 \mathrm{GeV}$ \\
$X_{t}$ & $2 M_{\mathrm{SUSY}}$ & $1.6 M_{\mathrm{SUSY}}$ & $2.45 M_{\mathrm{SUSY}}$ & $2.45 M_{\mathrm{SUSY}}$ \\
$A_{b}, A_{t}, A_{\tau}$ & $A_{b}=A_{t}=A_{\tau}$ & $A_{b}=A_{t}, A_{\tau}=0$ & $A_{b}=A_{t}=A_{\tau}$ & $A_{b}=A_{t}=A_{\tau}$ \\
$m_{\widetilde{\mathrm{g}}}$ & $1500 \mathrm{GeV}$ & $1500 \mathrm{GeV}$ & $1500 \mathrm{GeV}$ & $1500 \mathrm{GeV}$ \\
$m_{\tilde{l_{3}}}$ & $1000 \mathrm{GeV}$ & $245 \mathrm{GeV}$ & $500 \mathrm{GeV}$ & $1000 \mathrm{GeV}$ \\
\hline
\end{tabular}

Table 1. MSSM benchmark scenarios.

to-leading order (NNLO) program GGH@NNLO [35-39]. In the case of the other benchmark scenarios, the program SUSHI [40] has been used as it includes the SUSY NLO QCD corrections [41-45] that are of importance in these alternative scenarios. In the SUSHI calculations, the electroweak corrections due to light-fermion loop effects [46, 47] have also been included. For the $\mathrm{bb} \phi$ process, the four-flavor NLO QCD calculation $[48,49]$ and the five-flavor NNLO QCD calculation, as implemented in BBH@NNLO [50] have been combined using the Santander matching scheme [51]. In all cross section programs used, the Higgs boson Yukawa couplings have been calculated with FeynHiggs [23, 52-54]. The Higgs boson branching fraction to tau leptons in the different benchmark scenarios has been obtained with FEYNHigGs and HDECAY [55-57], as described in ref. [58]. 


\section{Experimental setup, event reconstruction, and simulation}

The central feature of the CMS apparatus is a superconducting solenoid of $6 \mathrm{~m}$ internal diameter, providing a magnetic field of $3.8 \mathrm{~T}$. Within the superconducting solenoid volume are a silicon pixel and strip tracker, a lead tungstate crystal electromagnetic calorimeter, and a brass/scintillator hadron calorimeter, each composed of a barrel and two endcap sections. Muons are measured in gas-ionization detectors embedded in the steel fluxreturn yoke outside the solenoid. Extensive forward calorimetry complements the coverage provided by the barrel and endcap detectors. The first level of the CMS trigger system, composed of custom hardware processors, uses information from the calorimeters and muon detectors to select the most interesting events in a fixed time interval of less than $4 \mu \mathrm{s}$. The High Level Trigger processor farm further decreases the event rate from around $100 \mathrm{kHz}$ to less than $1 \mathrm{kHz}$, before data storage. A more detailed description of the CMS detector, together with a definition of the coordinate system, can be found in ref. [14].

An average of 9 (21) pp interactions occurred per LHC bunch crossing in 2011 (2012). For each reconstructed collision vertex the sum of the $p_{\mathrm{T}}^{2}$ of all tracks associated to the vertex is computed and the one with the largest value is taken as the primary collision vertex, where $p_{\mathrm{T}}$ is the transverse momentum. The additional pp collisions are referred to as pileup.

A particle-flow algorithm $[59,60]$ is used to combine information from all CMS subdetectors to identify and reconstruct individual particles in the event, namely muons, electrons, photons, charged hadrons, and neutral hadrons. The resulting particles are used to reconstruct jets, hadronically decaying tau leptons, and the missing transverse energy vector $\vec{E}_{\mathrm{T}}^{\text {miss }}$, defined as the negative of the vector sum of the transverse momenta of all reconstructed particles, and its magnitude $E_{\mathrm{T}}^{\mathrm{miss}}$.

Jets are reconstructed using the anti- $k_{\mathrm{T}}$ jet algorithm $[61,62]$ with a distance parameter of 0.5. To correct for the contribution to the jet energy due to pileup, a median transverse momentum density $(\rho)$ is determined event by event. The pileup contribution to the jet energy is estimated as the product of $\rho$ and the area of the jet and subsequently subtracted from the jet transverse momentum [63]. Jet energy corrections [64] are also applied as a function of the jet $p_{\mathrm{T}}$ and pseudorapidity $\eta=-\ln [\tan (\theta / 2)]$, where $\theta$ is the polar angle. To tag jets coming from b-quark decays the combined secondary vertex algorithm is used, that is based on the reconstruction of secondary vertices, together with track-based lifetime information [65]. Jets with $|\eta|<4.7$ and b-tagged jets with $|\eta|<2.4$ are used.

Hadronically-decaying tau leptons are reconstructed using the hadron-plus-strips algorithm [66]. The constituents of the reconstructed jets are used to identify individual $\tau$ decay modes with one charged hadron and up to two neutral pions, or three charged hadrons. The presence of extra particles within the jet, not compatible with the reconstructed decay mode of the $\tau$, is used as a criterion to discriminate $\tau_{\mathrm{h}}$ decays from jets. Additional discriminators are used to separate $\tau_{\mathrm{h}}$ decays from electrons and muons.

Tau leptons from Higgs boson decays are expected to be isolated in the detector, while leptons from heavy-flavor (c and b) decays and decays in flight are expected to be found inside jets. A measure of isolation is used to discriminate the signal from the QCD multijet background, based on the charged hadrons, photons, and neutral hadrons falling within a 
cone around the lepton momentum direction. Electron, muon, and tau lepton isolation are estimated as

$$
\begin{aligned}
I_{\mathrm{e}, \mu} & =\sum_{\text {charged }} p_{\mathrm{T}}+\max \left(0, \sum_{\text {neutral }} p_{\mathrm{T}}+\sum_{\gamma} p_{\mathrm{T}}-0.5 \sum_{\text {charged,pileup }} p_{\mathrm{T}}\right), \\
I_{\tau_{\mathrm{h}}} & =\sum_{\text {charged }} p_{\mathrm{T}}+\max \left(0, \sum_{\gamma} p_{\mathrm{T}}-0.46 \sum_{\text {charged,pileup }} p_{\mathrm{T}}\right),
\end{aligned}
$$

where $\sum_{\text {charged }} p_{\mathrm{T}}$ is the scalar sum of the transverse momenta of the charged hadrons, electrons, and muons from the primary vertex located in a cone centered around the lepton direction of size $\Delta R=\sqrt{(\Delta \eta)^{2}+(\Delta \phi)^{2}}$ of 0.4 for electrons and muons and 0.5 for tau leptons. The sums $\sum_{\text {neutral }} p_{\mathrm{T}}$ and $\sum_{\gamma} p_{\mathrm{T}}$ represent the same quantities for neutral hadrons and photons, respectively. In the case of electrons and muons the innermost region is excluded to avoid the footprint in the calorimeter of the lepton itself from entering the sum. Charged particles close to the direction of the electrons are excluded as well, to prevent tracks originating from the conversion of photons emitted by the bremsstrahlung process from spoiling the isolation. In the case of $\tau_{\mathrm{h}}$, the particles used in the reconstruction of the lepton are excluded. The contribution of pileup photons and neutral hadrons is estimated from the scalar sum of the transverse momenta of charged hadrons from pileup

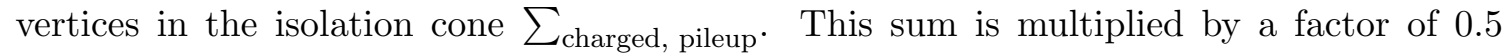
that approximately corresponds to the ratio of neutral-to-charged hadron production in the hadronization process of inelastic pp collisions. In the case of $\tau_{\mathrm{h}}$, a value of 0.46 is used, as the neutral hadron contribution is not used in the computation of $I_{\tau_{\mathrm{h}}}$. An $\eta, p_{\mathrm{T}}$, and lepton-flavor dependent threshold on the isolation variable is applied.

In order to mitigate the effects of pileup on the reconstruction of $E_{\mathrm{T}}^{\mathrm{miss}}$, a multivariate regression correction is used where the inputs are separated in those components coming from the primary vertex and those which are not [67]. The correction improves the $E_{\mathrm{T}}^{\mathrm{miss}}$ resolution in $\mathrm{Z} \rightarrow \mu \mu$ events by roughly a factor of two in the case where 25 additional pileup events are present.

The MSSM neutral Higgs boson signals are modelled with the event generator PYTHIA 6.4 [68]. For the background processes, the MADGRAPH 5.1 [69] generator is used for $\mathrm{Z}+$ jets, $\mathrm{W}+$ jets, $\mathrm{t} \overline{\mathrm{t}}$ and di-boson production, and POWHEG 1.0 [70-73] for single-top-quark production. The POWHEG and MADGRAPH generators are interfaced with PYTHIA for parton shower and fragmentation. All generators are interfaced with TAUOLA [74] for the simulation of the $\tau$ decays. Additional interactions are simulated with PYTHIA and reweighted to the observed pileup distribution in data. All generated events are processed through a detailed simulation of the CMS detector based on GEANT4 [75] and are reconstructed with the same algorithms as the data. The missing transverse energy in Monte Carlo (MC) simulated events is corrected for the difference between data and simulation measured using a sample of $\mathrm{Z} \rightarrow \mu \mu$ events [76]. 


\section{Event selection}

The events in this analysis have been selected with dedicated triggers that use a combination of electron, muon and tau lepton trigger objects [77-79]. The identification criteria and transverse momentum thresholds of these objects were progressively tightened as the LHC instantaneous luminosity increased over the data-taking period.

In the $\mathrm{e} \tau_{\mathrm{h}}$ and $\mu \tau_{\mathrm{h}}$ final states, events are selected in the 2011 (2012) dataset with an electron of $p_{\mathrm{T}}>20(24) \mathrm{GeV}$ or a muon of $p_{\mathrm{T}}>17(20) \mathrm{GeV}$ and $|\eta|<2.1$, and an oppositely charged $\tau_{\mathrm{h}}$ of $p_{\mathrm{T}}>20 \mathrm{GeV}$ and $|\eta|<2.3$. The tau lepton is required to have $I_{\tau_{\mathrm{h}}}$ of less than $1.5 \mathrm{GeV}$. To reduce the $\mathrm{Z} \rightarrow$ ee, $\mu \mu$ contamination, events with two electrons or muons of $p_{\mathrm{T}}>15 \mathrm{GeV}$, of opposite charge, and passing loose isolation criteria are rejected.

In the $\mathrm{e} \mu$ and $\mu \mu$ final states, events with two oppositely charged leptons are selected, where the highest (second-highest) $p_{\mathrm{T}}$ lepton is required to have $p_{\mathrm{T}}>20(10) \mathrm{GeV}$. Electrons with $|\eta|<2.3$ and muons with $|\eta|<2.1$ are used. The large background arising from $\mathrm{Z} \rightarrow \mu \mu$ events in the $\mu \mu$ channel is reduced by a multivariate boosted decision tree discriminator [80] using different muon kinematic variables, including the distance of closest approach of the muon pair.

In the $\tau_{\mathrm{h}} \tau_{\mathrm{h}}$ final state, events with two oppositely charged hadronically decaying tau leptons with $p_{\mathrm{T}}>45 \mathrm{GeV}$ and $|\eta|<2.1$ are selected, where the isolation $I_{\tau_{\mathrm{h}}}$ of both tau leptons is required to be less than $1 \mathrm{GeV}$.

In order to reject events coming from the $\mathrm{W}+$ jets background, a dedicated selection is applied. In the $\mathrm{e} \tau_{\mathrm{h}}$ and $\mu \tau_{\mathrm{h}}$ final states, the transverse mass of the electron or muon and the $E_{\mathrm{T}}^{\mathrm{miss}}$

$$
M_{T}=\sqrt{2 p_{\mathrm{T}} E_{\mathrm{T}}^{\mathrm{miss}}(1-\cos \Delta \phi)},
$$

is required to be less than $30 \mathrm{GeV}$, where $p_{\mathrm{T}}$ is the lepton transverse momentum and $\Delta \phi$ is the difference in the azimuthal angle between the lepton momentum and the $\vec{E}_{\mathrm{T}}^{\text {miss }}$. In the $\mathrm{e} \mu$ final state, a discriminator to reject $\mathrm{W}+$ jets events is formed by considering the bisector of the directions of the visible $\tau$ decay products transverse to the beam direction, and is denoted as the $\zeta$ axis. From the projections of the visible decay product momenta and the $\vec{E}_{\mathrm{T}}^{\text {miss }}$ onto the $\zeta$ axis, two values are calculated,

$$
P_{\zeta}=\left(\vec{p}_{\mathrm{T}, 1}+\vec{p}_{\mathrm{T}, 2}+\vec{E}_{\mathrm{T}}^{\mathrm{miss}}\right) \cdot \frac{\vec{\zeta}}{|\zeta|} \quad \text { and } \quad P_{\zeta}^{\mathrm{vis}}=\left(\vec{p}_{\mathrm{T}, 1}+\vec{p}_{\mathrm{T}, 2}\right) \cdot \frac{\vec{\zeta}}{|\zeta|},
$$

where $p_{\mathrm{T}, 1}$ and $p_{\mathrm{T}, 2}$ indicate the transverse momentum of the two reconstructed leptons. Events are selected with $P_{\zeta}-1.85 P_{\zeta}^{\text {vis }}>-20 \mathrm{GeV}$.

To further enhance the sensitivity of the search to Higgs bosons, the sample of selected events is split into two mutually exclusive categories:

- b-tag: at least one b-tagged jet with $p_{\mathrm{T}}>20 \mathrm{GeV}$ is required and not more than

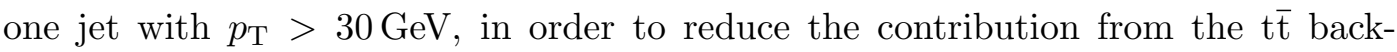
ground. This event category is intended to exploit the production of Higgs bosons in association with b quarks which is enhanced in the MSSM. 
- no b-tag: events are required to have no b-tagged jets with $p_{\mathrm{T}}>20 \mathrm{GeV}$. This event category is mainly sensitive to the gluon fusion Higgs boson production mechanism.

This analysis uses a simpler event categorization than the dedicated SM Higgs boson search in the $\tau \tau$ decay mode [29], to reduce possible model dependencies in the result interpretation. The sensitivity to the SM Higgs boson in this analysis is thus reduced, as the contributions from vector boson fusion and boosted gluon fusion Higgs boson production are not enhanced.

\section{Background estimation}

The estimation of the shapes and yields of the major backgrounds in each of the channels is obtained from the observed data.

The $\mathrm{Z} \rightarrow \tau \tau$ process is the largest source of background events in the $\mathrm{e} \tau_{\mathrm{h}}, \mu \tau_{\mathrm{h}}$, and $\mathrm{e} \mu$ channels. This background is estimated using a sample of $\mathrm{Z} \rightarrow \mu \mu$ events from data where the reconstructed muons are replaced by the reconstructed particles from simulated $\tau$ decays. The normalization for this process is determined from the measurement of the $\mathrm{Z} \rightarrow \mu \mu$ yield in data. This technique substantially reduces the systematic uncertainties due to the jet energy scale and the missing transverse energy, as these quantities are modelled with collision data.

Another significant source of background is QCD multijet events, which can mimic the signal in various ways. For example, two jets may be misidentified as $\tau_{\mathrm{h}}$ decays in which case the event will contribute to the $\tau_{\mathrm{h}} \tau_{\mathrm{h}}$ channel. Or, in the $\mathrm{e} \tau_{\mathrm{h}}$ and $\mu \tau_{\mathrm{h}}$ channels, one jet is misidentified as an isolated electron or muon and a second jet as $\tau_{\mathrm{h}}$. In the $\mathrm{e} \tau_{\mathrm{h}}$ and $\mu \tau_{\mathrm{h}}$ channels, the shape of the QCD background is estimated using a sample of same-sign (SS) $\tau \tau$ events in data. The yield is obtained by scaling the observed number of SS events by the ratio of the opposite-sign (OS) to SS event yields obtained in a QCD-enriched region with loose lepton isolation. In the $\tau_{\mathrm{h}} \tau_{\mathrm{h}}$ channel, the shape is obtained from OS events with loose $\tau$ isolation. The yield is obtained by scaling these events by the ratio of SS events with tight and loose $\tau$ isolation.

$\mathrm{W}+$ jets events in which there is a jet misidentified as a $\tau_{\mathrm{h}}$ are another sizable source of background in the $\mathrm{e} \tau_{\mathrm{h}}$ and $\mu \tau_{\mathrm{h}}$ channels. The background shape is modelled using a MC simulation and the rate is estimated using a control region of events with large transverse mass.

The Drell-Yan production of muon pairs is the largest background in the $\mu \mu$ channel. The $\mathrm{Z} \rightarrow \mu \mu$ event yield is obtained from a fit to the distance of closest approach of the muon pairs observed in data, after subtracting all backgrounds. In the $\mathrm{e} \tau_{\mathrm{h}}$ and $\mu \tau_{\mathrm{h}}$ channels, the contribution of Drell-Yan production of electron and muon pairs is estimated from the simulation, after rescaling the simulated yield to the one derived from $\mathrm{Z} \rightarrow \mu \mu$ data. In the $\mathrm{e} \tau_{\mathrm{h}}$ channel, the $\mathrm{Z} \rightarrow$ ee simulation is further corrected using the $\mathrm{e} \rightarrow \tau_{\mathrm{h}}$ fake-rate measured in data using a "tag-and-probe" technique [76] on $\mathrm{Z} \rightarrow$ ee events.

In the $\mathrm{e} \mu$ final state, the $\mathrm{W}+$ jets and multijet background rate is obtained by measuring the number of events with one good lepton and a second one that passes relaxed selection 


\begin{tabular}{|lcccc|}
\hline \multicolumn{5}{c|}{ e $\tau_{\mathrm{h}}$ channel } \\
\hline \multicolumn{2}{c}{$\sqrt{s}=7 \mathrm{TeV}$} & \multicolumn{2}{c|}{$\sqrt{s}=8 \mathrm{TeV}$} \\
Process & no b-tag & b-tag & no b-tag & b-tag \\
\hline $\mathrm{Z} \rightarrow \tau \tau$ & $11819 \pm 197$ & $135 \pm 4$ & $30190 \pm 345$ & $453 \pm 14$ \\
$\mathrm{QCD}$ & $4163 \pm 212$ & $78 \pm 11$ & $11894 \pm 544$ & $194 \pm 27$ \\
$\mathrm{~W}+$ jets & $1344 \pm 112$ & $29 \pm 7$ & $5646 \pm 385$ & $113 \pm 25$ \\
$\mathrm{Z}+$ jets $(\mathrm{e}, \mu$ or jet faking $\tau)$ & $1334 \pm 130$ & $9 \pm 1$ & $6221 \pm 360$ & $83 \pm 7$ \\
$\mathrm{t} \overline{\mathrm{t}}$ & $43 \pm 3$ & $19 \pm 3$ & $290 \pm 22$ & $102 \pm 12$ \\
Di-bosons + single top & $46 \pm 5$ & $7 \pm 0.9$ & $224 \pm 23$ & $30 \pm 4$ \\
\hline Total background & $18750 \pm 144$ & $278 \pm 11$ & $54464 \pm 259$ & $975 \pm 29$ \\
$\mathrm{~h}, \mathrm{H}, \mathrm{A} \rightarrow \tau \tau$ & $128 \pm 13$ & $10 \pm 1$ & $466 \pm 43$ & $37 \pm 5$ \\
Observed data & 18785 & 274 & 54547 & 975 \\
\hline Efficiency $\times$ acceptance & & \multicolumn{3}{c}{} \\
\hline gluon fusion Higgs & $1.39 \times 10^{-2}$ & $1.24 \times 10^{-4}$ & $9.48 \times 10^{-3}$ & $1.11 \times 10^{-4}$ \\
b-quark associated Higgs & $1.12 \times 10^{-2}$ & $2.10 \times 10^{-3}$ & $7.78 \times 10^{-3}$ & $1.49 \times 10^{-3}$ \\
\hline
\end{tabular}

Table 2. Observed and expected number of events in the two event categories in the e $\tau_{\mathrm{h}}$ channel, where the combined statistical and systematic uncertainty is shown. The expected signal yields for $\mathrm{h}, \mathrm{H}, \mathrm{A} \rightarrow \tau \tau$ in the $m_{\mathrm{h}}^{\max }$ scenario for $m_{\mathrm{A}}=160 \mathrm{GeV}$ and $\tan \beta=8$ and the signal efficiency times acceptance for a MSSM Higgs boson of $160 \mathrm{GeV}$ mass are also given.

criteria, but fails the nominal lepton selection. This rate is extrapolated to the signal region using the efficiencies for such loose lepton candidates to pass the nominal lepton selection. These efficiencies are measured in data using multijet events.

The $t \bar{t}$, di-boson and single-top-quark background contributions are estimated from

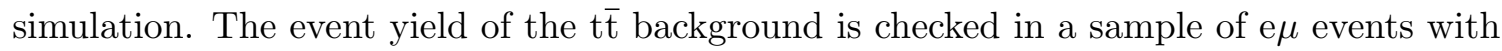
two b-tagged jets.

The observed number of events for each category, the expected number of events from various background processes, and the expected signal yields and efficiencies, are shown in tables 2-4. The uncertainties are obtained after the likelihood fit described in section 7 .

\section{$5 \quad$ Tau lepton-pair invariant mass}

To distinguish Higgs boson signals from the background, the tau-lepton pair invariant mass, $m_{\tau \tau}$, is reconstructed using a maximum likelihood technique [29]. The $m_{\tau \tau}$ resolution for $\mathrm{Z} \rightarrow \tau \tau$ events depends on the final state considered but typically amounts to $20 \%$, relative to the true mass value. Distributions of the mass of the visible decay products $m_{\text {vis }}$ and $m_{\tau \tau}$ for simulated events are shown in figure 2. The reconstruction of $m_{\tau \tau}$ improves the separation power between the main $\mathrm{Z} \rightarrow \tau \tau$ background and the hypothetical MSSM Higgs boson A signals.

The distribution in $m_{\tau \tau}$ for the five final states studied, $\mathrm{e} \tau_{\mathrm{h}}, \mu \tau_{\mathrm{h}}, \mathrm{e} \mu, \mu \mu$, and $\tau_{\mathrm{h}} \tau_{\mathrm{h}}$, compared with the background prediction in the no b-tag category is shown in figure 3. 


\begin{tabular}{|c|c|c|c|c|}
\hline \multicolumn{5}{|c|}{$\mu \tau_{\mathrm{h}}$ channel } \\
\hline \multirow[b]{2}{*}{ Process } & \multicolumn{2}{|c|}{$\sqrt{s}=7 \mathrm{TeV}$} & \multicolumn{2}{|c|}{$\sqrt{s}=8 \mathrm{TeV}$} \\
\hline & no b-tag & b-tag & no b-tag & b-tag \\
\hline $\mathrm{Z} \rightarrow \tau \tau$ & $26838 \pm 244$ & $284 \pm 8$ & $87399 \pm 497$ & $1118 \pm 31$ \\
\hline QCD & $5495 \pm 258$ & $131 \pm 18$ & $18056 \pm 811$ & $552 \pm 62$ \\
\hline $\mathrm{W}+$ jets & $2779 \pm 201$ & $55 \pm 14$ & $12845 \pm 793$ & $237 \pm 52$ \\
\hline $\mathrm{Z}+$ jets $(\mathrm{e}, \mu$ or jet faking $\tau$ ) & $716 \pm 109$ & $11 \pm 2$ & $3704 \pm 454$ & $54 \pm 9$ \\
\hline $\mathrm{t} \overline{\mathrm{t}}$ & $82 \pm 6$ & $36 \pm 5$ & $564 \pm 41$ & $194 \pm 22$ \\
\hline Di-bosons + single top & $94 \pm 11$ & $12 \pm 2$ & $506 \pm 51$ & $60 \pm 7$ \\
\hline Total background & $36004 \pm 205$ & $530 \pm 18$ & $123075 \pm 407$ & $2214 \pm 44$ \\
\hline $\mathrm{h}, \mathrm{H}, \mathrm{A} \rightarrow \tau \tau$ & $226 \pm 23$ & $17 \pm 2$ & $929 \pm 85$ & $67 \pm 9$ \\
\hline Observed data & 36055 & 542 & 123239 & 2219 \\
\hline \multicolumn{5}{|l|}{ Efficiency $\times$ acceptance } \\
\hline gluon fusion Higgs & $2.34 \times 10^{-2}$ & $2.49 \times 10^{-4}$ & $1.78 \times 10^{-2}$ & $2.32 \times 10^{-4}$ \\
\hline b-quark associated Higgs & $1.96 \times 10^{-2}$ & $3.54 \times 10^{-3}$ & $1.53 \times 10^{-2}$ & $2.66 \times 10^{-3}$ \\
\hline
\end{tabular}

\begin{tabular}{|c|c|c|c|c|}
\hline \multicolumn{5}{|c|}{$\mathrm{e} \mu$ channel } \\
\hline \multirow[b]{2}{*}{ Process } & \multicolumn{2}{|c|}{$\sqrt{s}=7 \mathrm{TeV}$} & \multicolumn{2}{|c|}{$\sqrt{s}=8 \mathrm{TeV}$} \\
\hline & no b-tag & b-tag & no b-tag & b-tag \\
\hline $\mathrm{Z} \rightarrow \tau \tau$ & $13783 \pm 134$ & $165 \pm 5$ & $48218 \pm 300$ & $679 \pm 8$ \\
\hline QCD & $804 \pm 114$ & $14 \pm 3$ & $4302 \pm 356$ & $148 \pm 17$ \\
\hline $\mathrm{t} \overline{\mathrm{t}}$ & $467 \pm 29$ & $309 \pm 18$ & $2215 \pm 158$ & $1183 \pm 48$ \\
\hline Di-bosons + single top & $501 \pm 55$ & $63 \pm 8$ & $2367 \pm 248$ & $308 \pm 38$ \\
\hline Total background & $15556 \pm 128$ & $551 \pm 21$ & $57102 \pm 257$ & $2318 \pm 37$ \\
\hline $\mathrm{h}, \mathrm{H}, \mathrm{A} \rightarrow \tau \tau$ & $114 \pm 11$ & $9 \pm 1$ & $455 \pm 43$ & $34 \pm 4$ \\
\hline Observed data & 15436 & 558 & 57285 & 2353 \\
\hline \multicolumn{5}{|l|}{ Efficiency $\times$ acceptance } \\
\hline gluon fusion Higgs & $1.14 \times 10^{-2}$ & $1.11 \times 10^{-4}$ & $8.83 \times 10^{-3}$ & $8.85 \times 10^{-5}$ \\
\hline b-quark associated Higgs & $9.70 \times 10^{-3}$ & $1.80 \times 10^{-3}$ & $7.59 \times 10^{-3}$ & $1.33 \times 10^{-3}$ \\
\hline
\end{tabular}

\begin{tabular}{|c|c|c|c|c|}
\hline \multicolumn{5}{|c|}{$\mu \mu$ channel } \\
\hline & \multicolumn{2}{|c|}{$\sqrt{s}=7 \mathrm{TeV}$} & \multicolumn{2}{|c|}{$\sqrt{s}=8 \mathrm{TeV}$} \\
\hline Process & no b-tag & b-tag & no b-tag & b-tag \\
\hline $\mathrm{Z} \rightarrow \tau \tau$ & $6838 \pm 118$ & $34 \pm 1$ & $20931 \pm 376$ & $101 \pm 5$ \\
\hline $\mathrm{Z} \rightarrow \mu \mu$ & $562008 \pm 764$ & $1435 \pm 34$ & $1894509 \pm 1566$ & $5125 \pm 69$ \\
\hline $\mathrm{QCD}$ & $380 \pm 51$ & $4 \pm 2$ & $1131 \pm 108$ & $31 \pm 7$ \\
\hline $\mathrm{t} \overline{\mathrm{t}}$ & $183 \pm 15$ & $83 \pm 7$ & $809 \pm 62$ & $324 \pm 15$ \\
\hline Di-bosons + single top & $1114 \pm 221$ & $10 \pm 2$ & $5543 \pm 625$ & $48 \pm 8$ \\
\hline Total background & $570523 \pm 763$ & $1566 \pm 35$ & $1922923 \pm 1439$ & $5629 \pm 72$ \\
\hline $\mathrm{h}, \mathrm{H}, \mathrm{A} \rightarrow \tau \tau$ & $57 \pm 5$ & $3 \pm 0.4$ & $195 \pm 17$ & $8 \pm 1$ \\
\hline Observed data & 570616 & 1559 & 1922924 & 5608 \\
\hline \multicolumn{5}{|l|}{ Efficiency $\times$ acceptance } \\
\hline gluon fusion Higgs & $5.66 \times 10^{-3}$ & $2.55 \times 10^{-5}$ & $3.73 \times 10^{-3}$ & $2.29 \times 10^{-5}$ \\
\hline b-quark associated Higgs & $5.33 \times 10^{-3}$ & $6.65 \times 10^{-4}$ & $3.58 \times 10^{-3}$ & $3.42 \times 10^{-4}$ \\
\hline
\end{tabular}

Table 3. Observed and expected number of events in the two event categories in the $\mu \tau_{\mathrm{h}}, \mathrm{e} \mu$, and $\mu \mu$ channels. Other details are as in table 2. 


\begin{tabular}{|lcc|}
\hline \multicolumn{3}{|c|}{$\tau_{\mathrm{h}} \tau_{\mathrm{h}}$ channel } \\
\hline \multicolumn{3}{|c|}{$\sqrt{s}=8 \mathrm{TeV}$} \\
Process & no b-tag & b-tag \\
\hline $\mathrm{Z} \rightarrow \tau \tau$ & $2511 \pm 97$ & $60 \pm 3$ \\
$\mathrm{QCD}$ & $20192 \pm 236$ & $273 \pm 21$ \\
$\mathrm{~W}+$ jets & $630 \pm 165$ & $17 \pm 5$ \\
$\mathrm{Z}+$ jets $(\mathrm{e}, \mu$ or jet faking $\tau)$ & $115 \pm 19$ & $2 \pm 0.4$ \\
$\mathrm{t} \overline{\mathrm{t}}$ & $38 \pm 4$ & $16 \pm 2$ \\
Di-bosons + single top & $63 \pm 13$ & $5 \pm 1$ \\
\hline Total background & $23548 \pm 174$ & $374 \pm 19$ \\
$\mathrm{~h}, \mathrm{H}, \mathrm{A} \rightarrow \tau \tau$ & $325 \pm 34$ & $30 \pm 5$ \\
Observed data & 23606 & 381 \\
\hline Efficiency $\times$ acceptance & & \\
\hline gluon fusion Higgs & $9.11 \times 10^{-3}$ & $1.32 \times 10^{-4}$ \\
b-quark associated Higgs & $7.26 \times 10^{-3}$ & $1.37 \times 10^{-3}$ \\
\hline
\end{tabular}

Table 4. Observed and expected number of events in the two event categories in the $\tau_{\mathrm{h}} \tau_{\mathrm{h}}$ channel. Other details are as in table 2 .
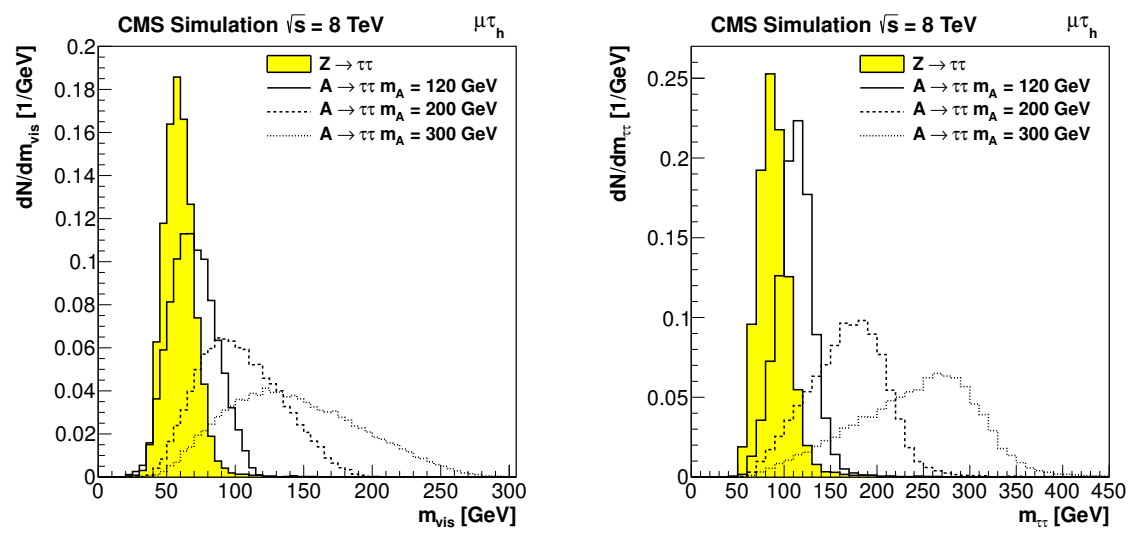

Figure 2. Visible mass $m_{\text {vis }}$ (left) and $m_{\tau \tau}$ reconstructed mass (right) for simulated events: $\mathrm{Z} \rightarrow \tau \tau$ and MSSM $\mathrm{A} \rightarrow \tau \tau$ signal with $m_{\mathrm{A}}=120,200$ and $300 \mathrm{GeV}$, in the $\mu \tau_{\mathrm{h}}$ final state. Distributions are normalized to unity.

These events are more sensitive to the gluon fusion Higgs boson production mechanism. Figure 4 shows the $m_{\tau \tau}$ distribution in the b-tag category, which has an enhanced sensitivity to the b-quark associated Higgs boson production mechanism.

\section{Systematic uncertainties}

Various imperfectly known effects can alter the shape and the normalization of the invariant-mass spectrum. The main contributions to the normalization uncertainty, that affect the signal and the simulated backgrounds, include the uncertainty in the total in- 

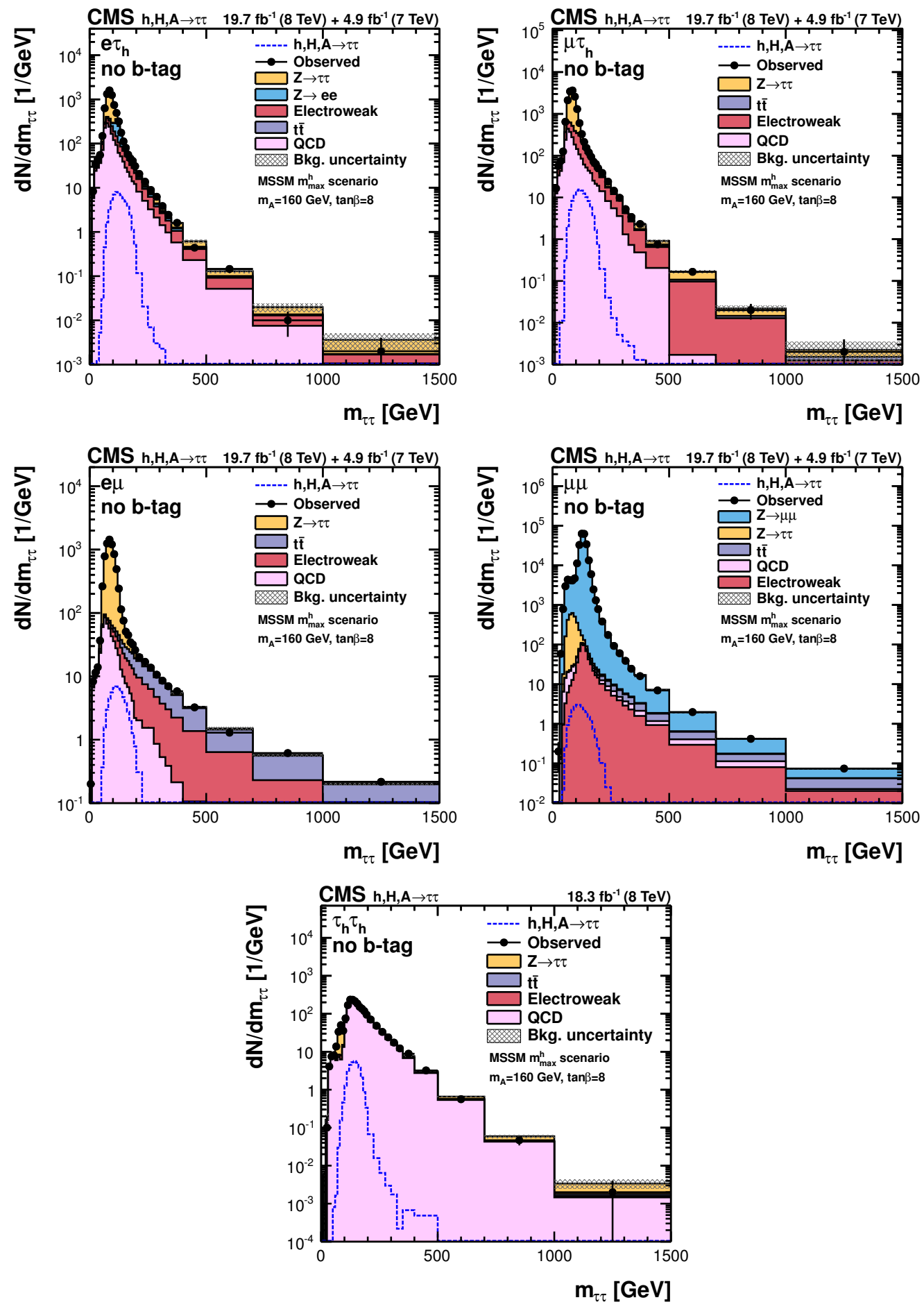

Figure 3. Reconstructed $\tau \tau$ invariant-mass in the no b-tag category for the $\mathrm{e} \tau_{\mathrm{h}}, \mu \tau_{\mathrm{h}}, \mathrm{e} \mu, \mu \mu$ and $\tau_{\mathrm{h}} \tau_{\mathrm{h}}$ channels. The electroweak background includes the contributions from $\mathrm{Z} \rightarrow$ ee, $\mathrm{Z} \rightarrow \mu \mu, \mathrm{W}$, di-bosons, and single top. In the $\mu \mu$ channel, the $\mathrm{Z} \rightarrow \mu \mu$ contribution is shown separately. The expected signal yield of the MSSM Higgs bosons $\mathrm{h}, \mathrm{H}$, and A for $m_{\mathrm{A}}=160 \mathrm{GeV}$ and $\tan \beta=8$ in the $m_{\mathrm{h}}^{\max }$ scenario is also shown. 

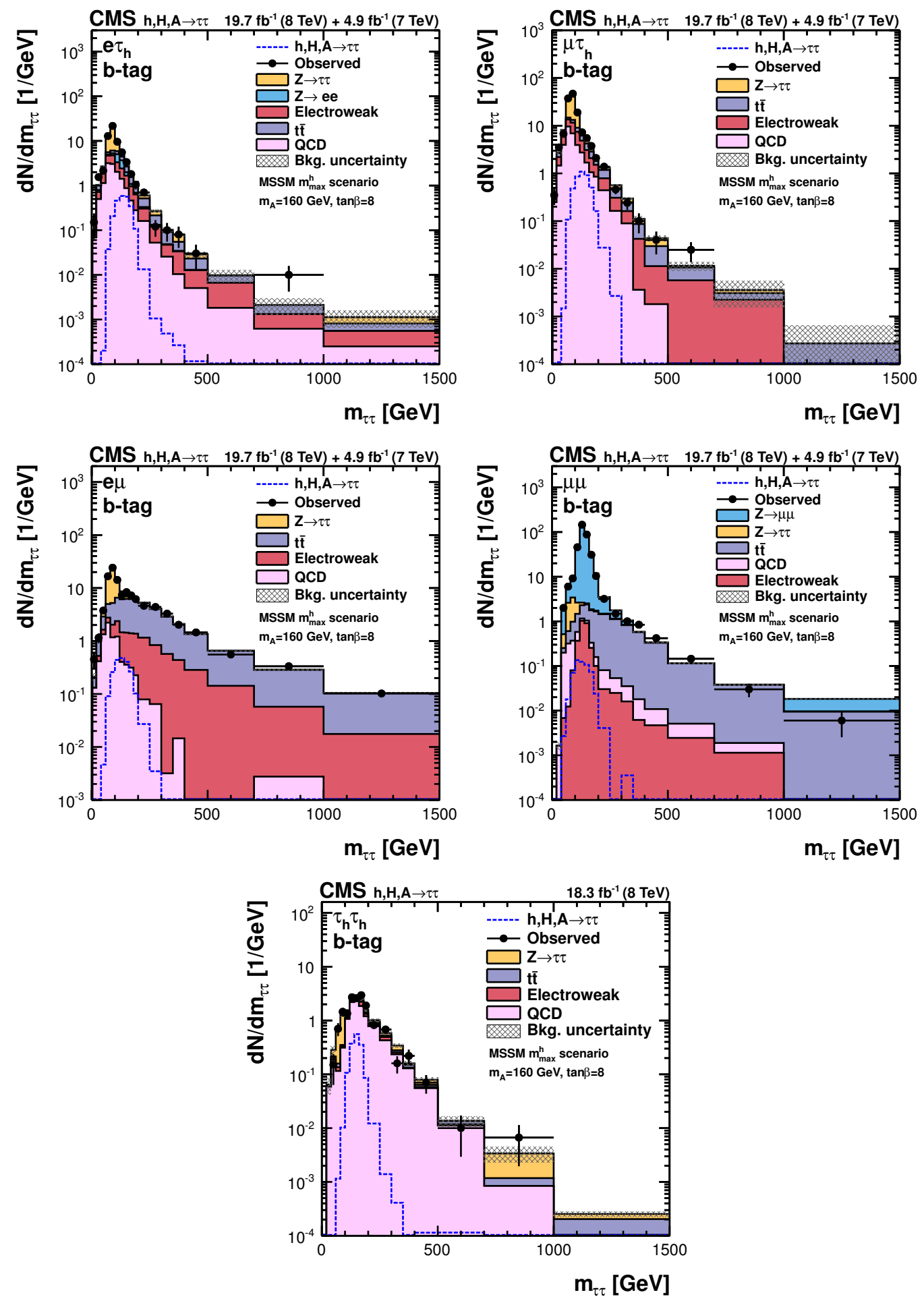

Figure 4. Reconstructed $\tau \tau$ mass in the b-tag category for the $\mathrm{e} \tau_{\mathrm{h}}, \mu \tau_{\mathrm{h}}, \mathrm{e} \mu, \mu \mu$ and $\tau_{\mathrm{h}} \tau_{\mathrm{h}}$ channels. Other details are as in figure 3. 
tegrated luminosity ( $2.2 \%$ for 2011 and $2.6 \%$ for 2012 data [81, 82]), the jet energy scale (1-10\%), and the identification and trigger efficiencies of electrons (2\%) and muons (2-3\%). The tau-lepton identification and trigger efficiency uncertainty is estimated to be $8 \%$ from an independent study done using a "tag-and-probe" technique on $\mathrm{Z} \rightarrow \tau \tau$ events. An extra uncertainty of $0.02 \% \times p_{\mathrm{T}}^{\tau}[\mathrm{GeV}]$, due to the extrapolation from the Z-boson resonance region to larger tau lepton $p_{\mathrm{T}}$ values, is also considered. The b-tagging efficiency has an uncertainty between $2-7 \%$, and the mistag rate for light-flavor partons is accurate to $10-20 \%$ [65]. The background normalization uncertainties from the estimation methods discussed in section 4 are also considered. Uncertainties that contribute to variations in the shape of the mass spectrum include the electron (1\%), muon (1\%), and tau lepton $(3 \%)$ energy scales. The main experimental uncertainties and their effect on the yields in the two event categories are summarized in table 5.

The theoretical uncertainties on the MSSM Higgs signal cross sections depend on $\tan \beta$, $m_{\mathrm{A}}$, and the scenario considered, and can amount up to $\sim 25 \%$. In the cross section calculations the MSTW2008 [83] parton distribution functions are used, and the recommended prescription $[83,84]$ to compute the uncertainties is followed. The renormalization and factorization scales used in the theoretical calculations and the variation considered are summarized in ref. [32].

\section{Statistical analysis}

To search for the presence of a MSSM Higgs boson signal in the selected events, a binned maximum likelihood fit is performed. The invariant mass of the tau-lepton pairs is used as the input to the fit in the $\mathrm{e} \tau_{\mathrm{h}}, \mu \tau_{\mathrm{h}}, \mathrm{e} \mu$, and $\tau_{\mathrm{h}} \tau_{\mathrm{h}}$ final states. The sensitivity of the $\mu \mu$ channel is enhanced by fitting the two-dimensional distribution of $m_{\tau \tau}$ versus the mass of the visible decay products, $m_{\mathrm{vis}}$, utilizing the fact that most of the large $\mathrm{Z} \rightarrow \mu \mu$ background contributing to this channel is concentrated in the $m_{\mathrm{vis}}$ distribution within a narrow peak around the Z-boson mass. The fit is performed simultaneously for the five final states and the two event categories, b-tag and no b-tag.

The systematic uncertainties described in section 6 are incorporated in the fit via nuisance parameters and are treated according to the frequentist paradigm, as described in ref. [85]. The uncertainties that affect the shape of the mass spectrum, mainly those corresponding to the energy scales, are represented by the nuisance parameters whose variation results in a continuous perturbation of the spectrum. Shape uncertainties due to limited statistics are incorporated via nuisance parameters that allow for uncorrelated single-bin fluctuations of the background expectation, following the method described in ref. [86]. In the tail of the $m_{\tau \tau}$ distribution $\left(m_{\tau \tau}>150 \mathrm{GeV}\right)$, where the statistical uncertainties are large, a different method is used. A fit of the form $f=\exp \left[-m_{\tau \tau} /\left(c_{0}+c_{1} \cdot m_{\tau \tau}\right)\right]$ is performed for each of the major backgrounds, the result of which replaces the nominal distribution. The uncertainties in the fit parameters $c_{0}$ and $c_{1}$ are treated as nuisance parameters in the likelihood fit.

The invariant-mass spectra show no clear evidence for the presence of a MSSM Higgs boson signal so exclusion limits are obtained. For the calculation of exclusion limits a 


\begin{tabular}{|c|c|c|c|}
\hline \multirow{2}{*}{ Experimental uncertainties } & \multirow[b]{2}{*}{ Uncertainty } & \multicolumn{2}{|c|}{$\begin{array}{l}\text { Event yield uncertainty } \\
\text { by event category }\end{array}$} \\
\hline & & no b-tag & b-tag \\
\hline Integrated luminosity 7 (8) TeV & $2.2(2.6) \%$ & $2.2(2.6) \%$ & $2.2(2.6) \%$ \\
\hline Jet energy scale & $1-10 \%$ & $1-5 \%$ & $1-8 \%$ \\
\hline$E_{\mathrm{T}}^{\mathrm{miss}}$ & $1-5 \%$ & $1-2 \%$ & $1-2 \%$ \\
\hline Electron identification and trigger & $2 \%$ & $2 \%$ & $2 \%$ \\
\hline Muon identification and trigger & $2-3 \%$ & $2-6 \%$ & $2-6 \%$ \\
\hline Tau-lepton identification and trigger & $8 \%$ & $8-19 \%$ & $8-19 \%$ \\
\hline b-tagging efficiency & $2-7 \%$ & $2-4 \%$ & $2-9 \%$ \\
\hline b-mistag rate & $10-20 \%$ & $2 \%$ & $2-5 \%$ \\
\hline Normalization, Z production & $3.3 \%$ & $3.3 \%$ & $3.3 \%$ \\
\hline$Z \rightarrow \tau \tau:$ category selection & $3 \%$ & - & $1-3 \%$ \\
\hline Normalization, $t \bar{t}$ & $10 \%$ & $10 \%$ & $10-17 \%$ \\
\hline Normalization, di-boson & $15-30 \%$ & $15-30 \%$ & $15-30 \%$ \\
\hline Normalization, QCD Multijet & $10-35 \%$ & $10-35 \%$ & $20-35 \%$ \\
\hline Normalization, $\mathrm{W}+$ jets & $10-30 \%$ & $10-30 \%$ & $30 \%$ \\
\hline Normalization, $\mathrm{Z} \rightarrow$ ee: e misidentified as $\tau_{\mathrm{h}}$ & $20 \%$ & $20 \%$ & $20 \%$ \\
\hline Normalization, $\mathrm{Z} \rightarrow \mu \mu: \mu$ misidentified as $\tau_{\mathrm{h}}$ & $30 \%$ & $30 \%$ & $30 \%$ \\
\hline Normalization, $\mathrm{Z}+$ jets: jet misidentified as $\tau_{\mathrm{h}}$ & $20 \%$ & $20 \%$ & $20 \%$ \\
\hline Electron energy scale & $1 \%$ & $1 \%$ & $1 \%$ \\
\hline Muon energy scale & $1 \%$ & $1 \%$ & $1 \%$ \\
\hline Tau-lepton energy scale & $3 \%$ & $3 \%$ & $3 \%$ \\
\hline
\end{tabular}

Table 5. Systematic uncertainties that affect the estimated number of signal or background events. Several uncertainties are treated as correlated for the different decay channels and event categories. Some uncertainties vary with $p_{\mathrm{T}}, \eta$, and final state, so ranges are given.

modified frequentist criterion $\mathrm{CL}_{\mathrm{s}}[87,88]$ is used. The chosen test statistic $q$, used to determine how signal- or background-like the data are, is based on a profile likelihood ratio. In this study, two searches are performed:

- a model independent search for a single narrow resonance $\phi$ for different mass hypotheses in the gluon fusion and b-quark associated Higgs boson production modes;

- a search for the MSSM neutral Higgs bosons, h, A, and H in the $\tau \tau$ mass spectrum.

In the case of the model independent search for a single resonance $\phi$, the profile likelihood ratio is defined as

$$
q_{\mu}=-2 \ln \frac{L\left(N_{\mathrm{obs}} \mid \mu \cdot s+b, \hat{\theta}_{\mu}\right)}{L\left(N_{\mathrm{obs}} \mid \hat{\mu} \cdot s+b, \hat{\theta}\right)}, \quad \text { with } 0 \leq \hat{\mu} \leq \mu,
$$

where $N_{\text {obs }}$ is the number of observed events, $b$ and $s$ are the number of expected background and signal events, $\mu$ is the signal strength modifier, and $\theta$ are the nuisance parameters describing the systematic uncertainties. The value $\hat{\theta}_{\mu}$ maximizes the likelihood in the 
numerator for a given $\mu$, while $\hat{\mu}$ and $\hat{\theta}$ define the point at which the likelihood reaches its global maximum. The ratio of probabilities to observe a value of the test statistic at least as large as the one observed in data, $q_{\mu}^{\text {obs }}$, under the signal-plus-background $(\mu \cdot s+b)$ and background-only hypotheses,

$$
\mathrm{CL}_{\mathrm{s}}(\mu)=\frac{P\left(q_{\mu} \geq q_{\mu}^{\mathrm{obs}} \mid \mu \cdot s+b\right)}{P\left(q_{\mu} \geq q_{\mu}^{\mathrm{obs}} \mid b\right)} \leq \alpha,
$$

is used as the criterion for excluding the presence of a signal at the $1-\alpha$ confidence level (CL).

Upper limits on $\sigma \cdot \mathcal{B}(\phi \rightarrow \tau \tau)$ at $95 \%$ CL for gluon fusion and b-quark associated neutral Higgs boson production for a single narrow resonance are obtained using eqs. (7.1) and (7.2). The expected limit is obtained by replacing the observed data by a representative dataset which not only contains the contribution from background processes but also a SM Higgs boson with a mass of $125 \mathrm{GeV}$. To extract the limit on the gluon fusion (b-quark associated) Higgs boson production, the rate of the b-quark associated (gluon fusion) Higgs boson production is treated as a nuisance parameter in the fit.

A search for MSSM Higgs bosons in the $\tau \tau$ final state is also performed, where the three neutral MSSM Higgs bosons are present in the signal. In light of the recent Higgs boson discovery at $125 \mathrm{GeV}$, a search for a MSSM signal versus a background-only hypothesis has lost validity. A modified $\mathrm{CL}_{\mathrm{s}}$ approach has been also adopted in this case, which tests the compatibility of the data to a signal of the three neutral Higgs bosons $\mathrm{h}, \mathrm{H}$, and A compared to a SM Higgs boson hypothesis, with inclusion of the backgrounds in both cases. To achieve this a physics model is built according to

$$
M(\mu)=[\mu \cdot s(\mathrm{MSSM})+(1-\mu) \cdot s(\mathrm{SM})]+b .
$$

In the search, two well defined theories are tested so $\mu$ can only take the value of 0 or 1 . The test statistic used in the $\mathrm{CL}_{\mathrm{s}}$ method is given by the ratio of likelihoods

$$
q_{\mathrm{MSSM} / \mathrm{SM}}=-2 \ln \frac{L\left(N_{\mathrm{obs}} \mid M(1), \hat{\theta}_{1}\right)}{L\left(N_{\mathrm{obs}} \mid M(0), \hat{\theta}_{0}\right)},
$$

where the numerator and denominator are maximized by finding the corresponding nuisance parameters $\hat{\theta}_{1}$ for $\mu=1$ and $\hat{\theta}_{0}$ for $\mu=0$.

The MSSM Higgs boson signal expectation for each benchmark scenario studied is determined in each point of the parameter space as follows:

- At each point of $m_{\mathrm{A}}$ and $\tan \beta$ : the mass, the gluon fusion and associated-b production cross sections, and the branching fraction to $\tau \tau$ are determined for $\mathrm{h}, \mathrm{H}$ and $\mathrm{A}$.

- The contributions of all three neutral Higgs boson are added using the corresponding cross sections times branching fractions.

Limits on $\tan \beta$ versus $m_{\mathrm{A}}$ at $95 \%$ CL are obtained for different benchmark MSSM scenarios following the test statistic given in eq. (7.4). 


\section{Results}

The invariant-mass spectra show no clear evidence for the presence of a MSSM Higgs boson signal. Therefore 95\% CL upper bounds on $\tan \beta$ as a function of the pseudoscalar Higgs boson mass $m_{\mathrm{A}}$ are set for the traditional MSSM benchmark scenario $m_{\mathrm{h}}^{\max }$, and the recently proposed benchmark scenarios, $m_{\mathrm{h}}^{\bmod +}, m_{\mathrm{h}}^{\bmod -}$, light-stop, light-stau, and $\tau$ phobic. In the case of the low- $m_{\mathrm{H}}$ scenario, limits on $\tan \beta$ as a function of the higgsino mass parameter $\mu$ are performed, where $m_{\mathrm{A}}$ is set to a value of $110 \mathrm{GeV}$.

A test of the compatibility of the data to a signal of the three neutral Higgs bosons h, $\mathrm{H}$, and A compared to a SM Higgs boson hypothesis is performed as described in section 7, using the test statistics given by eq. (7.4). The simulation of the SM Higgs boson signal at $125 \mathrm{GeV}$ used in the statistical analysis, is the same as in the dedicated SM Higgs boson search in the $\tau \tau$ decay mode [29], which includes the contributions from gluon fusion, vector boson fusion, and $\mathrm{Z}$ or $\mathrm{W}$ boson and top-quark associated Higgs boson production. The contribution from SM b-quark associated Higgs boson production is expected to be small and is not included in this analysis.

Figure 5 shows the expected and observed exclusion limits at the $95 \%$ CL in the $m_{\mathrm{h}}^{\max }$ scenario and the modified scenarios $m_{\mathrm{h}}^{\bmod +}$ and $m_{\mathrm{h}}^{\bmod -}$. The allowed regions where the mass of the MSSM scalar Higgs boson $\mathrm{h}$ or $\mathrm{H}$ is compatible with the mass of the recently discovered boson of $125 \mathrm{GeV}$ within a range of $\pm 3 \mathrm{GeV}$ are delimited by the hatched areas. Most of the MSSM parameter space is excluded by the Higgs boson mass requirement in the $m_{\mathrm{h}}^{\max }$ scenario, while in the modified scenarios the exclusion is mainly concentrated at low $\tan \beta$ values. Numerical values for the expected and observed exclusion limits for all MSSM benchmark scenarios considered are given in tables 6-12 in appendix A.

It should be noted that due to the interference effects of the bottom and top loops in the MSSM ggh cross section calculation, the direct search is also able to exclude some regions at low $\tan \beta$. The excluded regions at low $\tan \beta$ can be seen better looking at the numerical values given in tables $6-12$ in appendix $\mathrm{A}$.

To allow to compare these results to other extensions of the SM apart from the MSSM, which have been proposed to solve the hierarchy problem, a search for a single resonance $\phi$ with a narrow width compared to the experimental resolution is also performed. In this case, model independent limits on the product of the production cross section times branching fraction to $\tau \tau, \sigma \cdot \mathcal{B}(\phi \rightarrow \tau \tau)$, for gluon fusion and b-quark associated Higgs boson production, as a function of the Higgs boson mass $m_{\phi}$ have been determined. To model the hypothetical signal $\phi$, the same simulation samples as the neutral MSSM Higgs boson search have been used. These results have been obtained using the data with $8 \mathrm{TeV}$ center-of-mass energy only and are shown in figure 7. The expected and observed limits are computed using the test statistics given by eq. (7.1). To extract the limit on the gluon fusion (b-quark associated) Higgs boson production, the rate of the b-quark associated (gluon fusion) Higgs boson production is treated as a nuisance parameter in the fit. For the expected limits, the observed data have been replaced by a representative dataset which not only contains the contribution from background processes but also a SM Higgs boson with a mass of $125 \mathrm{GeV}$. The observed limits are in agreement with the expectation. The results are also summarized in tables 13 and 14 in appendix A. 

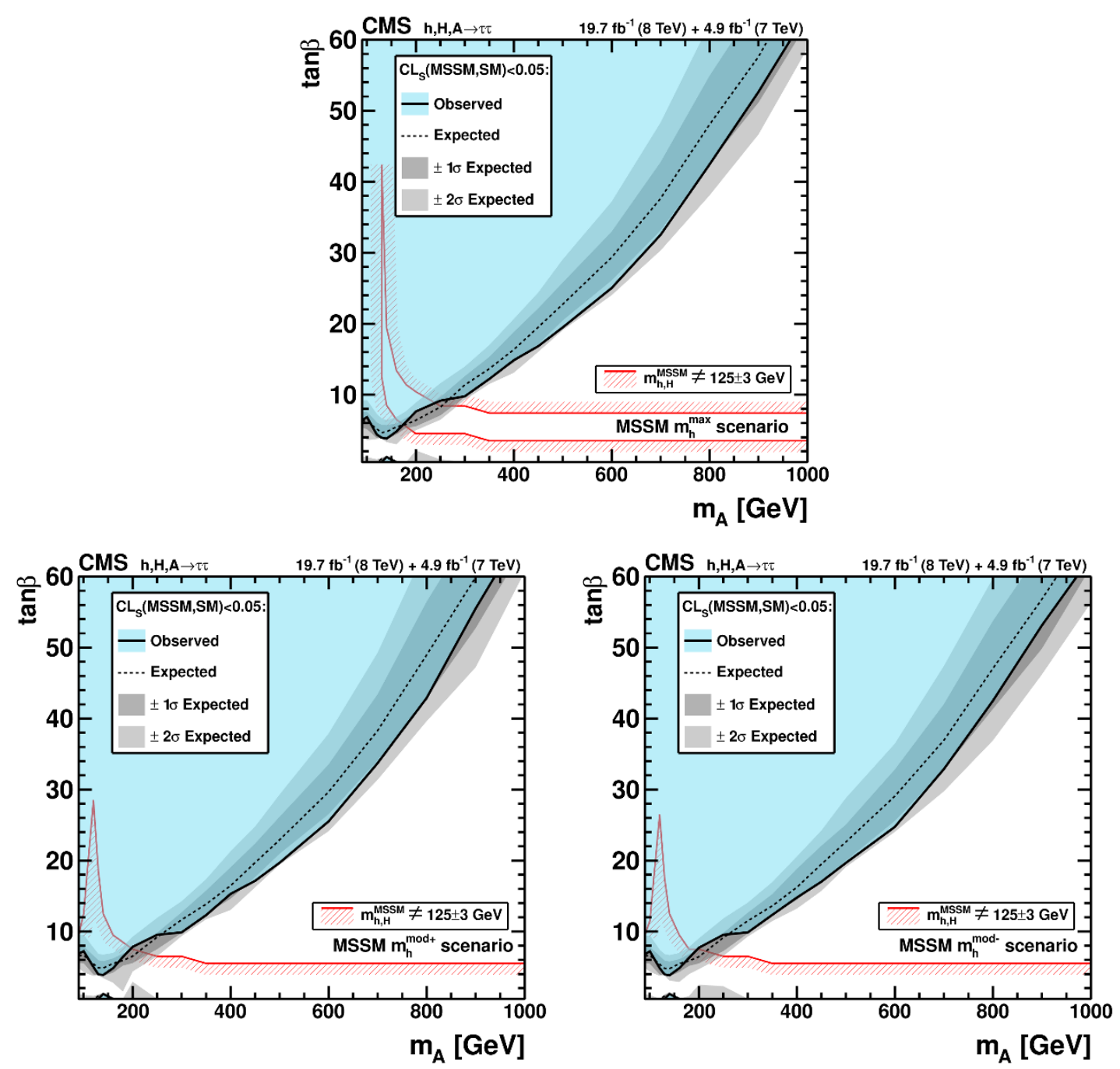

Figure 5. Expected and observed exclusion limits at 95\% CL in the $m_{\mathrm{A}}$-tan $\beta$ parameter space for the MSSM $m_{\mathrm{h}}^{\max }, m_{\mathrm{h}}^{\bmod +}$ and $m_{\mathrm{h}}^{\bmod -}$ benchmark scenarios, are shown as shaded areas. The allowed regions where the mass of the MSSM scalar Higgs boson $\mathrm{h}$ or $\mathrm{H}$ is compatible with the mass of the recently discovered boson of $125 \mathrm{GeV}$ within a range of $\pm 3 \mathrm{GeV}$ are delimited by the hatched areas. A test of the compatibility of the data to a signal of the three neutral Higgs bosons $\mathrm{h}, \mathrm{H}$ and A compared to a SM Higgs boson hypothesis is performed.

Finally, a 2-dimensional $68 \%$ and 95\% CL likelihood scan of the cross section times branching fraction to $\tau \tau$ for gluon fusion and b-quark associated Higgs boson production, $\sigma(\mathrm{bb} \phi) \cdot \mathcal{B}(\phi \rightarrow \tau \tau)$ versus $\sigma(\operatorname{gg} \phi) \cdot \mathcal{B}(\phi \rightarrow \tau \tau)$, has also been performed. The results for different values of the Higgs boson mass $m_{\phi}$ are shown in figure 8 . The best fit value and the expectation from a SM Higgs boson with a mass of $125 \mathrm{GeV}$ is also shown. The result from the likelihood scan for $m_{\phi}=125 \mathrm{GeV}$ is compatible with the expectation from a SM Higgs boson. 

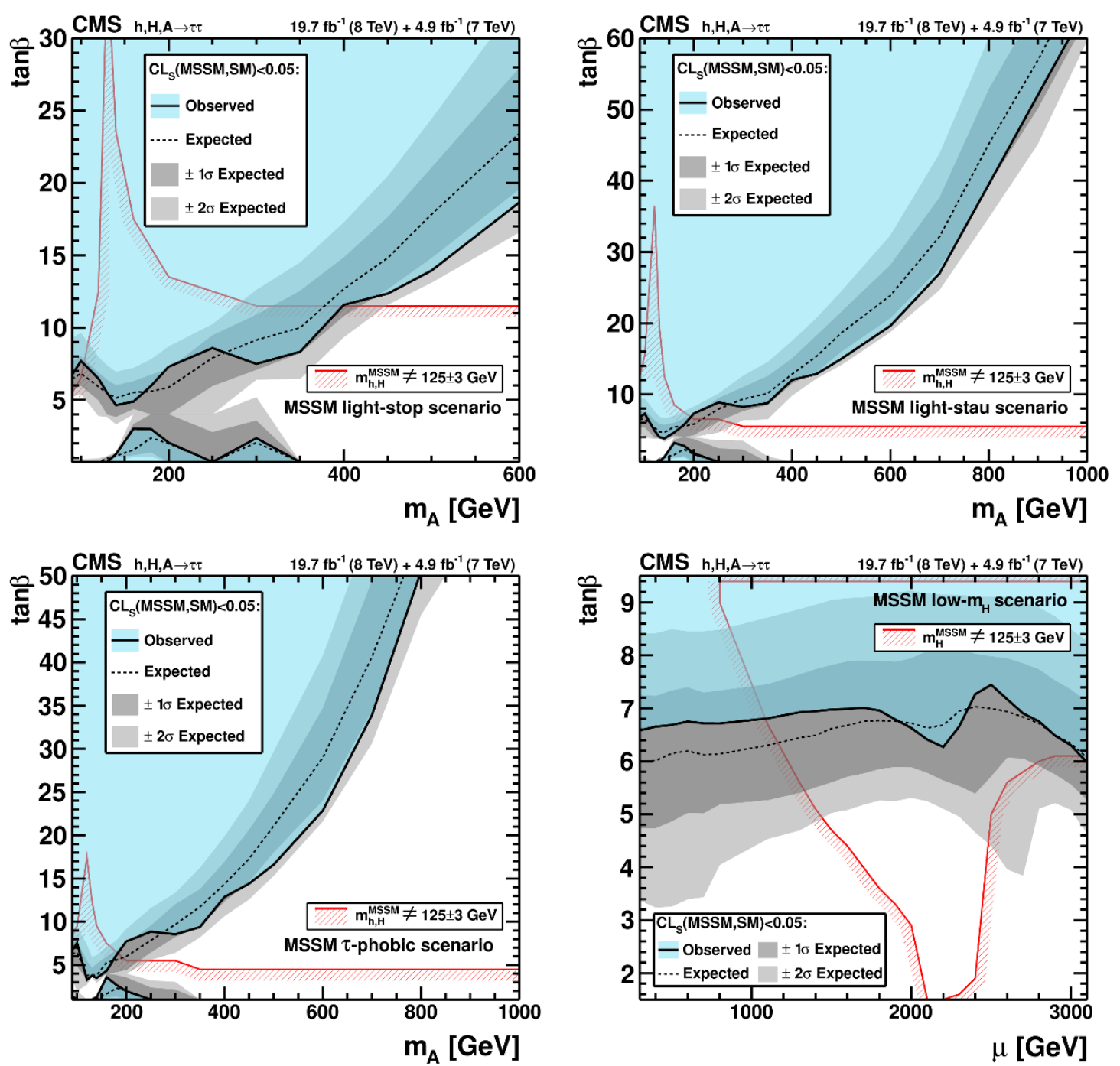

Figure 6. Expected and observed exclusion limits at $95 \% \mathrm{CL}$ in the $m_{\mathrm{A}}$-tan $\beta$ parameter space for the MSSM light-stop, light-stau, and $\tau$-phobic benchmark scenarios. In the MSSM low- $m_{\mathrm{H}}$ benchmark scenario the limits in the $\mu$ - $\tan \beta$ parameter space are shown. Other details are as in figure 5 .

\section{Summary}

A search for neutral Higgs bosons in the minimal supersymmetric extension of the standard model (MSSM) decaying to tau-lepton pairs has been performed using events recorded by the CMS experiment at the LHC. The dataset corresponds to an integrated luminosity of $24.6 \mathrm{fb}^{-1}$, with $4.9 \mathrm{fb}^{-1}$ at $7 \mathrm{TeV}$ and $19.7 \mathrm{fb}^{-1}$ at $8 \mathrm{TeV}$. Five different $\tau \tau$ signatures are studied, $\mathrm{e} \tau_{\mathrm{h}}, \mu \tau_{\mathrm{h}}, \mathrm{e} \mu, \mu \mu$, and $\tau_{\mathrm{h}} \tau_{\mathrm{h}}$, where $\tau_{\mathrm{h}}$ denotes a hadronically decaying tau lepton. To enhance the sensitivity to neutral MSSM Higgs bosons, the search includes the case where the Higgs boson is produced in association with a b-quark jet. No excess is observed in the tau-lepton-pair invariant mass spectrum. Exclusion limits are presented in the MSSM 

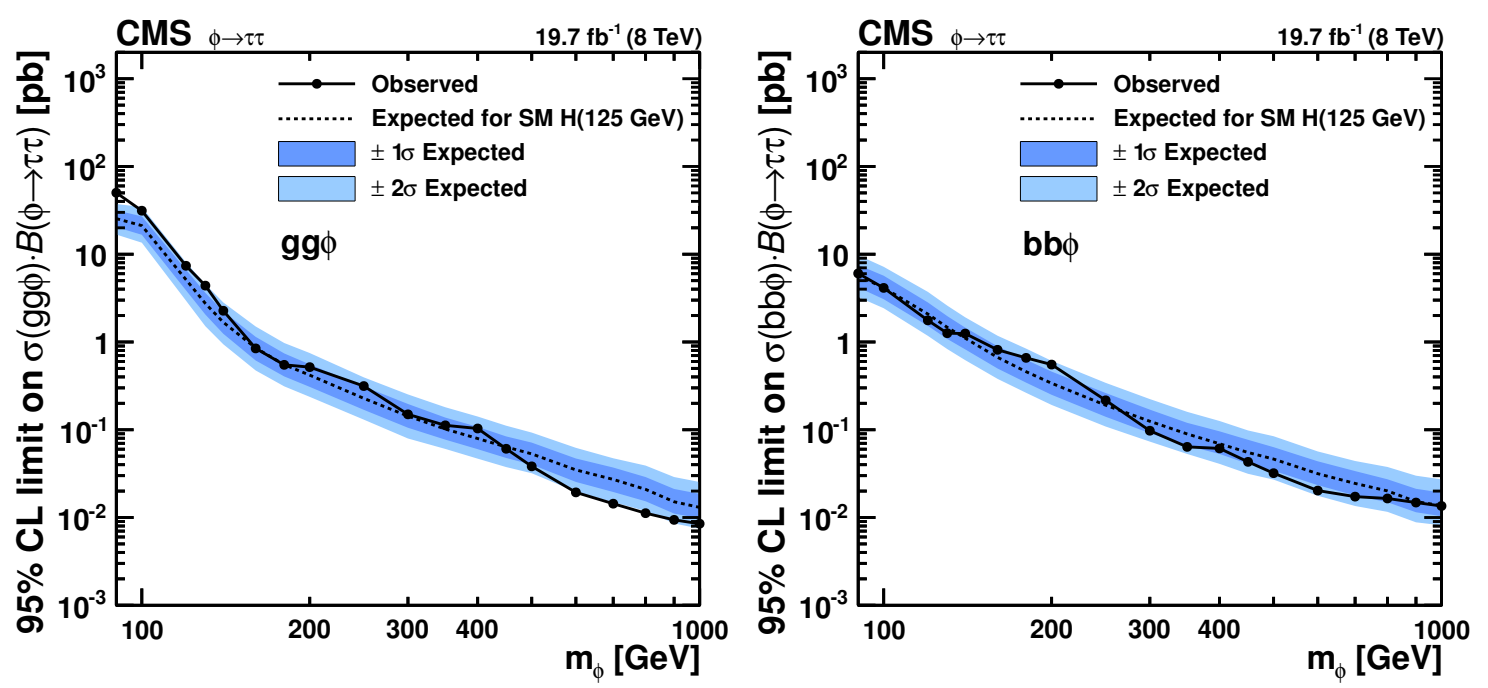

Figure 7. Upper limit at $95 \% \mathrm{CL}$ on $\sigma(\operatorname{gg} \phi) \cdot \mathcal{B}(\phi \rightarrow \tau \tau)$ (left) and $\sigma(\mathrm{bb} \phi) \cdot \mathcal{B}(\phi \rightarrow \tau \tau)$ (right) at $8 \mathrm{TeV}$ center-of-mass energy as a function of $m_{\phi}$, where $\phi$ denotes a generic Higgs-like state. The expected and observed limits are computed using the test statistics given by eq. (7.1). For the expected limits, the observed data have been replaced by a representative dataset which not only contains the contribution from background processes but also a SM Higgs boson with a mass of $125 \mathrm{GeV}$.

parameter space for different MSSM benchmark scenarios, $m_{\mathrm{h}}^{\max }, m_{\mathrm{h}}^{\bmod +}, m_{\mathrm{h}}^{\bmod -}$, lightstop, light-stau, $\tau$-phobic and low- $m_{\mathrm{H}}$. Model independent upper limits on the Higgs boson production cross section times branching fraction for gluon fusion and b-quark associated production are also given.

\section{Acknowledgments}

We congratulate our colleagues in the CERN accelerator departments for the excellent performance of the LHC and thank the technical and administrative staffs at CERN and at other CMS institutes for their contributions to the success of the CMS effort. In addition, we gratefully acknowledge the computing centres and personnel of the Worldwide LHC Computing Grid for delivering so effectively the computing infrastructure essential to our analyses. Finally, we acknowledge the enduring support for the construction and operation of the LHC and the CMS detector provided by the following funding agencies: the Austrian Federal Ministry of Science, Research and Economy and the Austrian Science Fund; the Belgian Fonds de la Recherche Scientifique, and Fonds voor Wetenschappelijk Onderzoek; the Brazilian Funding Agencies (CNPq, CAPES, FAPERJ, and FAPESP); the Bulgarian Ministry of Education and Science; CERN; the Chinese Academy of Sciences, Ministry of Science and Technology, and National Natural Science Foundation of China; the Colombian Funding Agency (COLCIENCIAS); the Croatian Ministry of Science, Education and Sport, and the Croatian Science Foundation; the Research Promotion Foundation, Cyprus; the Ministry of Education and Research, Estonian Research Council via IUT23-4 and IUT23- 

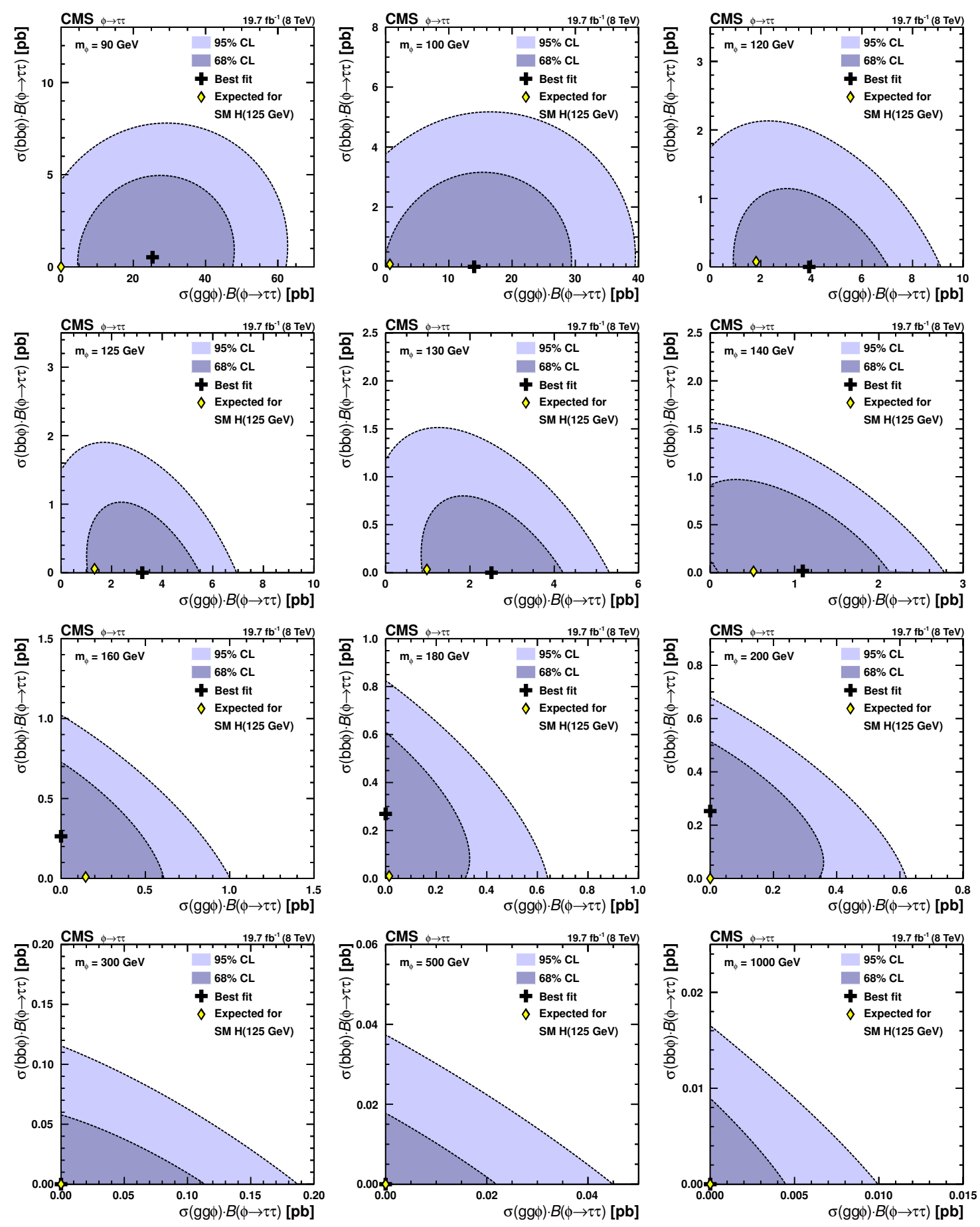

Figure 8. Likelihood contours of $\sigma(\mathrm{bb} \phi) \cdot \mathcal{B}(\phi \rightarrow \tau \tau)$ versus $\sigma(\operatorname{gg} \phi) \cdot \mathcal{B}(\phi \rightarrow \tau \tau)$ at $8 \mathrm{TeV}$ centerof-mass energy for different values of the Higgs boson mass $m_{\phi}$. The best fit value (cross) and the expectation from a SM Higgs boson with a mass of $125 \mathrm{GeV}$ (diamond) is also shown. In the case of $m_{\phi}=300,500$ and $1000 \mathrm{GeV}$, the best fit value and the expectation from a SM Higgs boson at $125 \mathrm{GeV}$ are both at $(0,0)$. 
6 and European Regional Development Fund, Estonia; the Academy of Finland, Finnish Ministry of Education and Culture, and Helsinki Institute of Physics; the Institut National de Physique Nucléaire et de Physique des Particules / CNRS, and Commissariat à l'Énergie Atomique et aux Énergies Alternatives / CEA, France; the Bundesministerium für Bildung und Forschung, Deutsche Forschungsgemeinschaft, and Helmholtz-Gemeinschaft Deutscher Forschungszentren, Germany; the General Secretariat for Research and Technology, Greece; the National Scientific Research Foundation, and National Innovation Office, Hungary; the Department of Atomic Energy and the Department of Science and Technology, India; the Institute for Studies in Theoretical Physics and Mathematics, Iran; the Science Foundation, Ireland; the Istituto Nazionale di Fisica Nucleare, Italy; the Korean Ministry of Education, Science and Technology and the World Class University program of NRF, Republic of Korea; the Lithuanian Academy of Sciences; the Ministry of Education, and University of Malaya (Malaysia); the Mexican Funding Agencies (CINVESTAV, CONACYT, SEP, and UASLP-FAI); the Ministry of Business, Innovation and Employment, New Zealand; the Pakistan Atomic Energy Commission; the Ministry of Science and Higher Education and the National Science Centre, Poland; the Fundação para a Ciência e a Tecnologia, Portugal; JINR, Dubna; the Ministry of Education and Science of the Russian Federation, the Federal Agency of Atomic Energy of the Russian Federation, Russian Academy of Sciences, and the Russian Foundation for Basic Research; the Ministry of Education, Science and Technological Development of Serbia; the Secretaría de Estado de Investigación, Desarrollo e Innovación and Programa Consolider-Ingenio 2010, Spain; the Swiss Funding Agencies (ETH Board, ETH Zurich, PSI, SNF, UniZH, Canton Zurich, and SER); the Ministry of Science and Technology, Taipei; the Thailand Center of Excellence in Physics, the Institute for the Promotion of Teaching Science and Technology of Thailand, Special Task Force for Activating Research and the National Science and Technology Development Agency of Thailand; the Scientific and Technical Research Council of Turkey, and Turkish Atomic Energy Authority; the National Academy of Sciences of Ukraine, and State Fund for Fundamental Researches, Ukraine; the Science and Technology Facilities Council, U.K.; the US Department of Energy, and the US National Science Foundation.

Individuals have received support from the Marie-Curie programme and the European Research Council and EPLANET (European Union); the Leventis Foundation; the A. P. Sloan Foundation; the Alexander von Humboldt Foundation; the Belgian Federal Science Policy Office; the Fonds pour la Formation à la Recherche dans l'Industrie et dans l'Agriculture (FRIA-Belgium); the Agentschap voor Innovatie door Wetenschap en Technologie (IWT-Belgium); the Ministry of Education, Youth and Sports (MEYS) of the Czech Republic; the Council of Science and Industrial Research, India; the HOMING PLUS programme of Foundation for Polish Science, cofinanced from European Union, Regional Development Fund; the Compagnia di San Paolo (Torino); the Consorzio per la Fisica (Trieste); MIUR project 20108T4XTM (Italy); the Thalis and Aristeia programmes cofinanced by EU-ESF and the Greek NSRF; and the National Priorities Research Program by Qatar National Research Fund. 


\section{A Exclusion limits}

The $95 \%$ CL exclusion limits in the $\tan \beta-m_{\mathrm{A}}$ or $\tan \beta-\mu$ parameter space for different MSSM benchmark scenarios are given in tables $6-12$.

Model independent limits on $\sigma \cdot \mathcal{B}(\phi \rightarrow \tau \tau)$ for gluon fusion and b-quark associated Higgs boson production as a function of the Higgs boson mass $m_{\phi}$ are given in tables 13 and 14, for $8 \mathrm{TeV}$ center-of-mass energy only.

\begin{tabular}{|c|ccccc|c|}
\hline \multirow{2}{*}{$m_{\mathrm{A}}[\mathrm{GeV}]$} & \multicolumn{5}{c|}{ Neutral MSSM Higgs: $m_{\mathrm{h}}^{\max }$ scenario } \\
\cline { 2 - 6 } & \multicolumn{5}{c}{ Expected tan $\beta$ limit } & Observed \\
\hline \multirow{2}{*}{90} & $>4.3$ & $>5.3$ & $>6.3$ & $>7.6$ & $>8.3$ & $>6.4$ \\
& $<1.3$ & & & & & \\
100 & $>3.6$ & $>5.1$ & $>6.3$ & $>7.6$ & $>9.0$ & $>6.8$ \\
& $<1.2$ & & & & & \\
120 & $>3.8$ & $>3.9$ & $>5.2$ & $>6.1$ & $>6.6$ & $>4.4$ \\
& $<0.9$ & & & & & \\
130 & $>3.8$ & $>3.9$ & $>4.7$ & $>5.7$ & $>5.5$ & $>3.9$ \\
& $<1.1$ & $<1.1$ & $<0.9$ & & & \\
140 & $>3.3$ & $>3.7$ & $>4.8$ & $>5.7$ & $>6.2$ & $>3.8$ \\
160 & $>1.4$ & $<1.3$ & $<1.1$ & $<1.0$ & & $<1.2$ \\
180 & $>3.0$ & $>4.4$ & $>5.5$ & $>5.9$ & $>7.9$ & $>4.8$ \\
200 & $>4.4$ & $>5.3$ & $>5.8$ & $>6.7$ & $>7.2$ & $>6.2$ \\
& $>5.0$ & $>5.6$ & $>6.4$ & $>7.4$ & $>7.8$ & $>7.6$ \\
250 & $>2.1$ & & & & & \\
300 & $>6.5$ & $>7.1$ & $>8.3$ & $>9.6$ & $>10.0$ & $>9.2$ \\
350 & $>0.8$ & & & & & \\
400 & $>9.3$ & $>9.7$ & $>11.4$ & $>12.6$ & $>13.5$ & $>9.8$ \\
450 & $>13.1$ & $>14.5$ & $>16.3$ & $>18.8$ & $>19.6$ & $>14.9$ \\
500 & $>16.0$ & $>17.1$ & $>19.5$ & $>22.1$ & $>23.0$ & $>16.8$ \\
600 & $>24.0$ & $>26.1$ & $>29.3$ & $>33.0$ & $>34.7$ & $>25.0$ \\
700 & $>30.3$ & $>33.2$ & $>37.7$ & $>42.7$ & $>45.0$ & $>32.5$ \\
800 & $>38.1$ & $>42.6$ & $>48.1$ & $>54.9$ & - & $>42.4$ \\
900 & $>46.7$ & $>51.2$ & $>57.6$ & - & - & $>52.7$ \\
1000 & $>58.8$ & - & - & - & - & - \\
\hline
\end{tabular}

Table 6. Expected and observed 95\% CL limits for $\tan \beta$ as a function of $m_{\mathrm{A}}$, for the MSSM search in the $m_{\mathrm{h}}^{\max }$ benchmark scenario. For the statistical procedure a test of the compatibility of the data to a signal of the three neutral Higgs bosons h, H and A compared to a SM Higgs boson hypothesis is performed. 


\begin{tabular}{|c|c|c|c|c|c|c|}
\hline \multirow[b]{3}{*}{$m_{\mathrm{A}}[\mathrm{GeV}]$} & \multicolumn{6}{|c|}{ Neutral MSSM Higgs: $m_{\mathrm{h}}^{\bmod +}$ scenario } \\
\hline & \multicolumn{5}{|c|}{ Expected $\tan \beta$ limit } & \multirow{2}{*}{$\begin{array}{l}\text { Observed } \\
\tan \beta \text { limit }\end{array}$} \\
\hline & $-2 \sigma$ & $-1 \sigma$ & Median & $+1 \sigma$ & $+2 \sigma$ & \\
\hline \multirow{2}{*}{90} & $>4.9$ & $>5.6$ & $>6.6$ & $>7.8$ & $>8.3$ & $>6.9$ \\
\hline & $<1.2$ & & & & & \\
\hline \multirow{2}{*}{100} & $>4.3$ & $>5.5$ & $>6.7$ & $>7.9$ & $>9.0$ & $>7.2$ \\
\hline & $<1.1$ & & & & & \\
\hline \multirow{2}{*}{120} & $>3.8$ & $>4.0$ & $>5.4$ & $>6.6$ & $>7.1$ & $>5.0$ \\
\hline & $<0.9$ & & & & & \\
\hline \multirow{2}{*}{130} & $>3.8$ & $>3.9$ & $>4.9$ & $>5.9$ & $>6.0$ & $>4.0$ \\
\hline & $<1.1$ & $<1.1$ & $<0.9$ & & & \\
\hline \multirow{2}{*}{140} & $>3.4$ & $>3.7$ & $>4.8$ & $>5.8$ & $>6.3$ & $>3.9$ \\
\hline & $<1.4$ & $<1.2$ & $<1.1$ & $<1.0$ & & $<1.2$ \\
\hline 160 & $>2.8$ & $>4.2$ & $>5.5$ & $>6.2$ & $>8.2$ & $>4.8$ \\
\hline 180 & $>1.6$ & $>5.2$ & $>5.9$ & $>6.9$ & $>10.2$ & $>6.4$ \\
\hline \multirow{2}{*}{200} & $>4.4$ & $>5.6$ & $>6.5$ & $>7.6$ & $>8.7$ & $>7.8$ \\
\hline & $<2.9$ & & & & & \\
\hline 250 & $>6.5$ & $>7.5$ & $>9.2$ & $>9.8$ & $>11.9$ & $>9.5$ \\
\hline 300 & $>9.4$ & $>9.8$ & $>11.7$ & $>12.7$ & $>13.9$ & $>9.9$ \\
\hline 350 & $>11.7$ & $>12.5$ & $>13.9$ & $>15.8$ & $>16.0$ & $>12.3$ \\
\hline 400 & $>13.0$ & $>14.6$ & $>16.5$ & $>19.0$ & $>19.9$ & $>15.3$ \\
\hline 450 & $>16.2$ & $>17.7$ & $>19.7$ & $>22.4$ & $>23.2$ & $>17.1$ \\
\hline 500 & $>19.5$ & $>21.0$ & $>22.9$ & $>26.1$ & $>26.4$ & $>19.7$ \\
\hline 600 & $>24.1$ & $>26.5$ & $>29.7$ & $>33.5$ & $>35.2$ & $>25.5$ \\
\hline 700 & $>31.4$ & $>34.1$ & $>38.3$ & $>43.4$ & $>45.2$ & $>33.7$ \\
\hline 800 & $>39.6$ & $>43.0$ & $>49.0$ & $>56.4$ & - & $>42.9$ \\
\hline 900 & $>47.2$ & $>52.8$ & $>59.9$ & - & - & $>55.5$ \\
\hline 1000 & - & - & - & - & - & - \\
\hline
\end{tabular}

Table 7. Expected and observed 95\% CL limits for $\tan \beta$ as a function of $m_{\mathrm{A}}$, for the MSSM search in the $m_{\mathrm{h}}^{\bmod +}$ benchmark scenario. For the statistical procedure a test of the compatibility of the data to a signal of the three neutral Higgs bosons h, H and A compared to a SM Higgs boson hypothesis is performed. 


\begin{tabular}{|c|c|c|c|c|c|c|}
\hline \multirow[b]{3}{*}{$m_{\mathrm{A}}[\mathrm{GeV}]$} & \multicolumn{6}{|c|}{ Neutral MSSM Higgs: $m_{\mathrm{h}}^{\text {mod- }}$ scenario } \\
\hline & \multicolumn{5}{|c|}{ Expected $\tan \beta$ limit } & \multirow{2}{*}{$\begin{array}{c}\text { Observed } \\
\tan \beta \text { limit }\end{array}$} \\
\hline & $-2 \sigma$ & $-1 \sigma$ & Median & $+1 \sigma$ & $+2 \sigma$ & \\
\hline \multirow{2}{*}{90} & $>4.9$ & $>5.6$ & $>6.5$ & $>7.7$ & $>8.2$ & $>6.8$ \\
\hline & $<1.1$ & & & & & \\
\hline \multirow{2}{*}{100} & $>4.4$ & $>5.5$ & $>6.6$ & $>7.8$ & $>8.9$ & $>7.2$ \\
\hline & $<1.0$ & & & & & \\
\hline 120 & $>3.8$ & $>4.0$ & $>5.4$ & $>6.5$ & $>7.0$ & $>4.8$ \\
\hline \multirow{2}{*}{130} & $>3.8$ & $>3.9$ & $>4.8$ & $>5.9$ & $>5.8$ & $>4.0$ \\
\hline & $<1.1$ & $<1.1$ & $<0.9$ & & & \\
\hline \multirow{2}{*}{140} & $>3.1$ & $>3.7$ & $>4.8$ & $>5.8$ & $>6.4$ & $>3.8$ \\
\hline & $<1.5$ & $<1.2$ & $<1.1$ & $<1.0$ & & $<1.2$ \\
\hline 160 & $>2.9$ & $>4.3$ & $>5.5$ & $>6.1$ & $>8.0$ & $>4.8$ \\
\hline \multirow{2}{*}{180} & $>4.0$ & $>5.2$ & $>5.9$ & $>6.8$ & $>7.7$ & $>6.3$ \\
\hline & $<0.9$ & & & & & \\
\hline \multirow{2}{*}{200} & $>4.7$ & $>5.5$ & $>6.5$ & $>7.5$ & $>8.2$ & $>7.7$ \\
\hline & $<2.5$ & & & & & \\
\hline \multirow{2}{*}{250} & $>6.6$ & $>7.3$ & $>9.1$ & $>9.8$ & $>11.7$ & $>9.5$ \\
\hline & $<2.3$ & & & & & \\
\hline 300 & $>9.3$ & $>9.8$ & $>11.5$ & $>12.6$ & $>13.7$ & $>9.9$ \\
\hline 350 & $>11.5$ & $>12.4$ & $>13.6$ & $>15.3$ & $>15.7$ & $>12.3$ \\
\hline 400 & $>13.2$ & $>14.5$ & $>16.2$ & $>18.8$ & $>19.2$ & $>14.8$ \\
\hline 450 & $>15.7$ & $>17.2$ & $>19.5$ & $>22.1$ & $>23.2$ & $>17.0$ \\
\hline 500 & $>19.1$ & $>20.0$ & $>22.6$ & $>25.0$ & $>26.1$ & $>19.7$ \\
\hline 600 & $>24.0$ & $>25.6$ & $>29.1$ & $>32.8$ & $>34.1$ & $>24.7$ \\
\hline 700 & $>29.8$ & $>32.8$ & $>36.9$ & $>42.0$ & $>44.1$ & $>32.9$ \\
\hline 800 & $>36.9$ & $>41.4$ & $>47.1$ & $>53.7$ & $>57.2$ & $>42.5$ \\
\hline 900 & $>46.2$ & $>49.9$ & $>56.8$ & - & - & $>53.1$ \\
\hline 1000 & $>56.3$ & - & - & - & - & - \\
\hline
\end{tabular}

Table 8. Expected and observed 95\% CL limits for $\tan \beta$ as a function of $m_{\mathrm{A}}$, for the MSSM search in the $m_{\mathrm{h}}^{\bmod -}$ benchmark scenario. For the statistical procedure a test of the compatibility of the data to a signal of the three neutral Higgs bosons h, H and A compared to a SM Higgs boson hypothesis is performed. 


\begin{tabular}{|c|c|c|c|c|c|c|}
\hline \multirow[b]{3}{*}{$m_{\mathrm{A}}[\mathrm{GeV}]$} & \multicolumn{6}{|c|}{ Neutral MSSM Higgs: light-stop scenario } \\
\hline & \multicolumn{5}{|c|}{ Expected $\tan \beta$ limit } & \multirow{2}{*}{$\begin{array}{l}\text { Observed } \\
\tan \beta \text { limit }\end{array}$} \\
\hline & $-2 \sigma$ & $-1 \sigma$ & Median & $+1 \sigma$ & $+2 \sigma$ & \\
\hline \multirow{2}{*}{90} & $>5.2$ & $>5.7$ & $>6.4$ & $>7.7$ & $>7.7$ & $>6.7$ \\
\hline & $<1.2$ & & & & & \\
\hline \multirow{2}{*}{100} & $>5.4$ & $>6.0$ & $>6.8$ & $>8.0$ & $>8.3$ & $>7.7$ \\
\hline & $<0.9$ & & & & & \\
\hline \multirow{2}{*}{120} & $>3.9$ & $>5.0$ & $>5.9$ & $>6.9$ & $>7.9$ & $>6.5$ \\
\hline & $<0.9$ & & & & & \\
\hline \multirow{2}{*}{130} & $>3.8$ & $>4.0$ & $>5.4$ & $>6.4$ & $>7.1$ & $>5.5$ \\
\hline & $<1.1$ & $<1.1$ & $<0.9$ & & & \\
\hline \multirow{2}{*}{140} & $>3.3$ & $>3.8$ & $>5.1$ & $>6.0$ & $>7.0$ & $>4.6$ \\
\hline & $<1.4$ & $<1.2$ & $<1.1$ & $<1.0$ & & $<1.2$ \\
\hline \multirow{2}{*}{160} & $>3.3$ & $>4.4$ & $>5.5$ & $>6.3$ & $>7.2$ & $>4.9$ \\
\hline & $<1.4$ & $<3.3$ & $<1.5$ & $<1.2$ & $<1.1$ & $<3.0$ \\
\hline \multirow{2}{*}{180} & $>3.3$ & $>4.4$ & $>5.6$ & $>6.7$ & $>7.8$ & $>6.0$ \\
\hline & $<1.4$ & $<3.3$ & $<2.4$ & $<1.4$ & $<1.1$ & $<3.0$ \\
\hline \multirow{2}{*}{200} & $>3.3$ & $>4.4$ & $>5.9$ & $>7.1$ & $>7.7$ & $>7.3$ \\
\hline & $<1.4$ & $<3.3$ & $<2.0$ & & & $<2.0$ \\
\hline \multirow{2}{*}{250} & $>3.3$ & $>6.0$ & $>7.9$ & $>9.5$ & $>11.8$ & $>8.6$ \\
\hline & $<1.4$ & $<2.8$ & & & & \\
\hline \multirow{2}{*}{300} & $>6.4$ & $>7.1$ & $>9.1$ & $>10.9$ & $>11.9$ & $>7.5$ \\
\hline & $<5.2$ & $<3.6$ & $<2.0$ & & & $<2.3$ \\
\hline 350 & $>6.4$ & $>8.2$ & $>10.0$ & $>12.5$ & $>13.5$ & $>8.3$ \\
\hline 400 & $>9.3$ & $>10.8$ & $>12.7$ & $>14.9$ & $>16.0$ & $>11.6$ \\
\hline 450 & $>11.6$ & $>12.7$ & $>14.8$ & $>17.6$ & $>18.1$ & $>12.3$ \\
\hline 500 & $>13.1$ & $>14.8$ & $>17.8$ & $>21.1$ & $>22.6$ & $>13.9$ \\
\hline 600 & $>16.6$ & $>19.6$ & $>23.4$ & $>28.0$ & $>30.3$ & $>18.7$ \\
\hline
\end{tabular}

Table 9. Expected and observed 95\% CL limits for $\tan \beta$ as a function of $m_{\mathrm{A}}$, for the MSSM search in the light-stop benchmark scenario. For the statistical procedure a test of the compatibility of the data to a signal of the three neutral Higgs bosons h, H and A compared to a SM Higgs boson hypothesis is performed. 


\begin{tabular}{|c|c|c|c|c|c|c|}
\hline \multirow[b]{3}{*}{$m_{\mathrm{A}}[\mathrm{GeV}]$} & \multicolumn{6}{|c|}{ Neutral MSSM Higgs: light-stau scenario } \\
\hline & \multicolumn{5}{|c|}{ Expected $\tan \beta$ limit } & \multirow{2}{*}{$\begin{array}{c}\text { Observed } \\
\tan \beta \text { limit }\end{array}$} \\
\hline & $-2 \sigma$ & $-1 \sigma$ & Median & $+1 \sigma$ & $+2 \sigma$ & \\
\hline \multirow{2}{*}{90} & $>4.5$ & $>5.4$ & $>6.5$ & $>7.7$ & $>8.4$ & $>6.7$ \\
\hline & $<1.1$ & & & & & \\
\hline \multirow{2}{*}{100} & $>4.0$ & $>5.4$ & $>6.5$ & $>7.8$ & $>9.0$ & $>7.3$ \\
\hline & $<1.1$ & & & & & \\
\hline 120 & $>3.7$ & $>3.9$ & $>5.3$ & $>6.4$ & $>6.9$ & $>4.9$ \\
\hline \multirow{2}{*}{130} & $>3.8$ & $>3.9$ & $>4.7$ & $>5.8$ & $>5.7$ & $>4.0$ \\
\hline & $<1.1$ & $<1.1$ & $<0.9$ & & & \\
\hline \multirow{2}{*}{140} & $>3.3$ & $>3.7$ & $>4.7$ & $>5.7$ & $>6.0$ & $>3.8$ \\
\hline & $<1.4$ & $<1.2$ & $<1.1$ & $<1.0$ & & $<1.2$ \\
\hline \multirow{2}{*}{160} & $>3.3$ & $>4.2$ & $>5.4$ & $>6.0$ & $>7.0$ & $>4.6$ \\
\hline & $<1.4$ & $<3.6$ & $<1.5$ & $<1.2$ & $<1.1$ & $<3.2$ \\
\hline \multirow{2}{*}{180} & $>3.3$ & $>4.2$ & $>5.5$ & $>6.6$ & $>7.6$ & $>5.7$ \\
\hline & $<1.4$ & $<3.6$ & $<2.3$ & $<1.3$ & $<1.1$ & $<2.8$ \\
\hline \multirow{2}{*}{200} & $>3.3$ & $>4.3$ & $>5.8$ & $>7.0$ & $>7.6$ & $>7.3$ \\
\hline & $<1.4$ & $<3.7$ & $<1.6$ & & & $<1.7$ \\
\hline \multirow{2}{*}{250} & $>4.6$ & $>6.2$ & $>7.9$ & $>9.5$ & $>11.2$ & $>8.9$ \\
\hline & $<3.5$ & $<2.4$ & & & & \\
\hline \multirow{2}{*}{300} & $>6.4$ & $>7.7$ & $>9.3$ & $>11.0$ & $>12.3$ & $>8.2$ \\
\hline & $<3.5$ & $<2.2$ & & & & \\
\hline \multirow{2}{*}{350} & $>6.5$ & $>8.5$ & $>10.1$ & $>12.5$ & $>13.8$ & $>8.8$ \\
\hline & $<1.3$ & & & & & \\
\hline 400 & $>9.8$ & $>11.6$ & $>12.8$ & $>15.0$ & $>15.8$ & $>12.0$ \\
\hline 450 & $>12.2$ & $>13.2$ & $>15.4$ & $>18.3$ & $>18.5$ & $>12.9$ \\
\hline 500 & $>14.1$ & $>16.0$ & $>18.6$ & $>21.6$ & $>23.1$ & $>15.0$ \\
\hline 600 & $>18.8$ & $>20.7$ & $>23.9$ & $>28.2$ & $>29.0$ & $>19.6$ \\
\hline 700 & $>24.7$ & $>27.7$ & $>32.2$ & $>37.6$ & $>39.6$ & $>27.0$ \\
\hline 800 & $>34.8$ & $>39.4$ & $>45.1$ & $>52.8$ & - & $>39.5$ \\
\hline 900 & $>45.2$ & $>50.2$ & $>56.9$ & - & - & $>52.0$ \\
\hline 1000 & $>59.6$ & - & - & - & - & - \\
\hline
\end{tabular}

Table 10. Expected and observed 95\% CL limits for $\tan \beta$ as a function of $m_{\mathrm{A}}$, for the MSSM search in the light-stau benchmark scenario. For the statistical procedure a test of the compatibility of the data to a signal of the three neutral Higgs bosons h, H and A compared to a SM Higgs boson hypothesis is performed. 


\begin{tabular}{|c|c|c|c|c|c|c|}
\hline \multirow[b]{3}{*}{$m_{\mathrm{A}}[\mathrm{GeV}]$} & \multicolumn{6}{|c|}{ Neutral MSSM Higgs: $\tau$-phobic scenario } \\
\hline & \multicolumn{5}{|c|}{ Expected $\tan \beta$ limit } & \multirow{2}{*}{$\begin{array}{c}\text { Observed } \\
\tan \beta \text { limit }\end{array}$} \\
\hline & $-2 \sigma$ & $-1 \sigma$ & Median & $+1 \sigma$ & $+2 \sigma$ & \\
\hline \multirow{2}{*}{90} & $>3.3$ & $>5.4$ & $>6.3$ & $>7.5$ & $>9.2$ & $>6.7$ \\
\hline & $<1.8$ & $<1.4$ & $<1.2$ & $<1.1$ & $<1.1$ & $<1.4$ \\
\hline \multirow{2}{*}{100} & $>3.3$ & $>4.8$ & $>6.5$ & $>7.8$ & $>10.9$ & $>7.5$ \\
\hline & $<1.8$ & & & & & \\
\hline 120 & $>2.7$ & $>3.3$ & $>3.8$ & $>5.5$ & $>4.9$ & $>3.3$ \\
\hline \multirow{2}{*}{130} & $>3.6$ & $>3.7$ & $>3.9$ & $>4.7$ & $>4.2$ & $>3.8$ \\
\hline & $<1.2$ & $<1.1$ & & & & \\
\hline \multirow{2}{*}{140} & $>2.9$ & $>3.4$ & $>3.8$ & $>5.1$ & $>4.7$ & $>3.5$ \\
\hline & $<1.5$ & $<1.3$ & $<1.1$ & $<1.0$ & & $<1.3$ \\
\hline \multirow{2}{*}{160} & $>2.9$ & $>3.4$ & $>5.3$ & $>6.0$ & $>7.1$ & $>4.3$ \\
\hline & $<1.5$ & $<1.3$ & $<1.7$ & $<1.2$ & $<1.1$ & $<3.6$ \\
\hline \multirow{2}{*}{180} & $>2.9$ & $>4.2$ & $>5.6$ & $>6.7$ & $>7.8$ & $>6.1$ \\
\hline & $<1.5$ & $<3.8$ & $<2.3$ & $<1.4$ & $<1.1$ & $<2.7$ \\
\hline \multirow{2}{*}{200} & $>2.9$ & $>4.4$ & $>6.0$ & $>7.4$ & $>8.0$ & $>7.7$ \\
\hline & $<1.5$ & $<3.6$ & $<2.0$ & & & $<2.0$ \\
\hline \multirow{2}{*}{250} & $>5.6$ & $>6.4$ & $>7.8$ & $>9.6$ & $>10.1$ & $>8.8$ \\
\hline & $<3.2$ & $<2.4$ & & & & \\
\hline \multirow{2}{*}{300} & $>6.4$ & $>8.3$ & $>9.7$ & $>12.0$ & $>13.0$ & $>8.6$ \\
\hline & $<3.0$ & $<2.2$ & & & & \\
\hline 350 & $>8.2$ & $>9.4$ & $>11.7$ & $>13.7$ & $>15.3$ & $>9.4$ \\
\hline 400 & $>10.7$ & $>12.3$ & $>14.4$ & $>16.9$ & $>18.1$ & $>12.9$ \\
\hline 450 & $>12.6$ & $>14.6$ & $>17.3$ & $>20.6$ & $>22.0$ & $>14.4$ \\
\hline 500 & $>15.3$ & $>18.0$ & $>21.1$ & $>24.8$ & $>26.8$ & $>16.6$ \\
\hline 600 & $>21.6$ & $>24.0$ & $>29.0$ & $>34.7$ & $>36.3$ & $>22.9$ \\
\hline 700 & $>30.6$ & $>34.5$ & $>40.6$ & $>49.5$ & - & $>33.9$ \\
\hline 800 & $>45.5$ & - & - & - & - & - \\
\hline 900 & - & - & - & - & - & - \\
\hline 1000 & - & - & - & - & - & - \\
\hline
\end{tabular}

Table 11. Expected and observed 95\% CL limits for $\tan \beta$ as a function of $m_{\mathrm{A}}$, for the MSSM search in the $\tau$-phobic benchmark scenario. For the statistical procedure a test of the compatibility of the data to a signal of the three neutral Higgs bosons h, H and A compared to a SM Higgs boson hypothesis is performed. 


\begin{tabular}{|c|c|c|c|c|c|c|}
\hline \multirow[b]{3}{*}{$\mu[\mathrm{GeV}]$} & \multicolumn{6}{|c|}{ Neutral MSSM Higgs: low- $m_{\mathrm{H}}$ scenario } \\
\hline & \multicolumn{5}{|c|}{ Expected $\tan \beta$ limit } & \multirow{2}{*}{$\begin{array}{l}\text { Observed } \\
\tan \beta \text { limit }\end{array}$} \\
\hline & $-2 \sigma$ & $-1 \sigma$ & Median & $+1 \sigma$ & $+2 \sigma$ & \\
\hline 300 & $>3.4$ & $>4.7$ & $>6.0$ & $>7.3$ & $>8.6$ & $>6.6$ \\
\hline 400 & $>3.2$ & $>4.7$ & $>6.0$ & $>7.2$ & $>8.8$ & $>6.7$ \\
\hline 500 & $>3.3$ & $>4.9$ & $>6.1$ & $>7.3$ & $>9.0$ & $>6.7$ \\
\hline 600 & $>3.4$ & $>5.0$ & $>6.2$ & $>7.4$ & $>9.0$ & $>6.8$ \\
\hline 700 & $>3.4$ & $>5.0$ & $>6.1$ & $>7.3$ & $>8.8$ & $>6.7$ \\
\hline 800 & $>4.0$ & $>5.2$ & $>6.1$ & $>7.3$ & $>8.2$ & $>6.7$ \\
\hline 1100 & $>4.5$ & $>5.3$ & $>6.3$ & $>7.5$ & $>8.1$ & $>6.8$ \\
\hline 1300 & $>4.7$ & $>5.4$ & $>6.4$ & $>7.6$ & $>8.2$ & $>6.9$ \\
\hline 1400 & $>4.9$ & $>5.5$ & $>6.5$ & $>7.7$ & $>8.1$ & $>6.9$ \\
\hline 1500 & $>5.0$ & $>5.6$ & $>6.6$ & $>7.8$ & $>8.2$ & $>7.0$ \\
\hline 1600 & $>5.1$ & $>5.7$ & $>6.7$ & $>7.9$ & $>8.3$ & $>7.0$ \\
\hline 1700 & $>5.2$ & $>5.8$ & $>6.8$ & $>7.9$ & $>8.3$ & $>7.0$ \\
\hline 1800 & $>5.3$ & $>5.9$ & $>6.8$ & $>7.9$ & $>8.3$ & $>7.0$ \\
\hline 1900 & $>5.2$ & $>5.9$ & $>6.8$ & $>7.9$ & $>8.3$ & $>6.8$ \\
\hline 2000 & $>5.3$ & $>5.9$ & $>6.7$ & $>7.9$ & $>8.1$ & $>6.6$ \\
\hline 2100 & $>5.2$ & $>5.8$ & $>6.6$ & $>8.1$ & $>8.0$ & $>6.4$ \\
\hline 2200 & $>5.1$ & $>5.6$ & $>6.7$ & $>8.1$ & $>8.3$ & $>6.3$ \\
\hline 2300 & $>5.0$ & $>5.5$ & $>6.9$ & $>8.1$ & $>8.9$ & $>6.7$ \\
\hline 2400 & $>4.7$ & $>5.4$ & $>7.0$ & $>8.0$ & $>9.4$ & $>7.3$ \\
\hline 2500 & $>4.4$ & $>5.7$ & $>7.0$ & $>8.0$ & $>9.6$ & $>7.4$ \\
\hline 2600 & $>4.0$ & $>6.1$ & $>6.9$ & $>7.9$ & $>9.9$ & $>7.2$ \\
\hline 2700 & $>3.8$ & $>6.1$ & $>6.8$ & $>7.8$ & $>9.8$ & $>6.9$ \\
\hline 2800 & $>5.1$ & $>5.9$ & $>6.7$ & $>7.7$ & $>8.3$ & $>6.8$ \\
\hline 2900 & $>5.2$ & $>5.8$ & $>6.5$ & $>7.5$ & $>7.8$ & $>6.5$ \\
\hline 3000 & $>5.1$ & $>5.6$ & $>6.3$ & $>7.4$ & $>7.6$ & $>6.3$ \\
\hline 3100 & $>4.6$ & $>5.3$ & $>6.1$ & $>7.1$ & $>7.5$ & $>6.0$ \\
\hline
\end{tabular}

Table 12. Expected and observed 95\% CL limits for $\tan \beta$ as a function of $\mu$, for the MSSM search in the low- $m_{\mathrm{H}}$ benchmark scenario. For the statistical procedure a test of the compatibility of the data to a signal of the three neutral Higgs bosons $\mathrm{h}, \mathrm{H}$ and A compared to a SM Higgs boson hypothesis is performed. 


\begin{tabular}{|c|ccccc|c|}
\hline \multicolumn{7}{|c|}{$\sigma(\operatorname{gg} \phi) \cdot \mathcal{B}(\tau \tau): \sqrt{s}=8 \mathrm{TeV}$} \\
\cline { 2 - 7 }$m_{\phi}[\mathrm{GeV}]$ & $-2 \sigma$ & $-1 \sigma$ & Median & $+1 \sigma$ & $+2 \sigma$ & limit \\
\hline 90 & 16.75 & 20.36 & 25.45 & 31.65 & 37.34 & 50.21 \\
100 & 13.54 & 16.66 & 21.18 & 27.00 & 34.57 & 31.33 \\
120 & 2.96 & 3.88 & 5.14 & 6.65 & 8.09 & 7.38 \\
130 & 1.51 & 2.05 & 2.75 & 3.63 & 4.55 & 4.39 \\
140 & $9.42 \times 10^{-1}$ & 1.24 & 1.68 & 2.23 & 2.82 & 2.27 \\
160 & $4.74 \times 10^{-1}$ & $6.15 \times 10^{-1}$ & $8.55 \times 10^{-1}$ & 1.14 & 1.50 & $8.45 \times 10^{-1}$ \\
180 & $3.12 \times 10^{-1}$ & $4.05 \times 10^{-1}$ & $5.52 \times 10^{-1}$ & $7.41 \times 10^{-1}$ & $9.74 \times 10^{-1}$ & $5.49 \times 10^{-1}$ \\
200 & $2.37 \times 10^{-1}$ & $3.08 \times 10^{-1}$ & $4.19 \times 10^{-1}$ & $5.56 \times 10^{-1}$ & $7.48 \times 10^{-1}$ & $5.17 \times 10^{-1}$ \\
250 & $1.30 \times 10^{-1}$ & $1.69 \times 10^{-1}$ & $2.28 \times 10^{-1}$ & $3.11 \times 10^{-1}$ & $3.95 \times 10^{-1}$ & $3.15 \times 10^{-1}$ \\
300 & $7.95 \times 10^{-2}$ & $1.05 \times 10^{-1}$ & $1.43 \times 10^{-1}$ & $1.97 \times 10^{-1}$ & $2.53 \times 10^{-1}$ & $1.50 \times 10^{-1}$ \\
350 & $6.05 \times 10^{-2}$ & $7.71 \times 10^{-2}$ & $1.02 \times 10^{-1}$ & $1.38 \times 10^{-1}$ & $1.80 \times 10^{-1}$ & $1.12 \times 10^{-1}$ \\
400 & $4.68 \times 10^{-2}$ & $6.01 \times 10^{-2}$ & $7.94 \times 10^{-2}$ & $1.08 \times 10^{-1}$ & $1.41 \times 10^{-1}$ & $1.03 \times 10^{-1}$ \\
450 & $3.76 \times 10^{-2}$ & $4.77 \times 10^{-2}$ & $6.36 \times 10^{-2}$ & $8.39 \times 10^{-2}$ & $1.11 \times 10^{-1}$ & $6.07 \times 10^{-2}$ \\
500 & $3.20 \times 10^{-2}$ & $4.01 \times 10^{-2}$ & $5.31 \times 10^{-2}$ & $7.14 \times 10^{-2}$ & $9.28 \times 10^{-2}$ & $3.83 \times 10^{-2}$ \\
600 & $1.97 \times 10^{-2}$ & $2.54 \times 10^{-2}$ & $3.49 \times 10^{-2}$ & $4.70 \times 10^{-2}$ & $6.22 \times 10^{-2}$ & $1.93 \times 10^{-2}$ \\
700 & $1.49 \times 10^{-2}$ & $1.95 \times 10^{-2}$ & $2.72 \times 10^{-2}$ & $3.71 \times 10^{-2}$ & $4.73 \times 10^{-2}$ & $1.44 \times 10^{-2}$ \\
800 & $1.19 \times 10^{-2}$ & $1.53 \times 10^{-2}$ & $2.09 \times 10^{-2}$ & $2.89 \times 10^{-2}$ & $3.91 \times 10^{-2}$ & $1.12 \times 10^{-2}$ \\
900 & $8.55 \times 10^{-3}$ & $1.11 \times 10^{-2}$ & $1.51 \times 10^{-2}$ & $2.11 \times 10^{-2}$ & $2.89 \times 10^{-2}$ & $9.39 \times 10^{-3}$ \\
1000 & $7.47 \times 10^{-3}$ & $9.80 \times 10^{-3}$ & $1.30 \times 10^{-2}$ & $1.86 \times 10^{-2}$ & $2.54 \times 10^{-2}$ & $8.50 \times 10^{-3}$ \\
\hline
\end{tabular}

Table 13. Expected and observed 95\% CL upper limits for $\sigma(\operatorname{gg} \phi) \cdot \mathcal{B}(\tau \tau)(\mathrm{pb})$ at $8 \mathrm{TeV}$ centerof-mass energy as a function of the Higgs mass $m_{\phi}$, where $\phi$ denotes a generic Higgs-like state. The expected and observed limits are computed using the test statistics given by eq. (7.1). For the expected limits, the observed data have been replaced by a representative dataset which not only contains the contribution from background processes but also a SM Higgs boson with a mass of $125 \mathrm{GeV}$. 


\begin{tabular}{|c|ccccc|c|}
\hline \multicolumn{7}{c}{$\sigma(\mathrm{bb} \phi) \cdot \mathcal{B}(\tau \tau): \sqrt{s}=8 \mathrm{TeV}$} \\
\cline { 2 - 6 }$m_{\phi}[\mathrm{GeV}]$ & $-2 \sigma$ & $-1 \sigma$ & Median & $+1 \sigma$ & $+2 \sigma$ & limit \\
\hline & 3.25 & 4.19 & 5.69 & 7.54 & 9.74 & 6.04 \\
100 & 2.43 & 3.07 & 4.14 & 5.69 & 7.19 & 4.13 \\
120 & 1.18 & 1.53 & 2.08 & 2.82 & 3.72 & 1.76 \\
130 & $8.31 \times 10^{-1}$ & 1.08 & 1.47 & 1.97 & 2.60 & 1.26 \\
140 & $6.21 \times 10^{-1}$ & $8.24 \times 10^{-1}$ & 1.10 & 1.46 & 1.91 & 1.25 \\
160 & $3.80 \times 10^{-1}$ & $4.99 \times 10^{-1}$ & $6.68 \times 10^{-1}$ & $8.94 \times 10^{-1}$ & 1.18 & $8.14 \times 10^{-1}$ \\
180 & $2.64 \times 10^{-1}$ & $3.45 \times 10^{-1}$ & $4.59 \times 10^{-1}$ & $6.27 \times 10^{-1}$ & $8.40 \times 10^{-1}$ & $6.59 \times 10^{-1}$ \\
200 & $1.92 \times 10^{-1}$ & $2.50 \times 10^{-1}$ & $3.39 \times 10^{-1}$ & $4.60 \times 10^{-1}$ & $6.10 \times 10^{-1}$ & $5.53 \times 10^{-1}$ \\
250 & $1.09 \times 10^{-1}$ & $1.41 \times 10^{-1}$ & $1.90 \times 10^{-1}$ & $2.62 \times 10^{-1}$ & $3.42 \times 10^{-1}$ & $2.16 \times 10^{-1}$ \\
300 & $7.33 \times 10^{-2}$ & $9.15 \times 10^{-2}$ & $1.25 \times 10^{-1}$ & $1.70 \times 10^{-1}$ & $2.24 \times 10^{-1}$ & $9.75 \times 10^{-2}$ \\
350 & $5.30 \times 10^{-2}$ & $6.58 \times 10^{-2}$ & $8.95 \times 10^{-2}$ & $1.20 \times 10^{-1}$ & $1.60 \times 10^{-1}$ & $6.38 \times 10^{-2}$ \\
400 & $4.09 \times 10^{-2}$ & $5.30 \times 10^{-2}$ & $6.92 \times 10^{-2}$ & $9.59 \times 10^{-2}$ & $1.26 \times 10^{-1}$ & $6.13 \times 10^{-2}$ \\
450 & $3.15 \times 10^{-2}$ & $4.09 \times 10^{-2}$ & $5.51 \times 10^{-2}$ & $7.68 \times 10^{-2}$ & $9.81 \times 10^{-2}$ & $4.31 \times 10^{-2}$ \\
500 & $2.70 \times 10^{-2}$ & $3.41 \times 10^{-2}$ & $4.63 \times 10^{-2}$ & $6.33 \times 10^{-2}$ & $8.44 \times 10^{-2}$ & $3.20 \times 10^{-2}$ \\
600 & $1.77 \times 10^{-2}$ & $2.34 \times 10^{-2}$ & $3.16 \times 10^{-2}$ & $4.37 \times 10^{-2}$ & $5.66 \times 10^{-2}$ & $2.03 \times 10^{-2}$ \\
700 & $1.35 \times 10^{-2}$ & $1.78 \times 10^{-2}$ & $2.44 \times 10^{-2}$ & $3.38 \times 10^{-2}$ & $4.40 \times 10^{-2}$ & $1.73 \times 10^{-2}$ \\
800 & $1.16 \times 10^{-2}$ & $1.49 \times 10^{-2}$ & $2.01 \times 10^{-2}$ & $2.73 \times 10^{-2}$ & $3.77 \times 10^{-2}$ & $1.65 \times 10^{-2}$ \\
900 & $8.81 \times 10^{-3}$ & $1.15 \times 10^{-2}$ & $1.52 \times 10^{-2}$ & $2.12 \times 10^{-2}$ & $3.00 \times 10^{-2}$ & $1.48 \times 10^{-2}$ \\
1000 & $8.15 \times 10^{-3}$ & $1.03 \times 10^{-2}$ & $1.37 \times 10^{-2}$ & $1.92 \times 10^{-2}$ & $2.72 \times 10^{-2}$ & $1.35 \times 10^{-2}$ \\
\hline
\end{tabular}

Table 14. Expected and observed 95\% CL upper limits for $\sigma(\mathrm{bb} \phi) \cdot \mathcal{B}(\tau \tau)(\mathrm{pb})$ at $8 \mathrm{TeV}$ centerof-mass energy as a function of the Higgs mass $m_{\phi}$, where $\phi$ denotes a generic Higgs-like state. The expected and observed limits are computed using the test statistics given by eq. (7.1). For the expected limits, the observed data have been replaced by a representative dataset which not only contains the contribution from background processes but also a SM Higgs boson with a mass of $125 \mathrm{GeV}$.

Open Access. This article is distributed under the terms of the Creative Commons Attribution License (CC-BY 4.0), which permits any use, distribution and reproduction in any medium, provided the original author(s) and source are credited.

\section{References}

[1] S.L. Glashow, Partial Symmetries of Weak Interactions, Nucl. Phys. 22 (1961) 579.

[2] S. Weinberg, A Model of Leptons, Phys. Rev. Lett. 19 (1967) 1264 [InSPIRE].

[3] A. Salam, Weak and electromagnetic interactions, in Elementary particle physics: relativistic groups and analyticity, Proccedings of the Eighth Nobel Symposium, Lerum Sweden (1968), N. Svartholm eds., Almqvist \& Wiksell, Stockholm Sweden (1968), pg. 367.

[4] F. Englert and R. Brout, Broken Symmetry and the Mass of Gauge Vector Mesons, Phys. Rev. Lett. 13 (1964) 321 [INSPIRE]. 
[5] P.W. Higgs, Broken symmetries, massless particles and gauge fields, Phys. Lett. 12 (1964) 132 [INSPIRE].

[6] P.W. Higgs, Broken Symmetries and the Masses of Gauge Bosons, Phys. Rev. Lett. 13 (1964) 508 [INSPIRE].

[7] G.S. Guralnik, C.R. Hagen and T.W.B. Kibble, Global Conservation Laws and Massless Particles, Phys. Rev. Lett. 13 (1964) 585 [InSPIRE].

[8] P.W. Higgs, Spontaneous Symmetry Breakdown without Massless Bosons, Phys. Rev. 145 (1966) 1156 [INSPIRE].

[9] T.W.B. Kibble, Symmetry breaking in nonAbelian gauge theories, Phys. Rev. 155 (1967) 1554 [INSPIRE].

[10] Y. Golfand and E.P. Likhtman, Extension of the Algebra of Poincaré Group Generators and Violation of p Invariance, JETP Lett. 13 (1971) 323 [INSPIRE].

[11] J. Wess and B. Zumino, Supergauge Transformations in Four-Dimensions, Nucl. Phys. B 70 (1974) 39 [INSPIRE].

[12] P. Fayet, Supergauge Invariant Extension of the Higgs Mechanism and a Model for the electron and Its Neutrino, Nucl. Phys. B 90 (1975) 104 [INSPIRE].

[13] P. Fayet, Spontaneously Broken Supersymmetric Theories of Weak, Electromagnetic and Strong Interactions, Phys. Lett. B 69 (1977) 489 [INSPIRE].

[14] CMS collaboration, The CMS experiment at the CERN LHC, 2008 JINST 3 S08004 [INSPIRE].

[15] CMS collaboration, Search for Neutral MSSM Higgs Bosons Decaying to Tau Pairs in pp Collisions at $\sqrt{s}=7 \mathrm{TeV}$, Phys. Rev. Lett. 106 (2011) 231801 [arXiv:1104.1619] [INSPIRE].

[16] CMS collaboration, Search for a Higgs boson decaying into a b-quark pair and produced in association with b quarks in proton-proton collisions at 7 TeV, Phys. Lett. B 722 (2013) 207 [arXiv: 1302.2892] [INSPIRE].

[17] ATLAS collaboration, Search for neutral MSSM Higgs bosons decaying to $\tau^{+} \tau^{-}$pairs in proton-proton collisions at $\sqrt{s}=7 \mathrm{TeV}$ with the ATLAS detector, Phys. Lett. B 705 (2011) 174 [arXiv: 1107.5003$]$ [INSPIRE].

[18] CDF collaboration, Search for Higgs bosons predicted in two-Higgs-doublet models via decays to tau lepton pairs in 1.96-TeV pp collisions, Phys. Rev. Lett. 103 (2009) 201801 [arXiv: 0906.1014] [INSPIRE].

[19] D0 collaboration, Search for neutral Higgs bosons in the multi-b-jet topology in $5.2 \mathrm{fb}^{-1}$ of $p \bar{p}$ collisions at $\sqrt{s}=1.96 \mathrm{TeV}$, Phys. Lett. B 698 (2011) 97 [arXiv:1011.1931] [INSPIRE].

[20] D0 collaboration, Search for Higgs bosons decaying to $\tau \tau$ pairs in $p \bar{p}$ collisions at $\sqrt{s}=1.96 \mathrm{TeV}$, Phys. Lett. B 707 (2012) 323 [arXiv:1106.4555] [INSPIRE].

[21] CDF collaboration, Search for Higgs Bosons Produced in Association with b-quarks, Phys. Rev. D 85 (2012) 032005 [arXiv:1106.4782] [INSPIRE].

[22] ALEPH, DELPHI, L3, OPAL and LEP Working Group for Higgs Boson SEARCHES collaborations, S. Schael et al., Search for neutral MSSM Higgs bosons at LEP, Eur. Phys. J. C 47 (2006) 547 [hep-ex/0602042] [INSPIRE]. 
[23] G. Degrassi, S. Heinemeyer, W. Hollik, P. Slavich and G. Weiglein, Towards high precision predictions for the MSSM Higgs sector, Eur. Phys. J. C 28 (2003) 133 [hep-ph/0212020] [INSPIRE].

[24] M.S. Carena, S. Heinemeyer, C.E.M. Wagner and G. Weiglein, Suggestions for benchmark scenarios for MSSM Higgs boson searches at hadron colliders, Eur. Phys. J. C 26 (2003) 601 [hep-ph/0202167] [INSPIRE].

[25] M.S. Carena, S. Heinemeyer, C.E.M. Wagner and G. Weiglein, MSSM Higgs boson searches at the Tevatron and the LHC: Impact of different benchmark scenarios, Eur. Phys. J. C 45 (2006) 797 [hep-ph/0511023] [INSPIRE].

[26] ATLAS collaboration, Observation of a new particle in the search for the Standard Model Higgs boson with the ATLAS detector at the LHC, Phys. Lett. B 716 (2012) 1 [arXiv: 1207.7214] [INSPIRE].

[27] CMS collaboration, Observation of a new boson at a mass of $125 \mathrm{GeV}$ with the CMS experiment at the LHC, Phys. Lett. B 716 (2012) 30 [arXiv:1207.7235] [InSPIRE].

[28] CMS collaboration, Observation of a new boson with mass near $125 \mathrm{GeV}$ in pp collisions at $\sqrt{s}=7$ and $8 \mathrm{TeV}$, JHEP 06 (2013) 081 [arXiv:1303.4571] [INSPIRE].

[29] CMS collaboration, Evidence for the $125 \mathrm{GeV}$ Higgs boson decaying to a pair of $\tau$ leptons, JHEP 05 (2014) 104 [arXiv: 1401.5041] [INSPIRE].

[30] S. Heinemeyer, O. Stàl and G. Weiglein, Interpreting the LHC Higgs Search Results in the MSSM, Phys. Lett. B 710 (2012) 201 [arXiv:1112.3026] [inSPIRE].

[31] M. Carena et al., MSSM Higgs Boson Searches at the LHC: Benchmark Scenarios after the Discovery of a Higgs-like Particle, Eur. Phys. J. C 73 (2013) 2552 [arXiv:1302.7033] [INSPIRE].

[32] LHC Higgs Cross Section Working Group collaboration, Handbook of LHC Higgs Cross Sections: 1. Inclusive Observables, CERN-2011-002 (2011) [arXiv:1101.0593] [INSPIRE].

[33] M. Spira, A. Djouadi, D. Graudenz and P.M. Zerwas, Higgs boson production at the LHC, Nucl. Phys. B 453 (1995) 17 [hep-ph/9504378] [INSPIRE].

[34] M. Spira, HIGLU: A program for the calculation of the total Higgs production cross-section at hadron colliders via gluon fusion including QCD corrections, DESY Report DESY-T-95-05 (1995) [hep-ph/9510347].

[35] R.V. Harlander and W.B. Kilgore, Next-to-next-to-leading order Higgs production at hadron colliders, Phys. Rev. Lett. 88 (2002) 201801 [hep-ph/0201206] [INSPIRE].

[36] C. Anastasiou and K. Melnikov, Higgs boson production at hadron colliders in NNLO QCD, Nucl. Phys. B 646 (2002) 220 [hep-ph/0207004] [INSPIRE].

[37] V. Ravindran, J. Smith and W.L. van Neerven, NNLO corrections to the total cross-section for Higgs boson production in hadron hadron collisions, Nucl. Phys. B 665 (2003) 325 [hep-ph/0302135] [INSPIRE].

[38] R.V. Harlander and W.B. Kilgore, Production of a pseudoscalar Higgs boson at hadron colliders at next-to-next-to leading order, JHEP 10 (2002) 017 [hep-ph/0208096] [INSPIRE].

[39] C. Anastasiou and K. Melnikov, Pseudoscalar Higgs boson production at hadron colliders in NNLO QCD, Phys. Rev. D 67 (2003) 037501 [hep-ph/0208115] [INSPIRE]. 
[40] R.V. Harlander, S. Liebler and H. Mantler, SusHi: A program for the calculation of Higgs production in gluon fusion and bottom-quark annihilation in the Standard Model and the MSSM, Computer Physics Communications 184 (2013) 1605 [arXiv:1212.3249] [INSPIRE].

[41] R.V. Harlander and M. Steinhauser, Supersymmetric Higgs production in gluon fusion at next-to-leading order, JHEP 09 (2004) 066 [hep-ph/0409010] [INSPIRE].

[42] R. Harlander and P. Kant, Higgs production and decay: Analytic results at next-to-leading order QCD, JHEP 12 (2005) 015 [hep-ph/0509189] [INSPIRE].

[43] G. Degrassi and P. Slavich, NLO QCD bottom corrections to Higgs boson production in the MSSM, JHEP 11 (2010) 044 [arXiv:1007.3465] [INSPIRE].

[44] G. Degrassi, S. Di Vita and P. Slavich, NLO QCD corrections to pseudoscalar Higgs production in the MSSM, JHEP 08 (2011) 128 [arXiv:1107.0914] [INSPIRE].

[45] G. Degrassi, S. Di Vita and P. Slavich, On the NLO QCD Corrections to the Production of the Heaviest Neutral Higgs Scalar in the MSSM, Eur. Phys. J. C 72 (2012) 2032 [arXiv:1204.1016] [INSPIRE].

[46] U. Aglietti, R. Bonciani, G. Degrassi and A. Vicini, Two loop light fermion contribution to Higgs production and decays, Phys. Lett. B 595 (2004) 432 [hep-ph/0404071] [INSPIRE].

[47] R. Bonciani, G. Degrassi and A. Vicini, On the Generalized Harmonic Polylogarithms of One Complex Variable, Comput. Phys. Commun. 182 (2011) 1253 [arXiv:1007.1891] [InSPIRE].

[48] S. Dittmaier, M. Krämer and M. Spira, Higgs radiation off bottom quarks at the Tevatron and the CERN LHC, Phys. Rev. D 70 (2004) 074010 [hep-ph/0309204] [INSPIRE].

[49] S. Dawson, C.B. Jackson, L. Reina and D. Wackeroth, Exclusive Higgs boson production with bottom quarks at hadron colliders, Phys. Rev. D 69 (2004) 074027 [hep-ph/0311067] [INSPIRE].

[50] R.V. Harlander and W.B. Kilgore, Higgs boson production in bottom quark fusion at next-to-next-to leading order, Phys. Rev. D 68 (2003) 013001 [hep-ph/0304035] [INSPIRE].

[51] R.V. Harlander, M. Krämer and M. Schumacher, Bottom-quark associated Higgs-boson production: reconciling the four- and five-flavour scheme approach, CERN-PH-TH-2011-134, FR-PHENO-2011-009, TTK-11-17, WUB-11-04 (2011) [arXiv:1112 .3478].

[52] S. Heinemeyer, W. Hollik and G. Weiglein, FeynHiggs: A Program for the calculation of the masses of the neutral CP even Higgs bosons in the MSSM, Comput. Phys. Commun. 124 (2000) 76 [hep-ph/9812320] [INSPIRE].

[53] S. Heinemeyer, W. Hollik and G. Weiglein, The Masses of the neutral CP-even Higgs bosons in the MSSM: Accurate analysis at the two loop level, Eur. Phys. J. C 9 (1999) 343 [hep-ph/9812472] [INSPIRE].

[54] M. Frank et al., The Higgs Boson Masses and Mixings of the Complex MSSM in the Feynman-Diagrammatic Approach, JHEP 02 (2007) 047 [hep-ph/0611326] [INSPIRE].

[55] M. Spira, QCD effects in Higgs physics, Fortsch. Phys. 46 (1998) 203 [hep-ph/9705337] [INSPIRE].

[56] A. Djouadi, J. Kalinowski and M. Spira, HDECAY: A Program for Higgs boson decays in the standard model and its supersymmetric extension, Comput. Phys. Commun. 108 (1998) 56 [hep-ph/9704448] [INSPIRE]. 
[57] A. Djouadi, M.M. Mühlleitner and M. Spira, Decays of supersymmetric particles: The Program SUSY-HIT (SUspect-SdecaY-HDECAY-InTerface), Acta Phys. Polon. B 38 (2007) 635 [hep-ph/0609292] [INSPIRE].

[58] LHC Higgs Cross Section Working Group collaboration, Handbook of LHC Higgs Cross Sections: 3. Higgs Properties, CERN-2013-004 (2014) [arXiv:1307.1347] [INSPIRE].

[59] CMS collaboration, Particle-Flow Event Reconstruction in CMS and Performance for Jets, Taus and MET, CMS-PAS-PFT-09-001 (2009).

[60] CMS collaboration, Commissioning of the Particle-flow Event Reconstruction with the first LHC collisions recorded in the CMS detector, CMS-PAS-PFT-10-001 (2010).

[61] M. Cacciari, G.P. Salam and G. Soyez, FastJet User Manual, Eur. Phys. J. C 72 (2012) 1896 [arXiv: 1111.6097] [INSPIRE].

[62] M. Cacciari and G.P. Salam, Dispelling the $N^{3}$ myth for the $k_{t}$ jet-finder, Phys. Lett. B 641 (2006) 57 [hep-ph/0512210] [INSPIRE].

[63] M. Cacciari and G.P. Salam, Pileup subtraction using jet areas, Phys. Lett. B 659 (2008) 119 [arXiv:0707.1378] [INSPIRE].

[64] CMS collaboration, Determination of Jet Energy Calibration and Transverse Momentum Resolution in CMS, 2011 JINST 6 P11002 [arXiv:1107.4277] [INSPIRE].

[65] CMS collaboration, Identification of b-quark jets with the CMS experiment, 2013 JINST 8 P04013 [arXiv: 1211.4462] [INSPIRE].

[66] CMS collaboration, Performance of tau-lepton reconstruction and identification in CMS, 2012 JINST 7 P01001 [arXiv:1109.6034] [INSPIRE].

[67] CMS collaboration, MET performance in 8 TeV data, CMS-PAS-JME-12-002 (2012).

[68] T. Sjöstrand, S. Mrenna and P.Z. Skands, PYTHIA 6.4 Physics and Manual, JHEP 05 (2006) 026 [hep-ph/0603175] [INSPIRE].

[69] J. Alwall, M. Herquet, F. Maltoni, O. Mattelaer and T. Stelzer, MadGraph 5: Going Beyond, JHEP 06 (2011) 128 [arXiv:1106.0522] [INSPIRE].

[70] P. Nason, A New method for combining NLO QCD with shower Monte Carlo algorithms, JHEP 11 (2004) 040 [hep-ph/0409146] [INSPIRE].

[71] S. Frixione, P. Nason and C. Oleari, Matching NLO QCD computations with Parton Shower simulations: the POWHEG method, JHEP 11 (2007) 070 [arXiv: 0709.2092] [INSPIRE].

[72] S. Alioli, P. Nason, C. Oleari and E. Re, NLO single-top production matched with shower in POWHEG: s- and t-channel contributions, JHEP 09 (2009) 111 [Erratum ibid. 1002 (2010) 011] [arXiv:0907.4076] [INSPIRE].

[73] S. Alioli, P. Nason, C. Oleari and E. Re, A general framework for implementing NLO calculations in shower Monte Carlo programs: the POWHEG BOX, JHEP 06 (2010) 043 [arXiv: 1002.2581] [INSPIRE].

[74] N. Davidson, G. Nanava, T. Przedzinski, E. Richter-Was and Z. Was, Universal Interface of TAUOLA Technical and Physics Documentation, Comput. Phys. Commun. 183 (2012) 821 [arXiv: 1002.0543] [INSPIRE].

[75] GEANT4 collaboration, S. Agostinelli et al., GEANT4 - a simulation toolkit, Nucl. Instrum. Meth. A 506 (2003) 250 [INSPIRE]. 
[76] CMS collaboration, Measurements of Inclusive $W$ and $Z$ Cross Sections in pp Collisions at $\sqrt{s}=7 \mathrm{TeV}$, JHEP 01 (2011) 080 [arXiv:1012.2466] [INSPIRE].

[77] CMS collaboration, Electron reconstruction and identification at $\sqrt{s}=7 \mathrm{TeV}$, CMS-PAS-EGM-10-004 (2010).

[78] CMS collaboration, Performance of CMS muon reconstruction in pp collision events at $\sqrt{s}=7 \mathrm{TeV}, 2012$ JINST 7 P10002 [arXiv:1206.4071] [INSPIRE].

[79] CMS collaboration, Measurement of the Inclusive Z Cross Section via Decays to Tau Pairs in pp Collisions at $\sqrt{s}=7 \mathrm{TeV}$, JHEP 08 (2011) 117 [arXiv:1104.1617] [INSPIRE].

[80] A. Hocker et al., TMVA - Toolkit for Multivariate Data Analysis, PoS (ACAT) 040 [physics/0703039] [INSPIRE].

[81] CMS collaboration, Absolute Calibration of the Luminosity Measurement at CMS: Winter 2012 Update, CMS-PAS-SMP-12-008 (2012).

[82] CMS collaboration, CMS Luminosity Based on Pixel Cluster Counting - Summer 2013 Update, CMS-PAS-LUM-13-001 (2013).

[83] A.D. Martin, W.J. Stirling, R.S. Thorne and G. Watt, Parton distributions for the LHC, Eur. Phys. J. C 63 (2009) 189 [arXiv:0901.0002] [inSPIRE].

[84] A.D. Martin, W.J. Stirling, R.S. Thorne and G. Watt, Uncertainties on $\alpha_{s}$ in global PDF analyses and implications for predicted hadronic cross sections, Eur. Phys. J. C 64 (2009) 653 [arXiv: 0905.3531] [INSPIRE].

[85] ATLAS, CMS and LHC Higgs Combination Group collaborations, Procedure for the LHC Higgs boson search combination in Summer 2011, ATL-PHYS-PUB 2011-11, CMS NOTE 2011/005 (2011).

[86] R.J. Barlow and C. Beeston, Fitting using finite Monte Carlo samples, Comput. Phys. Commun. 77 (1993) 219 [INSPIRE].

[87] T. Junk, Confidence level computation for combining searches with small statistics, Nucl. Instrum. Meth. A 434 (1999) 435 [hep-ex/9902006] [INSPIRE].

[88] A.L. Read, Presentation of search results: The $C L_{s}$ technique, J. Phys. G 28 (2002) 2693 [INSPIRE]. 


\section{The CMS collaboration}

Yerevan Physics Institute, Yerevan, Armenia

V. Khachatryan, A.M. Sirunyan, A. Tumasyan

Institut für Hochenergiephysik der OeAW, Wien, Austria

W. Adam, T. Bergauer, M. Dragicevic, J. Erö, C. Fabjan ${ }^{1}$, M. Friedl, R. Frühwirth ${ }^{1}$, V.M. Ghete, C. Hartl, N. Hörmann, J. Hrubec, M. Jeitler ${ }^{1}$, W. Kiesenhofer, V. Knünz, M. Krammer ${ }^{1}$, I. Krätschmer, D. Liko, I. Mikulec, D. Rabady² , B. Rahbaran, H. Rohringer, R. Schöfbeck, J. Strauss, A. Taurok, W. Treberer-Treberspurg, W. Waltenberger, C.-E. Wulz ${ }^{1}$

National Centre for Particle and High Energy Physics, Minsk, Belarus

V. Mossolov, N. Shumeiko, J. Suarez Gonzalez

\section{Universiteit Antwerpen, Antwerpen, Belgium}

S. Alderweireldt, M. Bansal, S. Bansal, T. Cornelis, E.A. De Wolf, X. Janssen, A. Knutsson, S. Luyckx, S. Ochesanu, R. Rougny, M. Van De Klundert, H. Van Haevermaet, P. Van Mechelen, N. Van Remortel, A. Van Spilbeeck

\section{Vrije Universiteit Brussel, Brussel, Belgium}

F. Blekman, S. Blyweert, J. D'Hondt, N. Daci, N. Heracleous, J. Keaveney, S. Lowette, M. Maes, A. Olbrechts, Q. Python, D. Strom, S. Tavernier, W. Van Doninck, P. Van Mulders, G.P. Van Onsem, I. Villella

Université Libre de Bruxelles, Bruxelles, Belgium

C. Caillol, B. Clerbaux, G. De Lentdecker, D. Dobur, L. Favart, A.P.R. Gay, A. Grebenyuk, A. Léonard, A. Mohammadi, L. Perniè ${ }^{2}$, T. Reis, T. Seva, L. Thomas, C. Vander Velde, P. Vanlaer, J. Wang, F. Zenoni

\section{Ghent University, Ghent, Belgium}

V. Adler, K. Beernaert, L. Benucci, A. Cimmino, S. Costantini, S. Crucy, S. Dildick, A. Fagot, G. Garcia, J. Mccartin, A.A. Ocampo Rios, D. Ryckbosch, S. Salva Diblen, M. Sigamani, N. Strobbe, F. Thyssen, M. Tytgat, E. Yazgan, N. Zaganidis

Université Catholique de Louvain, Louvain-la-Neuve, Belgium

S. Basegmez, C. Beluffi ${ }^{3}$, G. Bruno, R. Castello, A. Caudron, L. Ceard, G.G. Da Silveira, C. Delaere, T. du Pree, D. Favart, L. Forthomme, A. Giammanco ${ }^{4}$, J. Hollar, A. Jafari, P. Jez, M. Komm, V. Lemaitre, C. Nuttens, D. Pagano, L. Perrini, A. Pin, K. Piotrzkowski, A. Popov ${ }^{5}$, L. Quertenmont, M. Selvaggi, M. Vidal Marono, J.M. Vizan Garcia

Université de Mons, Mons, Belgium

N. Beliy, T. Caebergs, E. Daubie, G.H. Hammad

Centro Brasileiro de Pesquisas Fisicas, Rio de Janeiro, Brazil

W.L. Aldá Júnior, G.A. Alves, L. Brito, M. Correa Martins Junior, T. Dos Reis Martins, C. Mora Herrera, M.E. Pol 
Universidade do Estado do Rio de Janeiro, Rio de Janeiro, Brazil

W. Carvalho, J. Chinellato ${ }^{6}$, A. Custódio, E.M. Da Costa, D. De Jesus Damiao, C. De Oliveira Martins, S. Fonseca De Souza, H. Malbouisson, D. Matos Figueiredo, L. Mundim, H. Nogima, W.L. Prado Da Silva, J. Santaolalla, A. Santoro, A. Sznajder, E.J. Tonelli Manganote ${ }^{6}$, A. Vilela Pereira

Universidade Estadual Paulista $^{a}$, Universidade Federal do ABC ${ }^{b}$, São Paulo, Brazil

C.A. Bernardes ${ }^{b}$, S. Dogra ${ }^{a}$, T.R. Fernandez Perez Tomei ${ }^{a}$, E.M. Gregores $^{b}$, P.G. Mercadante ${ }^{b}$, S.F. Novaes ${ }^{a}$, Sandra S. Padula ${ }^{a}$

Institute for Nuclear Research and Nuclear Energy, Sofia, Bulgaria

A. Aleksandrov, V. Genchev ${ }^{2}$, P. Iaydjiev, A. Marinov, S. Piperov, M. Rodozov, S. Stoykova, G. Sultanov, V. Tcholakov, M. Vutova

University of Sofia, Sofia, Bulgaria

A. Dimitrov, I. Glushkov, R. Hadjiiska, V. Kozhuharov, L. Litov, B. Pavlov, P. Petkov

Institute of High Energy Physics, Beijing, China

J.G. Bian, G.M. Chen, H.S. Chen, M. Chen, R. Du, C.H. Jiang, R. Plestina ${ }^{7}$, J. Tao, Z. Wang

State Key Laboratory of Nuclear Physics and Technology, Peking University, Beijing, China

C. Asawatangtrakuldee, Y. Ban, Q. Li, S. Liu, Y. Mao, S.J. Qian, D. Wang, W. Zou

Universidad de Los Andes, Bogota, Colombia

C. Avila, L.F. Chaparro Sierra, C. Florez, J.P. Gomez, B. Gomez Moreno, J.C. Sanabria

University of Split, Faculty of Electrical Engineering, Mechanical Engineering and Naval Architecture, Split, Croatia

N. Godinovic, D. Lelas, D. Polic, I. Puljak

University of Split, Faculty of Science, Split, Croatia

Z. Antunovic, M. Kovac

Institute Rudjer Boskovic, Zagreb, Croatia

V. Brigljevic, K. Kadija, J. Luetic, D. Mekterovic, L. Sudic

University of Cyprus, Nicosia, Cyprus

A. Attikis, G. Mavromanolakis, J. Mousa, C. Nicolaou, F. Ptochos, P.A. Razis

Charles University, Prague, Czech Republic

M. Bodlak, M. Finger, M. Finger Jr. ${ }^{8}$

Academy of Scientific Research and Technology of the Arab Republic of Egypt, Egyptian Network of High Energy Physics, Cairo, Egypt

Y. Assran ${ }^{9}$, A. Ellithi Kamel ${ }^{10}$, M.A. Mahmoud ${ }^{11}$, A. Radi ${ }^{12,13}$ 
National Institute of Chemical Physics and Biophysics, Tallinn, Estonia

M. Kadastik, M. Murumaa, M. Raidal, A. Tiko

Department of Physics, University of Helsinki, Helsinki, Finland

P. Eerola, G. Fedi, M. Voutilainen

Helsinki Institute of Physics, Helsinki, Finland

J. Härkönen, V. Karimäki, R. Kinnunen, M.J. Kortelainen, T. Lampén, K. Lassila-Perini,

S. Lehti, T. Lindén, P. Luukka, T. Mäenpää, T. Peltola, E. Tuominen, J. Tuominiemi,

E. Tuovinen, L. Wendland

Lappeenranta University of Technology, Lappeenranta, Finland

J. Talvitie, T. Tuuva

DSM/IRFU, CEA/Saclay, Gif-sur-Yvette, France

M. Besancon, F. Couderc, M. Dejardin, D. Denegri, B. Fabbro, J.L. Faure, C. Favaro,

F. Ferri, S. Ganjour, A. Givernaud, P. Gras, G. Hamel de Monchenault, P. Jarry, E. Locci,

J. Malcles, J. Rander, A. Rosowsky, M. Titov

Laboratoire Leprince-Ringuet, Ecole Polytechnique, IN2P3-CNRS, Palaiseau, France

S. Baffioni, F. Beaudette, P. Busson, C. Charlot, T. Dahms, M. Dalchenko, L. Dobrzynski, N. Filipovic, A. Florent, R. Granier de Cassagnac, L. Mastrolorenzo, P. Miné, C. Mironov, I.N. Naranjo, M. Nguyen, C. Ochando, P. Paganini, S. Regnard, R. Salerno, J.B. Sauvan, Y. Sirois, C. Veelken, Y. Yilmaz, A. Zabi

Institut Pluridisciplinaire Hubert Curien, Université de Strasbourg, Université de Haute Alsace Mulhouse, CNRS/IN2P3, Strasbourg, France

J.-L. Agram ${ }^{14}$, J. Andrea, A. Aubin, D. Bloch, J.-M. Brom, E.C. Chabert, C. Collard, E. Conte ${ }^{14}$, J.-C. Fontaine ${ }^{14}$, D. Gelé, U. Goerlach, C. Goetzmann, A.-C. Le Bihan, P. Van Hove

Centre de Calcul de l'Institut National de Physique Nucleaire et de Physique des Particules, CNRS/IN2P3, Villeurbanne, France

S. Gadrat

Université de Lyon, Université Claude Bernard Lyon 1, CNRS-IN2P3, Institut de Physique Nucléaire de Lyon, Villeurbanne, France

S. Beauceron, N. Beaupere, G. Boudoul'2, E. Bouvier, S. Brochet, C.A. Carrillo Montoya, J. Chasserat, R. Chierici, D. Contardo'2, P. Depasse, H. El Mamouni, J. Fan, J. Fay,

S. Gascon, M. Gouzevitch, B. Ille, T. Kurca, M. Lethuillier, L. Mirabito, S. Perries, J.D. Ruiz Alvarez, D. Sabes, L. Sgandurra, V. Sordini, M. Vander Donckt, P. Verdier, S. Viret, H. Xiao

Institute of High Energy Physics and Informatization, Tbilisi State University, Tbilisi, Georgia

Z. Tsamalaidze ${ }^{8}$ 


\section{RWTH Aachen University, I. Physikalisches Institut, Aachen, Germany}

C. Autermann, S. Beranek, M. Bontenackels, M. Edelhoff, L. Feld, O. Hindrichs, K. Klein, A. Ostapchuk, A. Perieanu, F. Raupach, J. Sammet, S. Schael, H. Weber, B. Wittmer, V. Zhukov ${ }^{5}$

\section{RWTH Aachen University, III. Physikalisches Institut A, Aachen, Germany}

M. Ata, M. Brodski, E. Dietz-Laursonn, D. Duchardt, M. Erdmann, R. Fischer, A. Güth, T. Hebbeker, C. Heidemann, K. Hoepfner, D. Klingebiel, S. Knutzen, P. Kreuzer, M. Merschmeyer, A. Meyer, P. Millet, M. Olschewski, K. Padeken, P. Papacz, H. Reithler, S.A. Schmitz, L. Sonnenschein, D. Teyssier, S. Thüer, M. Weber

\section{RWTH Aachen University, III. Physikalisches Institut B, Aachen, Germany}

V. Cherepanov, Y. Erdogan, G. Flügge, H. Geenen, M. Geisler, W. Haj Ahmad, A. Heister, F. Hoehle, B. Kargoll, T. Kress, Y. Kuessel, A. Künsken, J. Lingemann ${ }^{2}$, A. Nowack, I.M. Nugent, L. Perchalla, O. Pooth, A. Stahl

\section{Deutsches Elektronen-Synchrotron, Hamburg, Germany}

I. Asin, N. Bartosik, J. Behr, W. Behrenhoff, U. Behrens, A.J. Bell, M. Bergholz ${ }^{15}$, A. Bethani, K. Borras, A. Burgmeier, A. Cakir, L. Calligaris, A. Campbell, S. Choudhury, F. Costanza, C. Diez Pardos, S. Dooling, T. Dorland, G. Eckerlin, D. Eckstein, T. Eichhorn, G. Flucke, J. Garay Garcia, A. Geiser, P. Gunnellini, J. Hauk, G. Hellwig, M. Hempel ${ }^{15}$, D. Horton, H. Jung, A. Kalogeropoulos, M. Kasemann, P. Katsas, J. Kieseler, C. Kleinwort, D. Krücker, W. Lange, J. Leonard, K. Lipka, A. Lobanov, W. Lohmann ${ }^{15}$, B. Lutz, R. Mankel, I. Marfin ${ }^{15}$, I.-A. Melzer-Pellmann, A.B. Meyer, J. Mnich, A. Mussgiller, S. Naumann-Emme, A. Nayak, O. Novgorodova, E. Ntomari, H. Perrey, D. Pitzl, R. Placakyte, A. Raspereza, P.M. Ribeiro Cipriano, B. Roland, E. Ron, M.Ö. Sahin, J. Salfeld-Nebgen, P. Saxena, R. Schmidt ${ }^{15}$, T. Schoerner-Sadenius, M. Schröder, C. Seitz, S. Spannagel, A.D.R. Vargas Trevino, R. Walsh, C. Wissing

\section{University of Hamburg, Hamburg, Germany}

M. Aldaya Martin, V. Blobel, M. Centis Vignali, A.r. Draeger, J. Erfle, E. Garutti, K. Goebel, M. Görner, J. Haller, M. Hoffmann, R.S. Höing, H. Kirschenmann, R. Klanner, R. Kogler, J. Lange, T. Lapsien, T. Lenz, I. Marchesini, J. Ott, T. Peiffer, N. Pietsch, J. Poehlsen, T. Poehlsen, D. Rathjens, C. Sander, H. Schettler, P. Schleper, E. Schlieckau, A. Schmidt, M. Seidel, V. Sola, H. Stadie, G. Steinbrück, D. Troendle, E. Usai, L. Vanelderen, A. Vanhoefer

\section{Institut für Experimentelle Kernphysik, Karlsruhe, Germany}

C. Barth, C. Baus, J. Berger, C. Böser, E. Butz, T. Chwalek, W. De Boer, A. Descroix, A. Dierlamm, M. Feindt, F. Frensch, M. Giffels, F. Hartmann², T. Hauth², U. Husemann, I. Katkov ${ }^{5}$, A. Kornmayer ${ }^{2}$, E. Kuznetsova, P. Lobelle Pardo, M.U. Mozer, Th. Müller, A. Nürnberg, G. Quast, K. Rabbertz, F. Ratnikov, S. Röcker, H.J. Simonis, F.M. Stober, R. Ulrich, J. Wagner-Kuhr, S. Wayand, T. Weiler, R. Wolf 
Institute of Nuclear and Particle Physics (INPP), NCSR Demokritos, Aghia Paraskevi, Greece

G. Anagnostou, G. Daskalakis, T. Geralis, V.A. Giakoumopoulou, A. Kyriakis, D. Loukas, A. Markou, C. Markou, A. Psallidas, I. Topsis-Giotis

University of Athens, Athens, Greece

S. Kesisoglou, A. Panagiotou, N. Saoulidou, E. Stiliaris

University of Ioánnina, Ioánnina, Greece

X. Aslanoglou, I. Evangelou, G. Flouris, C. Foudas, P. Kokkas, N. Manthos, I. Papadopoulos, E. Paradas

Wigner Research Centre for Physics, Budapest, Hungary

G. Bencze, C. Hajdu, P. Hidas, D. Horvath ${ }^{16}$, F. Sikler, V. Veszpremi, G. Vesztergombi ${ }^{17}$, A.J. Zsigmond

Institute of Nuclear Research ATOMKI, Debrecen, Hungary

N. Beni, S. Czellar, J. Karancsi ${ }^{18}$, J. Molnar, J. Palinkas, Z. Szillasi

University of Debrecen, Debrecen, Hungary

P. Raics, Z.L. Trocsanyi, B. Ujvari

National Institute of Science Education and Research, Bhubaneswar, India S.K. Swain

Panjab University, Chandigarh, India

S.B. Beri, V. Bhatnagar, R. Gupta, U.Bhawandeep, A.K. Kalsi, M. Kaur, R. Kumar, M. Mittal, N. Nishu, J.B. Singh

University of Delhi, Delhi, India

Ashok Kumar, Arun Kumar, S. Ahuja, A. Bhardwaj, B.C. Choudhary, A. Kumar, S. Malhotra, M. Naimuddin, K. Ranjan, V. Sharma

Saha Institute of Nuclear Physics, Kolkata, India

S. Banerjee, S. Bhattacharya, K. Chatterjee, S. Dutta, B. Gomber, Sa. Jain, Sh. Jain, R. Khurana, A. Modak, S. Mukherjee, D. Roy, S. Sarkar, M. Sharan

Bhabha Atomic Research Centre, Mumbai, India

A. Abdulsalam, D. Dutta, S. Kailas, V. Kumar, A.K. Mohanty ${ }^{2}$, L.M. Pant, P. Shukla, A. Topkar

Tata Institute of Fundamental Research, Mumbai, India

T. Aziz, S. Banerjee, S. Bhowmik ${ }^{19}$, R.M. Chatterjee, R.K. Dewanjee, S. Dugad, S. Ganguly, S. Ghosh, M. Guchait, A. Gurtu ${ }^{20}$, G. Kole, S. Kumar, M. Maity ${ }^{19}$, G. Majumder, K. Mazumdar, G.B. Mohanty, B. Parida, K. Sudhakar, N. Wickramage ${ }^{21}$

Institute for Research in Fundamental Sciences (IPM), Tehran, Iran

H. Bakhshiansohi, H. Behnamian, S.M. Etesami22 ${ }^{22}$ A. Fahim ${ }^{23}$, R. Goldouzian, M. Khakzad, M. Mohammadi Najafabadi, M. Naseri, S. Paktinat Mehdiabadi, F. Rezaei Hosseinabadi, B. Safarzadeh ${ }^{24}$, M. Zeinali 
University College Dublin, Dublin, Ireland

M. Felcini, M. Grunewald

INFN Sezione di Bari ${ }^{a}$, Università di Bari ${ }^{b}$, Politecnico di Bari ${ }^{c}$, Bari, Italy

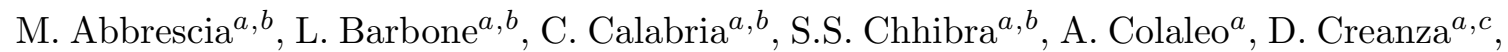
N. De Filippis ${ }^{a, c}$, M. De Palma ${ }^{a, b}$, L. Fiore ${ }^{a}$, G. Iaselli ${ }^{a, c}$, G. Maggi ${ }^{a, c}$, M. Maggi $^{a}, \mathrm{~S} \mathrm{My}^{a, c}$, S. Nuzzo ${ }^{a, b}$, A. Pompili ${ }^{a, b}$, G. Pugliese ${ }^{a, c}$, R. Radogna ${ }^{a, b, 2}$, G. Selvaggi ${ }^{a, b}$, L. Silvestris ${ }^{a, 2}$, G. Singh ${ }^{a, b}$, R. Venditti ${ }^{a, b}$, G. Zito ${ }^{a}$

\section{INFN Sezione di Bologna ${ }^{a}$, Università di Bologna ${ }^{b}$, Bologna, Italy}

G. Abbiendi $^{a}$, A.C. Benvenuti ${ }^{a}$, D. Bonacorsi ${ }^{a}, b$, S. Braibant-Giacomelli ${ }^{a}, b$, L. Brigliadori ${ }^{a, b}$, R. Campanini ${ }^{a, b}$, P. Capiluppi ${ }^{a}, b$, A. Castro ${ }^{a, b}$, F.R. Cavallo ${ }^{a}$, G. Codispoti ${ }^{a, b}$, M. Cuffiani ${ }^{a, b}$, G.M. Dallavalle ${ }^{a}$, F. Fabbri $^{a}$, A. Fanfani ${ }^{a, b}$, D. Fasanella $^{a, b}$, P. Giacomelli ${ }^{a}$, C. Grandi ${ }^{a}$, L. Guiducci ${ }^{a}, b$, S. Marcellini ${ }^{a}$, G. Masetti ${ }^{a}$, A. Montanari ${ }^{a}$,

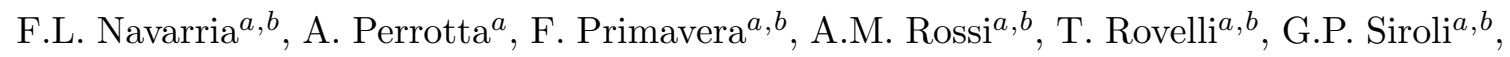
N. Tosi ${ }^{a, b}$, R. Travaglini ${ }^{a, b}$

INFN Sezione di Catania ${ }^{a}$, Università di Catania ${ }^{b}, \operatorname{CSFNSM}^{c}$, Catania, Italy S. Albergo ${ }^{a, b}$, G. Cappello ${ }^{a}$, M. Chiorboli ${ }^{a}, b$, S. Costa ${ }^{a, b}$, F. Giordano ${ }^{a, 2}$, R. Potenza ${ }^{a, b}$, A. Tricomi ${ }^{a, b}$, C. Tuve ${ }^{a, b}$

INFN Sezione di Firenze ${ }^{a}$, Università di Firenze ${ }^{b}$, Firenze, Italy

G. Barbagli ${ }^{a}$, V. Ciulli ${ }^{a, b}$, C. Civinini ${ }^{a}$, R. D'Alessandro ${ }^{a, b}$, E. Focardi ${ }^{a}, b$, E. Gallo ${ }^{a}$, S. Gonzi ${ }^{a, b}$, V. Gori ${ }^{a, b, 2}$, P. Lenzi ${ }^{a, b}$, M. Meschini ${ }^{a}$, S. Paoletti ${ }^{a}$, G. Sguazzoni ${ }^{a}$, A. Tropiano ${ }^{a, b}$

INFN Laboratori Nazionali di Frascati, Frascati, Italy

L. Benussi, S. Bianco, F. Fabbri, D. Piccolo

INFN Sezione di Genova ${ }^{a}$, Università di Genova ${ }^{b}$, Genova, Italy R. Ferretti ${ }^{a, b}$, F. Ferro $^{a}$, M. Lo Vetere ${ }^{a, b}$, E. Robutti ${ }^{a}$, S. Tosi ${ }^{a, b}$

INFN Sezione di Milano-Bicocca ${ }^{a}$, Università di Milano-Bicocca ${ }^{b}$, Milano, Italy

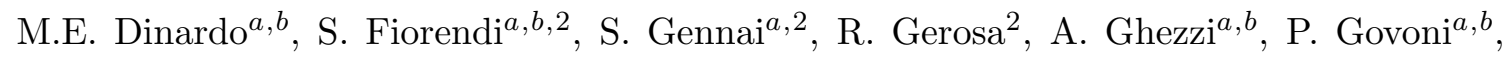
M.T. Lucchini ${ }^{a, b, 2}$, S. Malvezzi ${ }^{a}$, R.A. Manzoni ${ }^{a, b}$, A. Martelli ${ }^{a}, b$, B. Marzocchi, D. Menasce ${ }^{a}$, L. Moroni ${ }^{a}$, M. Paganoni ${ }^{a, b}$, D. Pedrini ${ }^{a}$, S. Ragazzi ${ }^{a, b}$, N. Redaelli ${ }^{a}$, T. Tabarelli de Fatis ${ }^{a, b}$

INFN Sezione di Napoli ${ }^{a}$, Università di Napoli 'Federico II ${ }^{b}$, Università della Basilicata (Potenza) ${ }^{c}$, Università G. Marconi (Roma) ${ }^{d}$, Napoli, Italy

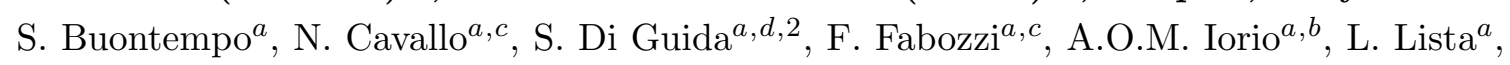
S. Meola ${ }^{a, d, 2}$, M. Merola ${ }^{a}$, P. Paolucci ${ }^{a, 2}$

INFN Sezione di Padova ${ }^{a}$, Università di Padova ${ }^{b}$, Università di Trento (Trento) ${ }^{c}$, Padova, Italy

P. Azzi ${ }^{a}$, N. Bacchetta ${ }^{a}$, D. Bisello ${ }^{a, b}$, A. Branca ${ }^{a, b}$, R. Carlin ${ }^{a, b}$, P. Chechia $^{a}$, M. Dall'Osso ${ }^{a, b}$, T. Dorigo ${ }^{a}$, U. Dosselli ${ }^{a}$, M. Galanti ${ }^{a, b}$, F. Gasparini ${ }^{a, b}$, U. Gasparini ${ }^{a, b}$, 
P. Giubilato ${ }^{a, b}$, A. Gozzelino ${ }^{a}$, K. Kanishchev ${ }^{a, c}$, S. Lacaprara ${ }^{a}$, M. Margoni ${ }^{a, b}$, A.T. Meneguzzo ${ }^{a, b}$, J. Pazzini ${ }^{a, b}$, N. Pozzobon ${ }^{a, b}$, P. Ronchese ${ }^{a, b}$, F. Simonetto ${ }^{a, b}$,

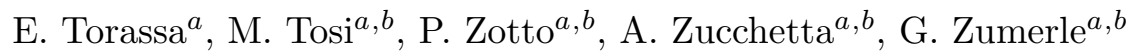

INFN Sezione di Pavia ${ }^{a}$, Università di Pavia ${ }^{b}$, Pavia, Italy

M. Gabusi ${ }^{a}, b$, S.P. Ratti ${ }^{a, b}$, V. Re ${ }^{a}$, C. Riccardi ${ }^{a, b}$, P. Salvini ${ }^{a}$, P. Vitulo ${ }^{a, b}$

INFN Sezione di Perugia ${ }^{a}$, Università di Perugia ${ }^{b}$, Perugia, Italy

M. Biasini ${ }^{a, b}$, G.M. Bilei ${ }^{a}$, D. Ciangottini ${ }^{a, b}$, L. Fanò ${ }^{a, b}$, P. Lariccia $^{a, b}$, G. Mantovani $^{a, b}{ }^{,}$

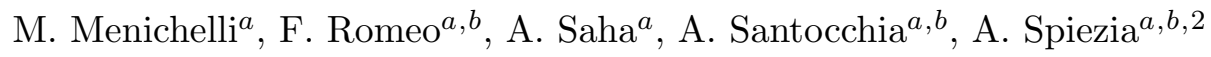

INFN Sezione di Pisa ${ }^{a}$, Università di Pisa ${ }^{b}$, Scuola Normale Superiore di Pisa ${ }^{c}$, Pisa, Italy

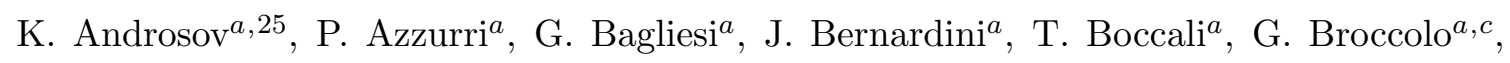

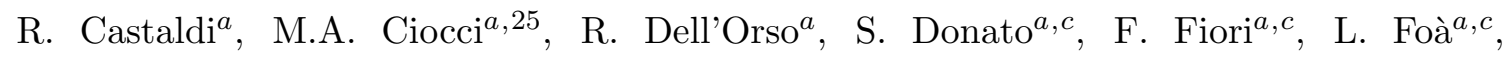

A. Giassi ${ }^{a}$, M.T. Grippo ${ }^{a, 25}$, F. Ligabue ${ }^{a, c}$, T. Lomtadze $^{a}$, L. Martini $^{a, b}$, A. Messineo $^{a, b}$, C.S. Moon ${ }^{a, 26}$, F. Palla ${ }^{a, 2}$, A. Rizzi ${ }^{a, b}$, A. Savoy-Navarro ${ }^{a, 27}$, A.T. Serban ${ }^{a}$, P. Spagnolo ${ }^{a}$,

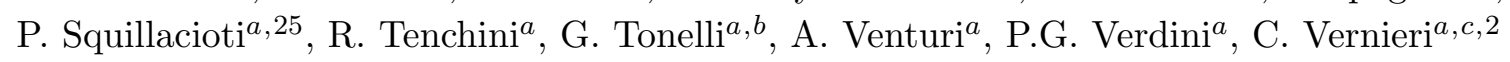

INFN Sezione di Roma ${ }^{a}$, Università di Roma ${ }^{b}$, Roma, Italy

L. Barone ${ }^{a, b}$, F. Cavallari ${ }^{a}$, G. D'imperio ${ }^{a, b}$, D. Del Re ${ }^{a, b}$, M. Diemoz ${ }^{a}$, M. Grassi ${ }^{a, b}$, C. Jorda ${ }^{a}$, E. Longo $^{a}, b$, F. Margaroli ${ }^{a, b}$, P. Meridiani ${ }^{a}$, F. Micheli ${ }^{a, b, 2}$, S. Nourbakhsh $^{a, b}$, G. Organtini ${ }^{a, b}$, R. Paramatti ${ }^{a}$, S. Rahatlou ${ }^{a, b}$, C. Rovelli ${ }^{a}$, F. Santanastasio ${ }^{a, b}$, L. Soffi ${ }^{a, b, 2}$, P. Traczyk ${ }^{a, b}$

INFN Sezione di Torino ${ }^{a}$, Università di Torino ${ }^{b}$, Università del Piemonte Orientale (Novara) ${ }^{c}$, Torino, Italy

N. Amapane ${ }^{a, b}$, R. Arcidiacono ${ }^{a, c}$, S. Argiro ${ }^{a, b, 2}$, M. Arneodo $^{a, c}$, R. Bellan $^{a, b}$, C. Biino $^{a}$,

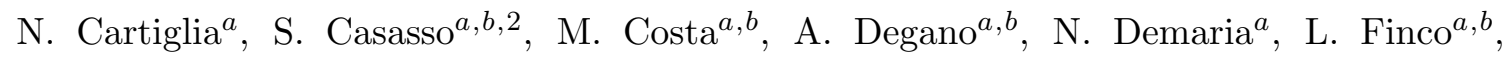
C. Mariotti ${ }^{a}$, S. Maselli ${ }^{a}$, E. Migliore ${ }^{a, b}$, V. Monaco ${ }^{a, b}$, M. Musich $^{a}$, M.M. Obertino $^{a, c, 2}$, G. Ortona ${ }^{a, b}$, L. Pacher ${ }^{a, b}$, N. Pastrone ${ }^{a}$, M. Pelliccioni ${ }^{a}$, G.L. Pinna Angioni ${ }^{a, b}$,

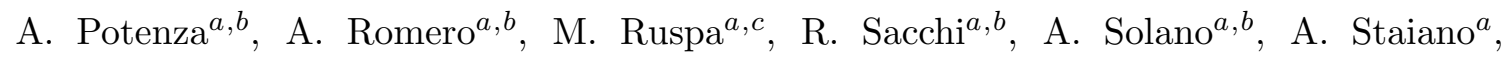
U. Tamponi ${ }^{a}$

INFN Sezione di Trieste ${ }^{a}$, Università di Trieste ${ }^{b}$, Trieste, Italy

S. Belforte ${ }^{a}$, V. Candelise ${ }^{a, b}$, M. Casarsa ${ }^{a}$, F. Cossutti ${ }^{a}$, G. Della Ricca ${ }^{a, b}$, B. Gobbo ${ }^{a}$,

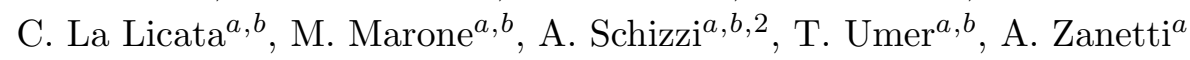

Kangwon National University, Chunchon, Korea

S. Chang, A. Kropivnitskaya, S.K. Nam

Kyungpook National University, Daegu, Korea

D.H. Kim, G.N. Kim, M.S. Kim, D.J. Kong, S. Lee, Y.D. Oh, H. Park, A. Sakharov, D.C. Son

Chonbuk National University, Jeonju, Korea

T.J. Kim 
Chonnam National University, Institute for Universe and Elementary Particles, Kwangju, Korea

J.Y. Kim, S. Song

Korea University, Seoul, Korea

S. Choi, D. Gyun, B. Hong, M. Jo, H. Kim, Y. Kim, B. Lee, K.S. Lee, S.K. Park, Y. Roh

University of Seoul, Seoul, Korea

M. Choi, J.H. Kim, I.C. Park, G. Ryu, M.S. Ryu

Sungkyunkwan University, Suwon, Korea

Y. Choi, Y.K. Choi, J. Goh, D. Kim, E. Kwon, J. Lee, H. Seo, I. Yu

Vilnius University, Vilnius, Lithuania

A. Juodagalvis

National Centre for Particle Physics, Universiti Malaya, Kuala Lumpur, Malaysia

J.R. Komaragiri, M.A.B. Md Ali

Centro de Investigacion y de Estudios Avanzados del IPN, Mexico City, Mexico

H. Castilla-Valdez, E. De La Cruz-Burelo, I. Heredia-de La Cruz ${ }^{28}$, A. Hernandez-Almada,

R. Lopez-Fernandez, A. Sanchez-Hernandez

Universidad Iberoamericana, Mexico City, Mexico

S. Carrillo Moreno, F. Vazquez Valencia

Benemerita Universidad Autonoma de Puebla, Puebla, Mexico

I. Pedraza, H.A. Salazar Ibarguen

Universidad Autónoma de San Luis Potosí, San Luis Potosí, Mexico

E. Casimiro Linares, A. Morelos Pineda

University of Auckland, Auckland, New Zealand

D. Krofcheck

University of Canterbury, Christchurch, New Zealand

P.H. Butler, S. Reucroft

National Centre for Physics, Quaid-I-Azam University, Islamabad, Pakistan

A. Ahmad, M. Ahmad, Q. Hassan, H.R. Hoorani, S. Khalid, W.A. Khan, T. Khurshid, M.A. Shah, M. Shoaib

National Centre for Nuclear Research, Swierk, Poland

H. Bialkowska, M. Bluj, B. Boimska, T. Frueboes, M. Górski, M. Kazana, K. Nawrocki,

K. Romanowska-Rybinska, M. Szleper, P. Zalewski

Institute of Experimental Physics, Faculty of Physics, University of Warsaw, Warsaw, Poland

G. Brona, K. Bunkowski, M. Cwiok, W. Dominik, K. Doroba, A. Kalinowski, M. Konecki,

J. Krolikowski, M. Misiura, M. Olszewski, W. Wolszczak 
Laboratório de Instrumentação e Física Experimental de Partículas, Lisboa, Portugal

P. Bargassa, C. Beirão Da Cruz E Silva, P. Faccioli, P.G. Ferreira Parracho, M. Gallinaro, L. Lloret Iglesias, F. Nguyen, J. Rodrigues Antunes, J. Seixas, J. Varela, P. Vischia

Joint Institute for Nuclear Research, Dubna, Russia

S. Afanasiev, P. Bunin, M. Gavrilenko, I. Golutvin, I. Gorbunov, A. Kamenev, V. Karjavin, V. Konoplyanikov, A. Lanev, A. Malakhov, V. Matveev ${ }^{29}$, P. Moisenz, V. Palichik, V. Perelygin, S. Shmatov, N. Skatchkov, V. Smirnov, A. Zarubin

Petersburg Nuclear Physics Institute, Gatchina (St. Petersburg), Russia

V. Golovtsov, Y. Ivanov, V. Kim ${ }^{30}$, P. Levchenko, V. Murzin, V. Oreshkin, I. Smirnov, V. Sulimov, L. Uvarov, S. Vavilov, A. Vorobyev, An. Vorobyev

Institute for Nuclear Research, Moscow, Russia

Yu. Andreev, A. Dermenev, S. Gninenko, N. Golubev, M. Kirsanov, N. Krasnikov, A. Pashenkov, D. Tlisov, A. Toropin

Institute for Theoretical and Experimental Physics, Moscow, Russia

V. Epshteyn, V. Gavrilov, N. Lychkovskaya, V. Popov, G. Safronov, S. Semenov, A. Spiridonov, V. Stolin, E. Vlasov, A. Zhokin

P.N. Lebedev Physical Institute, Moscow, Russia

V. Andreev, M. Azarkin, I. Dremin, M. Kirakosyan, A. Leonidov, G. Mesyats, S.V. Rusakov, A. Vinogradov

Skobeltsyn Institute of Nuclear Physics, Lomonosov Moscow State University, Moscow, Russia

A. Belyaev, E. Boos, V. Bunichev, M. Dubinin ${ }^{31}$, L. Dudko, A. Ershov, A. Gribushin, V. Klyukhin, O. Kodolova, I. Lokhtin, S. Obraztsov, S. Petrushanko, V. Savrin

State Research Center of Russian Federation, Institute for High Energy Physics, Protvino, Russia

I. Azhgirey, I. Bayshev, S. Bitioukov, V. Kachanov, A. Kalinin, D. Konstantinov, V. Krychkine, V. Petrov, R. Ryutin, A. Sobol, L. Tourtchanovitch, S. Troshin, N. Tyurin, A. Uzunian, A. Volkov

University of Belgrade, Faculty of Physics and Vinca Institute of Nuclear Sciences, Belgrade, Serbia

P. Adzic ${ }^{32}$, M. Ekmedzic, J. Milosevic, V. Rekovic

Centro de Investigaciones Energéticas Medioambientales y Tecnológicas (CIEMAT), Madrid, Spain

J. Alcaraz Maestre, C. Battilana, E. Calvo, M. Cerrada, M. Chamizo Llatas, N. Colino, B. De La Cruz, A. Delgado Peris, D. Domínguez Vázquez, A. Escalante Del Valle, C. Fernandez Bedoya, J.P. Fernández Ramos, J. Flix, M.C. Fouz, P. Garcia-Abia, O. Gonzalez Lopez, S. Goy Lopez, J.M. Hernandez, M.I. Josa, E. Navarro De Martino, A. Pérez-Calero Yzquierdo, J. Puerta Pelayo, A. Quintario Olmeda, I. Redondo, L. Romero, M.S. Soares 
Universidad Autónoma de Madrid, Madrid, Spain

C. Albajar, J.F. de Trocóniz, M. Missiroli, D. Moran

Universidad de Oviedo, Oviedo, Spain

H. Brun, J. Cuevas, J. Fernandez Menendez, S. Folgueras, I. Gonzalez Caballero

Instituto de Física de Cantabria (IFCA), CSIC-Universidad de Cantabria, Santander, Spain

J.A. Brochero Cifuentes, I.J. Cabrillo, A. Calderon, J. Duarte Campderros, M. Fernandez, G. Gomez, A. Graziano, A. Lopez Virto, J. Marco, R. Marco, C. Martinez Rivero, F. Matorras, F.J. Munoz Sanchez, J. Piedra Gomez, T. Rodrigo, A.Y. Rodríguez-Marrero, A. Ruiz-Jimeno, L. Scodellaro, I. Vila, R. Vilar Cortabitarte

\section{CERN, European Organization for Nuclear Research, Geneva, Switzerland}

D. Abbaneo, E. Auffray, G. Auzinger, M. Bachtis, P. Baillon, A.H. Ball, D. Barney, A. Benaglia, J. Bendavid, L. Benhabib, J.F. Benitez, C. Bernet ${ }^{7}$, G. Bianchi, P. Bloch, A. Bocci, A. Bonato, O. Bondu, C. Botta, H. Breuker, T. Camporesi, G. Cerminara, S. Colafranceschi ${ }^{33}$, M. D'Alfonso, D. d'Enterria, A. Dabrowski, A. David, F. De Guio, A. De Roeck, S. De Visscher, E. Di Marco, M. Dobson, M. Dordevic, N. Dupont-Sagorin, A. Elliott-Peisert, J. Eugster, G. Franzoni, W. Funk, D. Gigi, K. Gill, D. Giordano, M. Girone, F. Glege, R. Guida, S. Gundacker, M. Guthoff, J. Hammer, M. Hansen, P. Harris, J. Hegeman, V. Innocente, P. Janot, K. Kousouris, K. Krajczar, P. Lecoq, C. Lourenço, N. Magini, L. Malgeri, M. Mannelli, J. Marrouche, L. Masetti, F. Meijers, S. Mersi, E. Meschi, F. Moortgat, S. Morovic, M. Mulders, P. Musella, L. Orsini, L. Pape, E. Perez, L. Perrozzi, A. Petrilli, G. Petrucciani, A. Pfeiffer, M. Pierini, M. Pimiä, D. Piparo, M. Plagge, A. Racz, G. Rolandi ${ }^{34}$, M. Rovere, H. Sakulin, C. Schäfer, C. Schwick, A. Sharma, P. Siegrist, P. Silva, M. Simon, P. Sphicas ${ }^{35}$, D. Spiga, J. Steggemann, B. Stieger, M. Stoye, Y. Takahashi, D. Treille, A. Tsirou, G.I. Veres ${ }^{17}$, J.R. Vlimant, N. Wardle, H.K. Wöhri, H. Wollny, W.D. Zeuner

Paul Scherrer Institut, Villigen, Switzerland

W. Bertl, K. Deiters, W. Erdmann, R. Horisberger, Q. Ingram, H.C. Kaestli, D. Kotlinski, U. Langenegger, D. Renker, T. Rohe

Institute for Particle Physics, ETH Zurich, Zurich, Switzerland

F. Bachmair, L. Bäni, L. Bianchini, M.A. Buchmann, B. Casal, N. Chanon, G. Dissertori, M. Dittmar, M. Donegà, M. Dünser, P. Eller, C. Grab, D. Hits, J. Hoss, W. Lustermann, B. Mangano, A.C. Marini, P. Martinez Ruiz del Arbol, M. Masciovecchio, D. Meister, N. Mohr, C. Nägeli ${ }^{36}$, F. Nessi-Tedaldi, F. Pandolfi, F. Pauss, M. Peruzzi, M. Quittnat, L. Rebane, M. Rossini, A. Starodumov ${ }^{37}$, M. Takahashi, K. Theofilatos, R. Wallny, H.A. Weber

\section{Universität Zürich, Zurich, Switzerland}

C. Amsler ${ }^{38}$, M.F. Canelli, V. Chiochia, A. De Cosa, A. Hinzmann, T. Hreus, B. Kilminster, C. Lange, B. Millan Mejias, J. Ngadiuba, P. Robmann, F.J. Ronga, S. Taroni, M. Verzetti, Y. Yang 
National Central University, Chung-Li, Taiwan

M. Cardaci, K.H. Chen, C. Ferro, C.M. Kuo, W. Lin, Y.J. Lu, R. Volpe, S.S. Yu

National Taiwan University (NTU), Taipei, Taiwan

P. Chang, Y.H. Chang, Y.W. Chang, Y. Chao, K.F. Chen, P.H. Chen, C. Dietz, U. Grundler, W.-S. Hou, K.Y. Kao, Y.J. Lei, Y.F. Liu, R.-S. Lu, D. Majumder, E. Petrakou, Y.M. Tzeng, R. Wilken

Chulalongkorn University, Faculty of Science, Department of Physics, Bangkok, Thailand

B. Asavapibhop, N. Srimanobhas, N. Suwonjandee

Cukurova University, Adana, Turkey

A. Adiguzel, M.N. Bakirci ${ }^{39}$, S. Cerci ${ }^{40}$, C. Dozen, I. Dumanoglu, E. Eskut, S. Girgis, G. Gokbulut, E. Gurpinar, I. Hos, E.E. Kangal, A. Kayis Topaksu, G. Onengut ${ }^{41}$, K. Ozdemir, S. Ozturk ${ }^{39}$, A. Polatoz, D. Sunar Cerci ${ }^{40}$, B. Tali ${ }^{40}$, H. Topakli ${ }^{39}$, M. Vergili

Middle East Technical University, Physics Department, Ankara, Turkey

I.V. Akin, B. Bilin, S. Bilmis, H. Gamsizkan ${ }^{42}$, G. Karapinar ${ }^{43}$, K. Ocalan ${ }^{44}$, S. Sekmen, U.E. Surat, M. Yalvac, M. Zeyrek

Bogazici University, Istanbul, Turkey

E. Gülmez, B. Isildak ${ }^{45}$, M. Kaya ${ }^{46}$, O. Kaya ${ }^{47}$

Istanbul Technical University, Istanbul, Turkey

K. Cankocak, F.I. Vardarlı

National Scientific Center, Kharkov Institute of Physics and Technology, Kharkov, Ukraine

L. Levchuk, P. Sorokin

University of Bristol, Bristol, United Kingdom

J.J. Brooke, E. Clement, D. Cussans, H. Flacher, R. Frazier, J. Goldstein, M. Grimes, G.P. Heath, H.F. Heath, J. Jacob, L. Kreczko, C. Lucas, Z. Meng, D.M. Newbold ${ }^{48}$, S. Paramesvaran, A. Poll, S. Senkin, V.J. Smith, T. Williams

Rutherford Appleton Laboratory, Didcot, United Kingdom

K.W. Bell, A. Belyaev ${ }^{49}$, C. Brew, R.M. Brown, D.J.A. Cockerill, J.A. Coughlan, K. Harder, S. Harper, E. Olaiya, D. Petyt, C.H. Shepherd-Themistocleous, A. Thea, I.R. Tomalin, W.J. Womersley, S.D. Worm

\section{Imperial College, London, United Kingdom}

M. Baber, R. Bainbridge, O. Buchmuller, D. Burton, D. Colling, N. Cripps, M. Cutajar, P. Dauncey, G. Davies, M. Della Negra, P. Dunne, W. Ferguson, J. Fulcher, D. Futyan, A. Gilbert, G. Hall, G. Iles, M. Jarvis, G. Karapostoli, M. Kenzie, R. Lane, R. Lucas ${ }^{48}$, L. Lyons, A.-M. Magnan, S. Malik, B. Mathias, J. Nash, A. Nikitenko ${ }^{37}$, J. Pela, M. Pesaresi, K. Petridis, D.M. Raymond, S. Rogerson, A. Rose, C. Seez, P. Sharp ${ }^{\dagger}$, A. Tapper, M. Vazquez Acosta, T. Virdee, S.C. Zenz 
Brunel University, Uxbridge, United Kingdom

J.E. Cole, P.R. Hobson, A. Khan, P. Kyberd, D. Leggat, D. Leslie, W. Martin, I.D. Reid, P. Symonds, L. Teodorescu, M. Turner

\section{Baylor University, Waco, USA}

J. Dittmann, K. Hatakeyama, A. Kasmi, H. Liu, T. Scarborough

The University of Alabama, Tuscaloosa, USA

O. Charaf, S.I. Cooper, C. Henderson, P. Rumerio

\section{Boston University, Boston, USA}

A. Avetisyan, T. Bose, C. Fantasia, P. Lawson, C. Richardson, J. Rohlf, J. St. John, L. Sulak

\section{Brown University, Providence, USA}

J. Alimena, E. Berry, S. Bhattacharya, G. Christopher, D. Cutts, Z. Demiragli, N. Dhingra, A. Ferapontov, A. Garabedian, U. Heintz, G. Kukartsev, E. Laird, G. Landsberg, M. Luk, M. Narain, M. Segala, T. Sinthuprasith, T. Speer, J. Swanson

University of California, Davis, Davis, USA

R. Breedon, G. Breto, M. Calderon De La Barca Sanchez, S. Chauhan, M. Chertok, J. Conway, R. Conway, P.T. Cox, R. Erbacher, M. Gardner, W. Ko, R. Lander, T. Miceli, M. Mulhearn, D. Pellett, J. Pilot, F. Ricci-Tam, M. Searle, S. Shalhout, J. Smith, M. Squires, D. Stolp, M. Tripathi, S. Wilbur, R. Yohay

University of California, Los Angeles, USA

R. Cousins, P. Everaerts, C. Farrell, J. Hauser, M. Ignatenko, G. Rakness, E. Takasugi, V. Valuev, M. Weber

\section{University of California, Riverside, Riverside, USA}

K. Burt, R. Clare, J. Ellison, J.W. Gary, G. Hanson, J. Heilman, M. Ivova Rikova, P. Jandir, E. Kennedy, F. Lacroix, O.R. Long, A. Luthra, M. Malberti, H. Nguyen, M. Olmedo Negrete, A. Shrinivas, S. Sumowidagdo, S. Wimpenny

\section{University of California, San Diego, La Jolla, USA}

W. Andrews, J.G. Branson, G.B. Cerati, S. Cittolin, R.T. D'Agnolo, D. Evans, A. Holzner, R. Kelley, D. Klein, M. Lebourgeois, J. Letts, I. Macneill, D. Olivito, S. Padhi, C. Palmer, M. Pieri, M. Sani, V. Sharma, S. Simon, E. Sudano, M. Tadel, Y. Tu, A. Vartak, C. Welke, F. Würthwein, A. Yagil

\section{University of California, Santa Barbara, Santa Barbara, USA}

D. Barge, J. Bradmiller-Feld, C. Campagnari, T. Danielson, A. Dishaw, K. Flowers, M. Franco Sevilla, P. Geffert, C. George, F. Golf, L. Gouskos, J. Incandela, C. Justus, N. Mccoll, J. Richman, D. Stuart, W. To, C. West, J. Yoo

\section{California Institute of Technology, Pasadena, USA}

A. Apresyan, A. Bornheim, J. Bunn, Y. Chen, J. Duarte, A. Mott, H.B. Newman, C. Pena, C. Rogan, M. Spiropulu, V. Timciuc, R. Wilkinson, S. Xie, R.Y. Zhu 


\section{Carnegie Mellon University, Pittsburgh, USA}

V. Azzolini, A. Calamba, B. Carlson, T. Ferguson, Y. Iiyama, M. Paulini, J. Russ, H. Vogel, I. Vorobiev

University of Colorado at Boulder, Boulder, USA

J.P. Cumalat, W.T. Ford, A. Gaz, E. Luiggi Lopez, U. Nauenberg, J.G. Smith, K. Stenson, K.A. Ulmer, S.R. Wagner

Cornell University, Ithaca, USA

J. Alexander, A. Chatterjee, J. Chu, S. Dittmer, N. Eggert, N. Mirman, G. Nicolas Kaufman, J.R. Patterson, A. Ryd, E. Salvati, L. Skinnari, W. Sun, W.D. Teo, J. Thom, J. Thompson, J. Tucker, Y. Weng, L. Winstrom, P. Wittich

Fairfield University, Fairfield, USA

D. Winn

\section{Fermi National Accelerator Laboratory, Batavia, USA}

S. Abdullin, M. Albrow, J. Anderson, G. Apollinari, L.A.T. Bauerdick, A. Beretvas, J. Berryhill, P.C. Bhat, G. Bolla, K. Burkett, J.N. Butler, H.W.K. Cheung, F. Chlebana, S. Cihangir, V.D. Elvira, I. Fisk, J. Freeman, Y. Gao, E. Gottschalk, L. Gray, D. Green, S. Grünendahl, O. Gutsche, J. Hanlon, D. Hare, R.M. Harris, J. Hirschauer, B. Hooberman, S. Jindariani, M. Johnson, U. Joshi, K. Kaadze, B. Klima, B. Kreis, S. Kwan, J. Linacre, D. Lincoln, R. Lipton, T. Liu, J. Lykken, K. Maeshima, J.M. Marraffino, V.I. Martinez Outschoorn, S. Maruyama, D. Mason, P. McBride, P. Merkel, K. Mishra, S. Mrenna, Y. Musienko ${ }^{29}$, S. Nahn, C. Newman-Holmes, V. O'Dell, O. Prokofyev, E. Sexton-Kennedy, S. Sharma, A. Soha, W.J. Spalding, L. Spiegel, L. Taylor, S. Tkaczyk, N.V. Tran, L. Uplegger, E.W. Vaandering, R. Vidal, A. Whitbeck, J. Whitmore, F. Yang

\section{University of Florida, Gainesville, USA}

D. Acosta, P. Avery, P. Bortignon, D. Bourilkov, M. Carver, T. Cheng, D. Curry, S. Das, M. De Gruttola, G.P. Di Giovanni, R.D. Field, M. Fisher, I.K. Furic, J. Hugon, J. Konigsberg, A. Korytov, T. Kypreos, J.F. Low, K. Matchev, P. Milenovic ${ }^{50}$, G. Mitselmakher, L. Muniz, A. Rinkevicius, L. Shchutska, M. Snowball, D. Sperka, J. Yelton, M. Zakaria

Florida International University, Miami, USA

S. Hewamanage, S. Linn, P. Markowitz, G. Martinez, J.L. Rodriguez

Florida State University, Tallahassee, USA

T. Adams, A. Askew, J. Bochenek, B. Diamond, J. Haas, S. Hagopian, V. Hagopian, K.F. Johnson, H. Prosper, V. Veeraraghavan, M. Weinberg

Florida Institute of Technology, Melbourne, USA

M.M. Baarmand, M. Hohlmann, H. Kalakhety, F. Yumiceva

University of Illinois at Chicago (UIC), Chicago, USA

M.R. Adams, L. Apanasevich, V.E. Bazterra, D. Berry, R.R. Betts, I. Bucinskaite, R. Cavanaugh, O. Evdokimov, L. Gauthier, C.E. Gerber, D.J. Hofman, S. Khalatyan, P. Kurt, D.H. Moon, C. O'Brien, C. Silkworth, P. Turner, N. Varelas 
The University of Iowa, Iowa City, USA

E.A. Albayrak ${ }^{51}$, B. Bilki ${ }^{52}$, W. Clarida, K. Dilsiz, F. Duru, M. Haytmyradov, J.-P. Merlo, H. Mermerkaya ${ }^{53}$, A. Mestvirishvili, A. Moeller, J. Nachtman, H. Ogul, Y. Onel, F. Ozok ${ }^{51}$, A. Penzo, R. Rahmat, S. Sen, P. Tan, E. Tiras, J. Wetzel, T. Yetkin ${ }^{54}$, K. Yi

Johns Hopkins University, Baltimore, USA

B.A. Barnett, B. Blumenfeld, S. Bolognesi, D. Fehling, A.V. Gritsan, P. Maksimovic, C. Martin, M. Swartz

The University of Kansas, Lawrence, USA

P. Baringer, A. Bean, G. Benelli, C. Bruner, R.P. Kenny III, M. Malek, M. Murray,

D. Noonan, S. Sanders, J. Sekaric, R. Stringer, Q. Wang, J.S. Wood

Kansas State University, Manhattan, USA

A.F. Barfuss, I. Chakaberia, A. Ivanov, S. Khalil, M. Makouski, Y. Maravin, L.K. Saini, S. Shrestha, N. Skhirtladze, I. Svintradze

Lawrence Livermore National Laboratory, Livermore, USA

J. Gronberg, D. Lange, F. Rebassoo, D. Wright

University of Maryland, College Park, USA

A. Baden, A. Belloni, B. Calvert, S.C. Eno, J.A. Gomez, N.J. Hadley, R.G. Kellogg,

T. Kolberg, Y. Lu, M. Marionneau, A.C. Mignerey, K. Pedro, A. Skuja, M.B. Tonjes, S.C. Tonwar

Massachusetts Institute of Technology, Cambridge, USA

A. Apyan, R. Barbieri, G. Bauer, W. Busza, I.A. Cali, M. Chan, L. Di Matteo, V. Dutta, G. Gomez Ceballos, M. Goncharov, D. Gulhan, M. Klute, Y.S. Lai, Y.-J. Lee, A. Levin, P.D. Luckey, T. Ma, C. Paus, D. Ralph, C. Roland, G. Roland, G.S.F. Stephans, F. Stöckli, K. Sumorok, D. Velicanu, J. Veverka, B. Wyslouch, M. Yang, M. Zanetti, V. Zhukova

University of Minnesota, Minneapolis, USA

B. Dahmes, A. Gude, S.C. Kao, K. Klapoetke, Y. Kubota, J. Mans, N. Pastika, R. Rusack,

A. Singovsky, N. Tambe, J. Turkewitz

University of Mississippi, Oxford, USA

J.G. Acosta, S. Oliveros

University of Nebraska-Lincoln, Lincoln, USA

E. Avdeeva, K. Bloom, S. Bose, D.R. Claes, A. Dominguez, R. Gonzalez Suarez, J. Keller, D. Knowlton, I. Kravchenko, J. Lazo-Flores, S. Malik, F. Meier, G.R. Snow, M. Zvada

State University of New York at Buffalo, Buffalo, USA

J. Dolen, A. Godshalk, I. Iashvili, A. Kharchilava, A. Kumar, S. Rappoccio

Northeastern University, Boston, USA

G. Alverson, E. Barberis, D. Baumgartel, M. Chasco, J. Haley, A. Massironi, D.M. Morse,

D. Nash, T. Orimoto, D. Trocino, R.-J. Wang, D. Wood, J. Zhang 


\section{Northwestern University, Evanston, USA}

K.A. Hahn, A. Kubik, N. Mucia, N. Odell, B. Pollack, A. Pozdnyakov, M. Schmitt, S. Stoynev, K. Sung, M. Velasco, S. Won

\section{University of Notre Dame, Notre Dame, USA}

A. Brinkerhoff, K.M. Chan, A. Drozdetskiy, M. Hildreth, C. Jessop, D.J. Karmgard, N. Kellams, K. Lannon, W. Luo, S. Lynch, N. Marinelli, T. Pearson, M. Planer, R. Ruchti, N. Valls, M. Wayne, M. Wolf, A. Woodard

\section{The Ohio State University, Columbus, USA}

L. Antonelli, J. Brinson, B. Bylsma, L.S. Durkin, S. Flowers, C. Hill, R. Hughes, K. Kotov, T.Y. Ling, D. Puigh, M. Rodenburg, G. Smith, B.L. Winer, H. Wolfe, H.W. Wulsin

\section{Princeton University, Princeton, USA}

O. Driga, P. Elmer, P. Hebda, A. Hunt, S.A. Koay, P. Lujan, D. Marlow, T. Medvedeva, M. Mooney, J. Olsen, P. Piroué, X. Quan, H. Saka, D. Stickland ${ }^{2}$, C. Tully, J.S. Werner, A. Zuranski

\section{University of Puerto Rico, Mayaguez, USA}

E. Brownson, H. Mendez, J.E. Ramirez Vargas

\section{Purdue University, West Lafayette, USA}

V.E. Barnes, D. Benedetti, D. Bortoletto, M. De Mattia, L. Gutay, Z. Hu, M.K. Jha, M. Jones, K. Jung, M. Kress, N. Leonardo, D. Lopes Pegna, V. Maroussov, D.H. Miller, N. Neumeister, B.C. Radburn-Smith, X. Shi, I. Shipsey, D. Silvers, A. Svyatkovskiy, F. Wang, W. Xie, L. Xu, H.D. Yoo, J. Zablocki, Y. Zheng

\section{Purdue University Calumet, Hammond, USA}

N. Parashar, J. Stupak

\section{Rice University, Houston, USA}

A. Adair, B. Akgun, K.M. Ecklund, F.J.M. Geurts, W. Li, B. Michlin, B.P. Padley, R. Redjimi, J. Roberts, J. Zabel

\section{University of Rochester, Rochester, USA}

B. Betchart, A. Bodek, R. Covarelli, P. de Barbaro, R. Demina, Y. Eshaq, T. Ferbel, A. Garcia-Bellido, P. Goldenzweig, J. Han, A. Harel, A. Khukhunaishvili, G. Petrillo, D. Vishnevskiy

The Rockefeller University, New York, USA

R. Ciesielski, L. Demortier, K. Goulianos, G. Lungu, C. Mesropian

\section{Rutgers, The State University of New Jersey, Piscataway, USA}

S. Arora, A. Barker, J.P. Chou, C. Contreras-Campana, E. Contreras-Campana, D. Duggan, D. Ferencek, Y. Gershtein, R. Gray, E. Halkiadakis, D. Hidas, S. Kaplan, A. Lath, S. Panwalkar, M. Park, R. Patel, S. Salur, S. Schnetzer, S. Somalwar, R. Stone, S. Thomas, P. Thomassen, M. Walker 


\section{University of Tennessee, Knoxville, USA}

K. Rose, S. Spanier, A. York

\section{Texas A\&M University, College Station, USA}

O. Bouhali ${ }^{55}$, A. Castaneda Hernandez, R. Eusebi, W. Flanagan, J. Gilmore, T. Kamon ${ }^{56}$, V. Khotilovich, V. Krutelyov, R. Montalvo, I. Osipenkov, Y. Pakhotin, A. Perloff, J. Roe, A. Rose, A. Safonov, T. Sakuma, I. Suarez, A. Tatarinov

\section{Texas Tech University, Lubbock, USA}

N. Akchurin, C. Cowden, J. Damgov, C. Dragoiu, P.R. Dudero, J. Faulkner, K. Kovitanggoon, S. Kunori, S.W. Lee, T. Libeiro, I. Volobouev

\section{Vanderbilt University, Nashville, USA}

E. Appelt, A.G. Delannoy, S. Greene, A. Gurrola, W. Johns, C. Maguire, Y. Mao, A. Melo, M. Sharma, P. Sheldon, B. Snook, S. Tuo, J. Velkovska

\section{University of Virginia, Charlottesville, USA}

M.W. Arenton, S. Boutle, B. Cox, B. Francis, J. Goodell, R. Hirosky, A. Ledovskoy, H. Li, C. Lin, C. Neu, J. Wood

\section{Wayne State University, Detroit, USA}

C. Clarke, R. Harr, P.E. Karchin, C. Kottachchi Kankanamge Don, P. Lamichhane, J. Sturdy

\section{University of Wisconsin, Madison, USA}

D.A. Belknap, D. Carlsmith, M. Cepeda, S. Dasu, L. Dodd, S. Duric, E. Friis, R. HallWilton, M. Herndon, A. Hervé, P. Klabbers, A. Lanaro, C. Lazaridis, A. Levine, R. Loveless, A. Mohapatra, I. Ojalvo, T. Perry, G.A. Pierro, G. Polese, I. Ross, T. Sarangi, A. Savin, W.H. Smith, D. Taylor, P. Verwilligen, C. Vuosalo, N. Woods

\section{t: Deceased}

1: Also at Vienna University of Technology, Vienna, Austria

2: Also at CERN, European Organization for Nuclear Research, Geneva, Switzerland

3: Also at Institut Pluridisciplinaire Hubert Curien, Université de Strasbourg, Université de Haute Alsace Mulhouse, CNRS/IN2P3, Strasbourg, France

4: Also at National Institute of Chemical Physics and Biophysics, Tallinn, Estonia

5: Also at Skobeltsyn Institute of Nuclear Physics, Lomonosov Moscow State University, Moscow, Russia

6: Also at Universidade Estadual de Campinas, Campinas, Brazil

7: Also at Laboratoire Leprince-Ringuet, Ecole Polytechnique, IN2P3-CNRS, Palaiseau, France

8: Also at Joint Institute for Nuclear Research, Dubna, Russia

9: Also at Suez University, Suez, Egypt

10: Also at Cairo University, Cairo, Egypt

11: Also at Fayoum University, El-Fayoum, Egypt

12: Also at British University in Egypt, Cairo, Egypt

13: Now at Ain Shams University, Cairo, Egypt 
14: Also at Université de Haute Alsace, Mulhouse, France

15: Also at Brandenburg University of Technology, Cottbus, Germany

16: Also at Institute of Nuclear Research ATOMKI, Debrecen, Hungary

17: Also at Eötvös Loránd University, Budapest, Hungary

18: Also at University of Debrecen, Debrecen, Hungary

19: Also at University of Visva-Bharati, Santiniketan, India

20: Now at King Abdulaziz University, Jeddah, Saudi Arabia

21: Also at University of Ruhuna, Matara, Sri Lanka

22: Also at Isfahan University of Technology, Isfahan, Iran

23: Also at Sharif University of Technology, Tehran, Iran

24: Also at Plasma Physics Research Center, Science and Research Branch, Islamic Azad University, Tehran, Iran

25: Also at Università degli Studi di Siena, Siena, Italy

26: Also at Centre National de la Recherche Scientifique (CNRS) - IN2P3, Paris, France

27: Also at Purdue University, West Lafayette, USA

28: Also at Universidad Michoacana de San Nicolas de Hidalgo, Morelia, Mexico

29: Also at Institute for Nuclear Research, Moscow, Russia

30: Also at St. Petersburg State Polytechnical University, St. Petersburg, Russia

31: Also at California Institute of Technology, Pasadena, USA

32: Also at Faculty of Physics, University of Belgrade, Belgrade, Serbia

33: Also at Facoltà Ingegneria, Università di Roma, Roma, Italy

34: Also at Scuola Normale e Sezione dell'INFN, Pisa, Italy

35: Also at University of Athens, Athens, Greece

36: Also at Paul Scherrer Institut, Villigen, Switzerland

37: Also at Institute for Theoretical and Experimental Physics, Moscow, Russia

38: Also at Albert Einstein Center for Fundamental Physics, Bern, Switzerland

39: Also at Gaziosmanpasa University, Tokat, Turkey

40: Also at Adiyaman University, Adiyaman, Turkey

41: Also at Cag University, Mersin, Turkey

42: Also at Anadolu University, Eskisehir, Turkey

43: Also at Izmir Institute of Technology, Izmir, Turkey

44: Also at Necmettin Erbakan University, Konya, Turkey

45: Also at Ozyegin University, Istanbul, Turkey

46: Also at Marmara University, Istanbul, Turkey

47: Also at Kafkas University, Kars, Turkey

48: Also at Rutherford Appleton Laboratory, Didcot, UK

49: Also at School of Physics and Astronomy, University of Southampton, Southampton, UK

50: Also at University of Belgrade, Faculty of Physics and Vinca Institute of Nuclear Sciences, Belgrade, Serbia

51: Also at Mimar Sinan University, Istanbul, Istanbul, Turkey

52: Also at Argonne National Laboratory, Argonne, USA

53: Also at Erzincan University, Erzincan, Turkey

54: Also at Yildiz Technical University, Istanbul, Turkey

55: Also at Texas A\&M University at Qatar, Doha, Qatar

56: Also at Kyungpook National University, Daegu, Korea 